\title{
WAVE PROPAGATION IN SHALLOW-WATER ACOUSTIC RANDOM WAVEGUIDES*
}

\author{
CHRISTOPHE GOMEZ
}

\begin{abstract}
In shallow-water waveguides a propagating field can be decomposed in three kinds of modes: the propagating modes, the radiating modes and the evanescent modes. In this paper we consider the propagation of a wave in a randomly perturbed waveguide and we analyze the coupling between these three kinds of modes using an asymptotic analysis based on a separation of scales technique. Then, we derive the asymptotic form of the distribution of the mode amplitudes and the coupled power equation for propagating modes. From this equation, we show that the total energy carried by the propagating modes decreases exponentially with the size of the random section and we give an expression of the decay rate. Moreover, we show that the mean propagating mode powers converge to the solution of a diffusion equation in the limit of a large number of propagating modes.
\end{abstract}

Key words. Acoustic waveguides, random media, asymptotic analysis.

AMS subject classifications. 76B15, 35Q99, 60F05.

\section{Introduction}

Acoustic wave propagation in shallow-water waveguides has been studied for a long time because of its numerous domains of applications. One of the most important applications is submarine detection with active or passive sonars, but it can also be used in underwater communication, mines or archaeological artifacts detection, and to study the ocean's structure or ocean biology. Shallow-waters are complicated media because they have indices of refraction with spatial and time dependences. However, the sound speed in water, which is about $1500 \mathrm{~m} / \mathrm{s}$, is sufficiently large with respect to the motions of water masses to consider this medium as being time independent. Moreover, the presence of spatial inhomogeneities in the water produces a mode coupling which can induce significant effects over large propagation distances.

In shallow-water waveguides the transverse section can be represented as a semiinfinite interval (see Figure 1.1) and then a wave field can be decomposed into three kinds of modes: the propagating modes which propagate over long distances, the evanescent modes which decrease exponentially with the propagation distance, and the radiating modes representing modes which penetrate under the bottom of the water. The main purpose of this paper is to analyze how the propagating mode powers are affected by the radiating and evanescent modes. This analysis is carried out using an asymptotic analysis based on a separation of scale technique, where the wavelength and the correlation lengths of the inhomogeneities, which are of the same order, are small compared to the propagation distance. Moreover, the relative fluctuations of the medium parameters are small on the scale of the square root of the wave length over the propagation distance. This is the interesting scaling regime corresponding to propagating modes and where the coupling via the environment gives a strong mode coupling. In the terminology of [7] this is the so-called weakly heterogeneous regime.

Wave propagation in random waveguides with a bounded cross-section and Dirichlet boundary conditions (see Figure 1.1) has been studied, for instance, in [7, chapter

\footnotetext{
* Received: December 4, 2009; accepted (in revised version): May 14, 2010. Communicated by Lenya Ryzhik.

†Laboratoire de Probabilités et Modèles Aléatoires, Université Paris 7, Boite courrier 7012, 75251 PARIS Cedex 05, FRANCE (chgomez@math.jussieu.fr).
} 
20] or [9]. In this case we have only two kinds of modes: the propagating and the evanescent modes. In such a model an asymptotic analysis of the mode powers shows total energy conservation and an equipartition of the energy carried by the propagating modes. In [9] coupled power equations are derived under the assumption that evanescent modes are negligible. In [8] the role of evanescent modes is studied in the absence of radiating modes. In this paper we take into account the influence of the radiating and the evanescent modes on the coupled power equations. In this case we show a mode-dependent and frequency-dependent attenuation on the propagating modes in Theorem 6.1, that is, the total energy carried by the propagating modes decreases exponentially with the size of the random section and we give an expression of the decay rate. Moreover, in the limit of a large number of propagating modes $N(\omega) \gg 1$, we show in Theorems 6.2 and 6.5 that the propagating mode powers converge to the solution of a diffusion equation. All the results of this paper are also valid for electromagnetic wave propagation in dielectric waveguides and optical fibers $[19,20,26,27,31]$.

The organization of this paper is as follows: in Section 2 we present the waveguide model, and in Section 3 we present the mode decomposition for a monochromatic wave associated to that model and studied in detail in [30]. In Section 4 we study the mode coupling when the three kinds of modes are taken into account. In the same spirit as in [7, Chapter 20], we derive the coupled mode equations, and we study the energy flux for the propagating and the radiating modes and the influence of the evanescent modes on the two other kinds of modes. In Section 5, under the forward scattering approximation, we study the asymptotic form of the joint distribution of the propagating and radiating mode amplitudes. We apply this result in Section 6 to derive the coupled power equations for the propagating modes, which were already obtained in [15] or [20] for instance. In this section we study the influence of the radiating and evanescent modes on the mean propagating mode powers. We show that the total energy carried by the propagating modes decreases exponentially with the size of the random section and we give an expression of the decay rate. In other words, the radiating modes induce a mode-dependent and frequency-dependent attenuation on the propagating modes, which is why these modes are sometimes called dissipative modes. Finally, under the assumption that nearest-neighbor coupling is the main power transfer mechanism, we derive the continuum approximation to the coupled power equation. More precisely, we show that the mean propagating mode powers converge to the solution of a diffusion equation in the limit of a large number of propagating modes. We can refer to $[15,20]$ for further references and discussions about diffusion models. In that regime, we can also observe the exponential decay behavior caused by the radiative loss.

\section{Waveguide model}

We consider a two-dimensional linear acoustic wave model. The conservation equations of mass and linear momentum are given by

$$
\begin{array}{r}
\rho(x, z) \frac{\partial \mathbf{u}}{\partial t}+\nabla p=\mathbf{F}, \\
\frac{1}{K(x, z)} \frac{\partial p}{\partial t}+\nabla \cdot \mathbf{u}=0
\end{array}
$$

where $p$ is the acoustic pressure, $\mathbf{u}$ is the acoustic velocity, $\rho$ is the density of the medium, $K$ is the bulk modulus, and the source is modeled by the forcing term $\mathbf{F}(t, x, z)$. The third coordinate $z$ represents the propagation axis along the waveguide. 
$(a)$

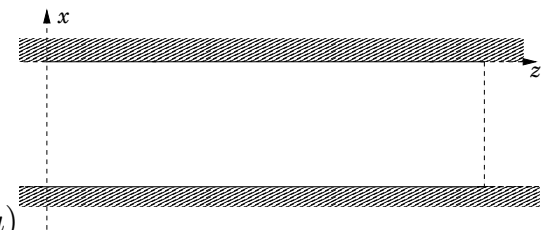

(b) $x_{x}$

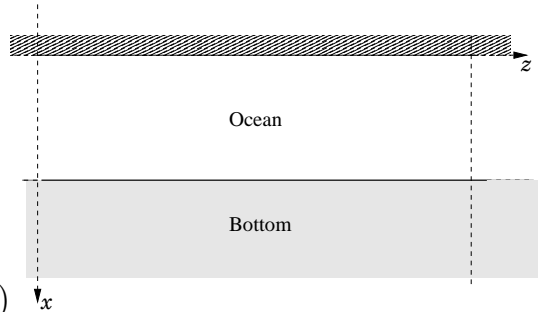

FIG. 1.1. Illustration of two kinds of waveguides. In $(a)$ we show a shallow-water waveguide model with an unbounded cross-section. In (b) we show a waveguide with a bounded cross-section.

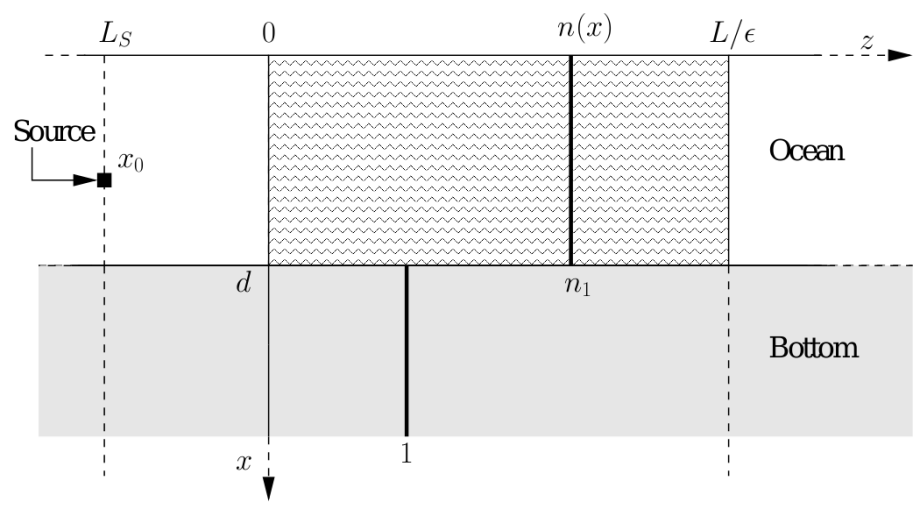

FIG. 2.1. Illustration of the shallow-water waveguide model.

The transverse section of the waveguide is the semi-infinite interval $[0,+\infty)$, and $x \in[0,+\infty)$ represents the transverse coordinate. Let $d>0$, and assume that the medium parameters are given by

$$
\begin{aligned}
\frac{1}{K(x, z)}=\left\{\begin{array}{cl}
\bar{K}^{-1}\left(n^{2}(x)+\sqrt{\epsilon} V(x, z)\right), & \text { if } x \in[0, d], \quad z \in[0, L / \epsilon], \\
\bar{K}^{-1} n^{2}(x), & \text { if }\left\{\begin{array}{l}
x \in[0,+\infty), z \in(-\infty, 0) \cup(L / \epsilon,+\infty) \\
\text { or } \\
x \in(d,+\infty), z \in(-\infty,+\infty) .
\end{array}\right.
\end{array}\right. \\
\rho(x, z)=\bar{\rho} \quad \text { if } \quad x \in[0,+\infty), \quad z \in \mathbb{R} .
\end{aligned}
$$

In this paper we consider the Pekeris waveguide model. This kind of model has been studied for half a century [25], and in this model the index of refraction $n(x)$ is given by

$$
n(x)= \begin{cases}n_{1}>1, & \text { if } x \in[0, d), \\ 1, & \text { if } x \in[d,+\infty)\end{cases}
$$

This profile can model an ocean with a constant sound speed. Such conditions can be found during the winter in Earth's mid latitudes and in water shallower than about 30 meters $[17,13]$. The Pekeris profile leads us to simplified algebra but it underestimates the complexity of the medium. However, the analysis that we present in this paper can be extended to more general profiles $n(x)$ with general boundary 
conditions. In the Pekeris model that we consider $n_{1}$ represents the index of refraction of the ocean section $[0, d]$, where $d$ is the depth of the ocean, and we assume that the index of refraction of the bottom of the ocean is equal to 1 . This model can also be used to study the propagation of electromagnetic waves in a dielectric slab or an optical fiber with randomly perturbed index of refraction [19, 20, 27, 31].

We consider a source pointing in the $z$-direction, which is localized in the plane $z=L_{S}$ :

$$
\mathbf{F}(t, x, z)=\Psi(t, x) \delta\left(z-L_{S}\right) \mathbf{e}_{z} .
$$

$\Psi(t, x)$ represents the profile of the source and $\mathbf{e}_{z}$ is the unit vector pointing in the $z$-direction. $L_{S}<0$ is the location of the source on the propagating axis.

The random process $(V(x, z), x \in[0, d], z \geq 0)$, which models the spatial inhomogeneities, is a continuous real-valued zero-mean stationary Gaussian field with a covariance function given by

$$
\mathbb{E}\left[V\left(x, z_{1}\right) V\left(y, z_{2}\right)\right]=\gamma_{0}(x, y) e^{-a\left|z_{1}-z_{2}\right|} .
$$

Here $a>0$, and $1 / a$ is the longitudinal correlation length of the random fluctuation. Moreover, $\gamma_{0}:[0, d] \times[0, d] \rightarrow \mathbb{R}$ is a continuous function which is the kernel of a nonnegative operator. Using standard properties of Gaussian processes, we can state the following results $[1,2]$. Let

$$
\mathcal{F}_{z}=\sigma(V(x, s), x \in[0, d], s \leq z)
$$

be the $\sigma$-algebra generated by $(V(x, s), x \in[0, d], s \leq z)$. We have the Markov property

$$
\left(V(x, z+h), x \in[0, d] \mid \mathcal{F}_{z}\right)=(V(x, z+h), x \in[0, d] \mid \sigma(V(x, z), x \in[0, d])),
$$

where the equality holds in law, and this law is the one of a Gaussian field with mean

$$
\mathbb{E}\left[V(x, z+h) \mid \mathcal{F}_{z}\right]=e^{-a h} V(x, z)
$$

and covariance $\gamma_{0}(x, y)\left(1-e^{-2 a h}\right)$. Moreover, we shall use the following two properties [2]. First, $\forall T>0, \forall K>0$, and $\forall \mu>0$,

$$
\lim _{\epsilon \rightarrow 0} \mathbb{P}\left(\epsilon^{\mu} \sup _{z \in[0, T]} \sup _{x \in[0, d]}\left|V\left(x, \frac{z}{\epsilon}\right)\right| \geq K\right)=0 .
$$

Second, $\forall n \in \mathbb{N}^{*}$ and $\forall z \geq 0$,

$$
\mathbb{E}\left[\sup _{x \in[0, d]}\left|V\left(x, \frac{z}{\epsilon}\right)\right|^{n}\right]=\mathbb{E}\left[\sup _{x \in[0, d]}|V(x, 0)|^{n}\right]<+\infty .
$$

However, one can remark that the process $V$ is unbounded. This fact implies that the bulk modulus can take negative values. In order to avoid this situation, we can work on the event

$$
\left(\forall(x, z) \in[0, d] \times[0, L / \epsilon], n_{1}+\sqrt{\epsilon} V(x, z)>0\right) .
$$

In fact, the property (2.5) implies

$$
\begin{aligned}
& \lim _{\epsilon \rightarrow 0} \mathbb{P}\left(\exists(x, z) \in[0, d] \times[0, L / \epsilon]: n_{1}+\sqrt{\epsilon} V(x, z) \leq 0\right) \\
\leq & \lim _{\epsilon \rightarrow 0} \mathbb{P}\left(\sqrt{\epsilon} \sup _{z \in[0, L]} \sup _{x \in[0, d]}\left|V\left(x, \frac{z}{\epsilon}\right)\right| \geq n_{1}\right)=0 .
\end{aligned}
$$




\section{Wave propagation in a homogeneous waveguide}

In this section, we assume that the medium parameters are given by

$$
\rho(x, z)=\bar{\rho} \text { and } K(x, z)=\frac{\bar{K}}{n^{2}(x)}, \quad \forall(x, z) \in[0,+\infty) \times \mathbb{R} .
$$

From the conservation equations (2.1), we can derive the wave equation for the pressure field,

$$
\Delta p-\frac{1}{c(x)^{2}} \frac{\partial^{2} p}{\partial t^{2}}=\nabla . \mathbf{F},
$$

where $c(x)=c / n(x)$ with $c=\sqrt{\frac{\bar{K}}{\bar{\rho}}}$, and $\Delta=\partial_{x}^{2}+\partial_{z}^{2}$.

In underwater acoustics the density of air is very small compared to the density of water, and it is natural to use a pressure-release condition. The pressure is very weak outside the waveguide, and by continuity the pressure is zero at the free surface $x=0$. This consideration leads us to consider the Dirichlet boundary conditions

$$
p(t, 0, z)=0 \quad \forall(t, z) \in[0,+\infty) \times \mathbb{R} .
$$

Throughout this paper we consider linear models of propagation. Therefore, the pressure $p(t, x, z)$ can be expressed as the superposition of monochromatic waves by taking its Fourier transform. Here, the Fourier transform and the inverse Fourier transform, with respect to time, are defined by

$$
\widehat{f}(\omega)=\int f(t) e^{i \omega t} d t, \quad f(t)=\frac{1}{2 \pi} \int \widehat{f}(\omega) e^{-i \omega t} d \omega .
$$

In the half-space $z>L_{S}$ (resp., $z<L_{S}$ ), taking the Fourier transform in (3.1), we see that $\widehat{p}(\omega, x, z)$ satisfies the time-harmonic wave equation without source term,

$$
\partial_{z}^{2} \widehat{p}(\omega, x, z)+\partial_{x}^{2} \widehat{p}(\omega, x, z)+k^{2}(\omega) n^{2}(x) \widehat{p}(\omega, x, z)=0,
$$

where $k(\omega)=\frac{\omega}{c}$ is the wavenumber, and with Dirichlet boundary conditions $\widehat{p}(\omega, 0, z)=$ $0 \forall z$. The source term implies the following jump conditions for the pressure field across the plane $z=L_{S}$ :

$$
\begin{aligned}
\widehat{p}\left(\omega, x, L_{S}^{+}\right)-\widehat{p}\left(\omega, x, L_{S}^{-}\right) & =\widehat{\Psi}(\omega, x), \\
\partial_{z} \widehat{p}\left(\omega, x, L_{S}^{+}\right)-\partial_{z} \widehat{p}\left(\omega, x, L_{S}^{-}\right) & =0 .
\end{aligned}
$$

3.1. Spectral decomposition in unperturbed waveguides. This section is devoted to the presentation of the spectral decomposition of the Pekeris operator $\partial_{x}^{2}+k^{2}(\omega) n^{2}(x)$. The spectral analysis of this operator is carried out in [30]. In this paper, we are interested in solutions of (3.2) such that

$$
\begin{aligned}
& \widehat{p}(\omega, ., .) \mathbf{1}_{\left(L_{S},+\infty\right)}(z) \in \mathcal{C}^{0}\left(\left(L_{S},+\infty\right), H_{0}^{1}(0,+\infty) \cap H^{2}(0,+\infty)\right) \cap \mathcal{C}^{2}\left(\left(L_{S},+\infty\right), H\right), \\
& \widehat{p}(\omega, ., .) \mathbf{1}_{\left(-\infty, L_{S}\right)}(z) \in \mathcal{C}^{0}\left(\left(-\infty, L_{S}\right), H_{0}^{1}(0,+\infty) \cap H^{2}(0,+\infty)\right) \cap \mathcal{C}^{2}\left(\left(-\infty, L_{S}\right), H\right),
\end{aligned}
$$

where $H=L^{2}(0,+\infty)$. $H$ is equipped with the inner product defined by

$$
\forall\left(h_{1}, h_{2}\right) \in H \times H, \quad\left\langle h_{1}, h_{2}\right\rangle_{H}=\int_{0}^{+\infty} h_{1}(x) \overline{h_{2}(x)} d x .
$$


Consequently, in the half-space $z>L_{S}$ (resp., $z<L_{S}$ ) we can consider (3.2) as the operator-differential equation

$$
\frac{d^{2}}{d z^{2}} \widehat{p}(\omega, ., z)+R(\omega)(\widehat{p}(\omega, ., z))=0
$$

in $H$, where $R(\omega)$ is an unbounded operator on $H$ with domain

$$
\mathcal{D}(R(\omega))=H_{0}^{1}(0,+\infty) \cap H^{2}(0,+\infty)
$$

and defined by

$$
R(\omega)(y)=\frac{d^{2}}{d x^{2}} y+k^{2}(\omega) n^{2}(x) y, \quad \forall y \in \mathcal{D}(R(\omega)) .
$$

According to [30], $R(\omega)$ is a self-adjoint operator on the Hilbert space $H$, and its spectrum is given by

$$
S p(R(\omega))=\left(-\infty, k^{2}(\omega)\right] \cup\left\{\beta_{N(\omega)}^{2}(\omega), \ldots, \beta_{1}^{2}(\omega)\right\} .
$$

More precisely, $\forall j \in\{1, \ldots, N(\omega)\}$, the modal wavenumber $\beta_{j}(\omega)$ is positive and

$$
k^{2}(\omega)<\beta_{N(\omega)}^{2}(\omega)<\cdots<\beta_{1}^{2}(\omega)<n_{1}^{2} k^{2}(\omega)
$$

Moreover, there exists a resolution of the identity $\Pi_{\omega}$ of $R(\omega)$ such that $\forall y \in H$ and $\forall r \in \mathbb{R}$

$$
\begin{aligned}
\Pi_{\omega}(r,+\infty)(y)(x)= & \sum_{j=1}^{N(\omega)}\left\langle y, \phi_{j}(\omega, .)\right\rangle_{H} \phi_{j}(\omega, x) \mathbf{1}_{(r,+\infty)}\left(\beta_{j}(\omega)^{2}\right) \\
& +\int_{r}^{k^{2}(\omega)}\left\langle y, \phi_{\gamma}(\omega, .)\right\rangle_{H} \phi_{\gamma}(\omega, x) d \gamma \mathbf{1}_{\left(-\infty, k^{2}(\omega)\right)}(r),
\end{aligned}
$$

and $\forall y \in \mathcal{D}(R(\omega))$ and $\forall r \in \mathbb{R}$,

$$
\begin{aligned}
\Pi_{\omega}(r,+\infty)(R(\omega)(y))(x)= & \sum_{j=1}^{N(\omega)} \beta_{j}(\omega)^{2}\left\langle y, \phi_{j}(\omega, .)\right\rangle_{H} \phi_{j}(\omega, x) \mathbf{1}_{(r,+\infty)}\left(\beta_{j}(\omega)^{2}\right) \\
& +\int_{r}^{k^{2}(\omega)} \gamma\left\langle y, \phi_{\gamma}(\omega, x)\right\rangle_{H} \phi_{\gamma}(\omega, x) d \gamma \mathbf{1}_{\left(-\infty, k^{2}(\omega)\right)}(r) .
\end{aligned}
$$

Let us describe these decompositions.

Discrete part of the decomposition

$\forall j \in\{1, \ldots, N(\omega)\}$, the $j$ th eigenvector is given by [30]

$$
\phi_{j}(\omega, x)=\left\{\begin{array}{cl}
A_{j}(\omega) \sin \left(\sigma_{j}(\omega) x / d\right), & \text { if } 0 \leq x \leq d, \\
A_{j}(\omega) \sin \left(\sigma_{j}(\omega)\right) e^{-\zeta_{j}(\omega) \frac{x-d}{d},}, & \text { if } d \leq x,
\end{array}\right.
$$

where

$$
\sigma_{j}(\omega)=d \sqrt{n_{1}^{2} k^{2}(\omega)-\beta_{j}^{2}(\omega)}, \quad \zeta_{j}(\omega)=d \sqrt{\beta_{j}^{2}(\omega)-k^{2}(\omega)},
$$


and

$$
A_{j}(\omega)=\sqrt{\frac{2 / d}{1+\frac{\sin ^{2}\left(\sigma_{j}(\omega)\right)}{\zeta_{j}(\omega)}-\frac{\sin \left(2 \sigma_{j}(\omega)\right)}{2 \sigma_{j}(\omega)}}} .
$$

According to [30], $\sigma_{1}(\omega), \ldots, \sigma_{N(\omega)}(\omega)$ are the solutions on $\left(0, n_{1} k(\omega) d \theta\right)$ of the equation

$$
\tan (y)=-\frac{y}{\sqrt{\left(n_{1} k d \theta\right)^{2}-y^{2}}},
$$

such that $0<\sigma_{1}(\omega)<\cdots<\sigma_{N(\omega)}(\omega)<n_{1} k(\omega) d \theta$, and with $\theta=\sqrt{1-1 / n_{1}^{2}}$. This last equation admits exactly one solution over each interval of the form $(\pi / 2+(j-$ 1) $\pi, \pi / 2+j \pi)$ for $j \in\{1, \ldots, N(\omega)\}$, where

$$
N(\omega)=\left[\frac{n_{1} k(\omega) d}{\pi} \theta\right]
$$

and [.] stands for the integer part. From (3.8) we get the following results which are used to show the main result of Section 6.2.

Lemma 3.1. Let $\alpha>1 / 3$. We have as $N(\omega) \rightarrow+\infty$

$$
\begin{gathered}
\sup _{j \in\left\{1, \ldots, N(\omega)-\left[N(\omega)^{\alpha}\right]-1\right\}}\left|\sigma_{j+1}(\omega)-\sigma_{j}(\omega)-\pi\right|=\mathcal{O}\left(N(\omega)^{\frac{1}{2}-\frac{3}{2} \alpha}\right) . \\
\sup _{j \in\left\{1, \ldots, N(\omega)-\left[N(\omega)^{\alpha}\right]-2\right\}} \mid \sigma_{j+2}(\omega)-2 \sigma_{j+1}(\omega)+\sigma_{j}(\omega) \text { right } \mid=\mathcal{O}\left(N(\omega)^{1-3 \alpha}\right) .
\end{gathered}
$$

Let us note that, $\forall \eta \in[0,1[$, we have

$$
\sup _{j \in\left\{1, \ldots, N(\omega)^{\eta}\right\}}\left|\sigma_{j}(\omega)-j \pi\right|=\mathcal{O}\left(N(\omega)^{\eta-1}\right),
$$

and

$$
\lim _{N(\omega) \rightarrow+\infty} \sup _{j \in\left\{1, \ldots, N(\omega)^{\eta}\right\}}\left\|\phi_{j}(\omega, .)-\phi_{j}(\infty, .)\right\|_{H}=0
$$

with

$$
\phi_{j}(\infty, x)=\left\{\begin{array}{cl}
\sqrt{\frac{2}{d}} \sin \left(j \frac{\pi}{d} x\right), & \text { if } x \in[0, d], \\
0, & \text { if } x \geq d .
\end{array}\right.
$$

This result means that in the limit of a large number of propagating modes the low order propagating modes are very similar in shape to those of a perfect bounded waveguide with pressure-release boundary conditions at $x=0$ and $x=d$ (see Figure 1.1 (b)). Indeed, according to (3.6), in the limit of a large number of propagating modes, the low order propagating modes have a small amplitude near the bottom. This approximation does not hold anymore for high order propagating modes. However, the results of Lemma 3.1 mean that for the high order propagating modes the distribution of solutions of (3.8) approaches the distribution of the eigenvalues of the transverse Laplacian associated to a perfect bounded waveguide with pressure-release boundary conditions at $x=0$ and $x=1$. 
Continuous part of the decomposition

For $\gamma \in\left(-\infty, k^{2}(\omega)\right)$, we have [30]

$$
\begin{aligned}
& \phi_{\gamma}(\omega, x)= \\
& \left\{\begin{array}{cl}
A_{\gamma}(\omega) \sin (\eta(\omega) x / d), & \text { if } 0 \leq x \leq d, \\
A_{\gamma}(\omega)\left(\sin (\eta(\omega)) \cos \left(\xi(\omega) \frac{x-d}{d}\right)+\frac{\eta(\omega)}{\xi(\omega)} \cos (\eta(\omega)) \sin \left(\xi(\omega) \frac{x-d}{d}\right)\right), & \text { if } d \leq x,
\end{array}\right.
\end{aligned}
$$

where

$$
\eta(\omega)=d \sqrt{n_{1}^{2} k^{2}(\omega)-\gamma}, \quad \xi(\omega)=d \sqrt{k^{2}(\omega)-\gamma}
$$

and

$$
A_{\gamma}(\omega)=\sqrt{\frac{d \xi(\omega)}{\pi\left(\xi^{2}(\omega) \sin ^{2}(\eta(\omega))+\eta^{2}(\omega) \cos ^{2}(\eta(\omega))\right)}}
$$

It is easy to check that the function $\gamma \mapsto A_{\gamma}(\omega)$ is continuous on $\left(-\infty, k^{2}(\omega)\right)$ and

$$
A_{\gamma}(\omega) \underset{\gamma \rightarrow-\infty}{\sim} \frac{1}{\sqrt{\pi}|\gamma|^{1 / 4}} .
$$

We remark that $\phi_{\gamma}(\omega,$.$) does not belong to H$. Then $\left\langle y, \phi_{\gamma}(\omega, .)\right\rangle_{H}$ is not defined in the classical way. In fact, we have

$$
\left\langle y, \phi_{\gamma}(\omega, .)\right\rangle_{H}=\lim _{M \rightarrow+\infty} \int_{0}^{M} y(x) \phi_{\gamma}(\omega, x) d x
$$

where the limit holds on $L^{2}\left(-\infty, k^{2}(\omega)\right)$. Moreover, we have, $\forall y \in H$,

$$
\|y\|_{H}^{2}=\left\|\Pi_{\omega}(-\infty,+\infty)(y)\right\|_{H}^{2}=\sum_{j=1}^{N(\omega)}\left|\left\langle y, \phi_{j}(\omega, .)\right\rangle_{H}\right|^{2}+\int_{-\infty}^{k^{2}(\omega)}\left|\left\langle y, \phi_{\gamma}(\omega, .)\right\rangle_{H}\right|^{2} d \gamma
$$

and therefore the map which assigns to every element of $H$ the coefficients of its spectral decomposition

$$
\begin{aligned}
\Theta_{\omega}: H & \longrightarrow \mathcal{H}^{\omega} \\
y & \longrightarrow\left(\left(\left\langle y, \phi_{j}(\omega, .)\right\rangle_{H}\right)_{j=1, \ldots, N(\omega)},\left(\left\langle y, \phi_{\gamma}(\omega, .)\right\rangle_{H}\right)_{\gamma \in\left(-\infty, k^{2}(\omega)\right)}\right)
\end{aligned}
$$

is an isometry from $H$ onto $\mathcal{H}^{\omega}=\mathbb{C}^{N(\omega)} \times L^{2}\left(-\infty, k^{2}(\omega)\right)$.

3.2. Modal decomposition. In this section we apply the spectral decomposition introduced in Section 3.1 on a solution $\widehat{p}(\omega, x, z)$ of the equation (3.4). Consequently, we get the modal decomposition for $\widehat{p}(\omega, x, z)$ in the half-space $z>L_{S}$,

$\widehat{p}(\omega, x, z)=\sum_{j=1}^{N(\omega)} \widehat{p}_{j}(\omega, z) \phi_{j}(\omega, x)+\int_{0}^{k^{2}(\omega)} \widehat{p}_{\gamma}(\omega, z) \phi_{\gamma}(\omega, x) d \gamma+\int_{-\infty}^{0} \widehat{p}_{\gamma}(\omega, z) \phi_{\gamma}(\omega, x) d \gamma$

where

$$
\begin{aligned}
\widehat{p}_{j}(\omega, z) & =\Theta_{\omega} \circ \Pi_{\omega}(\{j\})(\widehat{p}(\omega, ., z)) \quad \text { for } j \in\{1, \ldots, N(\omega)\}, \\
\left(\widehat{p}_{\gamma}(\omega, z)\right)_{\gamma \in\left(0, k^{2}(\omega)\right)} & =\Theta_{\omega} \circ \Pi_{\omega}\left(0, k^{2}(\omega)\right)(\widehat{p}(\omega, ., z)), \quad \text { and } \\
\left(\widehat{p}_{\gamma}(\omega, z)\right)_{\gamma \in(-\infty, 0)} & =\Theta_{\omega} \circ \Pi_{\omega}(-\infty, 0)(\widehat{p}(\omega, ., z)) .
\end{aligned}
$$


For $j \in\{1, \ldots, N(\omega)\}, \Theta_{\omega} \circ \Pi_{\omega}(\{j\})$ represents the projection onto the $j$ th propagating mode, and $\widehat{p}_{j}(\omega, z)$ is the amplitude of the $j$ th propagating mode. $\Theta_{\omega} \circ \Pi_{\omega}\left(0, k^{2}(\omega)\right)$ represents the projection onto the radiating modes, and $\widehat{p}_{\gamma}(\omega, z)$ is the amplitude of the $\gamma$ th radiating mode for almost every $\gamma \in\left(0, k^{2}(\omega)\right)$. Finally, $\Theta_{\omega} \circ \Pi_{\omega}(-\infty, 0)$ represents the projection onto the evanescent modes and $\widehat{p}_{\gamma}(\omega, z)$ is the amplitude of the $\gamma$ th evanescent mode for almost every $\gamma \in(-\infty, 0)$.

Consequently, $\widehat{p}(\omega, z)$ satisfies

$$
\begin{aligned}
\frac{d^{2}}{d z^{2}} \widehat{p}_{j}(\omega, z)+\beta_{j}^{2}(\omega) \widehat{p}_{j}(\omega, z) & =0, \\
\frac{d^{2}}{d z^{2}} \widehat{p}_{\gamma}(\omega, z)+\gamma \widehat{p}_{\gamma}(\omega, z) & =0
\end{aligned}
$$

in $\mathcal{H}^{\omega}$. Therefore, the pressure field can be written as an expansion over the complete set of modes

$$
\begin{gathered}
\widehat{p}(\omega, x, z)=\left[\sum_{j=1}^{N(\omega)} \frac{\widehat{a}_{j, 0}(\omega)}{\sqrt{\beta_{j}(\omega)}} e^{i \beta_{j}(\omega) z} \phi_{j}(\omega, x)+\int_{0}^{k^{2}(\omega)} \frac{\widehat{a}_{\gamma, 0}(\omega)}{\gamma^{1 / 4}} e^{i \sqrt{\gamma} z} \phi_{\gamma}(\omega, x) d \gamma\right. \\
\left.+\int_{-\infty}^{0} \frac{\widehat{c}_{\gamma, 0}(\omega)}{|\gamma|^{1 / 4}} e^{-\sqrt{|\gamma|} z} \phi_{\gamma}(\omega, x) d \gamma\right] \mathbf{1}_{\left(L_{S},+\infty\right)}(z) \\
+\left[\sum_{j=1}^{N(\omega)} \frac{\widehat{b}_{j, 0}(\omega)}{\sqrt{\beta_{j}(\omega)}} e^{-i \beta_{j}(\omega) z} \phi_{j}(\omega, x)+\int_{0}^{k^{2}(\omega)} \frac{\widehat{b}_{\gamma, 0}(\omega)}{\gamma^{1 / 4}} e^{-i \sqrt{\gamma} z} \phi_{\gamma}(\omega, x) d \gamma\right. \\
\left.+\int_{-\infty}^{0} \frac{\widehat{d}_{\gamma, 0}(\omega)}{|\gamma|^{\mid / 4}} e^{\sqrt{|\gamma|} z} \phi_{\gamma}(\omega, x) d \gamma\right] \mathbf{1}_{\left(-\infty, L_{S}\right)}(z)
\end{gathered}
$$

under the assumption that $\left(\widehat{c}_{\gamma, 0}(\omega) e^{-\sqrt{|\gamma|} L_{S}} /|\gamma|^{1 / 4}\right)_{\gamma}$ and $\left(\widehat{d}_{\gamma, 0}(\omega) e^{\sqrt{|\gamma|} L_{S}} /|\gamma|^{1 / 4}\right)_{\gamma}$ belong to the space $L^{2}(-\infty, 0)$, and with the radiation condition for the evanescent modes

$$
\lim _{z \rightarrow+\infty}\left\|\Pi_{\omega}(-\infty, 0)(\widehat{p}(\omega, ., z))\right\|_{H}^{2}=0 .
$$

This condition means that the energy carried by the evanescent modes decays as the propagation distance becomes large. In the previous decomposition, $\widehat{a}_{j, 0}(\omega)$ (resp., $\widehat{b}_{j, 0}(\omega)$ ) is the amplitude of the $j$ th right-going (resp., left-going) mode propagating in the right half-space $z>L_{S}$ (resp., left half-space $z<L_{S}$ ), $\widehat{a}_{\gamma, 0}(\omega)$ (resp., $\widehat{b}_{\gamma, 0}(\omega)$ ) is the amplitude of the $\gamma$ th right-going (resp., left-going) mode radiating in the right half-space $z>L_{S}$ (resp., left half-space $z<L_{S}$ ), and $\widehat{c}_{\gamma, 0}(\omega)$ (resp., $\widehat{d}_{\gamma, 0}(\omega)$ ) is the amplitude of the $\gamma$ th right-going (resp., left-going) evanescent mode in the right halfspace $z>L_{S}$ (resp., left half-space $z<L_{S}$ ).

We assume that the profile $\Psi(t, x)$ of the source term (2.3) is given, in the frequency domain, by

$$
\widehat{\Psi}(\omega, x)=\widehat{f}(\omega)\left[\sum_{j=1}^{N(\omega)} \phi_{j}\left(\omega, x_{0}\right) \phi_{j}(\omega, x)+\int_{(-S,-\xi) \cup\left(\xi, k^{2}(\omega)\right)} \phi_{\gamma}\left(\omega, x_{0}\right) \phi_{\gamma}(\omega, x) d \gamma\right],
$$


where $x_{0} \in(0, d)$. The bound $S$ in the spectral decomposition of the source profile was introduced to have $\widehat{\Psi}(\omega,.) \in H$, and $\xi$ was introduced for technical reasons as discussed below. Note that $S$ can be arbitrarily large and $\xi$ can be arbitrarily small. Therefore, the spatial profile in (3.13) is an approximation of a Dirac distribution at $x_{0}$, which models a point source at $x_{0}$.

Applying $\Theta_{\omega}$ on (3.3) and using (3.12), we obtain

$$
\begin{aligned}
& \widehat{a}_{j, 0}(\omega)=-\overline{\widehat{b}_{j, 0}(\omega)}=\frac{\sqrt{\beta_{j}(\omega)}}{2} \widehat{f}(\omega) \phi_{j}\left(\omega, x_{0}\right) e^{-i \beta_{j}(\omega) L_{S}} \quad \forall j \in\{1, \ldots, N(\omega)\}, \\
& \widehat{a}_{\gamma, 0}(\omega)=-\overline{\widehat{b}_{\gamma, 0}(\omega)}=\left\{\begin{array}{cc}
\frac{\gamma^{1 / 4}}{2} \widehat{f}(\omega) \phi_{\gamma}\left(\omega, x_{0}\right) e^{-i \sqrt{\gamma} L_{S}} & \text { for almost every } \gamma \in\left(\xi, k^{2}(\omega)\right), \\
0 & \text { for almost every } \gamma \in(0, \xi),
\end{array}\right. \\
& \widehat{c}_{\gamma, 0}(\omega)=-\frac{\gamma^{1 / 4}}{2} \widehat{f}(\omega) \phi_{\gamma}\left(\omega, x_{0}\right) e^{\sqrt{|\gamma|} L_{S}}, \quad \widehat{d}_{\gamma, 0}(\omega)=\frac{\gamma^{1 / 4}}{2} \widehat{f}(\omega) \phi_{\gamma}\left(\omega, x_{0}\right) e^{-\sqrt{|\gamma|} L_{S}}
\end{aligned}
$$

for almost every $\gamma \in(-S,-\xi)$, and

$$
\widehat{c}_{\gamma, 0}(\omega)=\widehat{d}_{\gamma, 0}(\omega)=0
$$

for almost every $\gamma \in(-\infty,-S) \cup(-\xi, 0)$.

\section{Mode coupling in random waveguides}

In this section we study the expansion of $\widehat{p}(\omega, x, z)$ when a random section $[0, L / \epsilon]$ is inserted between two homogeneous waveguides (see Figure 2.1). In this section the medium parameters are given by

$$
\begin{aligned}
& \frac{1}{K(x, z)}=\left\{\begin{array}{cl}
\bar{K}^{-1}\left(n^{2}(x)+\sqrt{\epsilon} V(x, z)\right), & \text { if } x \in[0, d], \quad z \in[0, L / \epsilon], \\
\bar{K}^{-1} n^{2}(x), & \text { if }\left\{\begin{array}{l}
x \in[0,+\infty), z \in(-\infty, 0) \cup(L / \epsilon,+\infty), \\
\text { or } \\
x \in(d,+\infty), z \in(-\infty,+\infty) .
\end{array}\right.
\end{array}\right. \\
& \rho(x, z)=\bar{\rho} \quad \text { if } \quad x \in[0,+\infty), \quad z \in \mathbb{R},
\end{aligned}
$$

where $n(x)$ is the Pekeris profile defined by $(2.2)$. However, the analysis that we present can be extended to more general profiles $n(x)$.

In the perturbed section, the pressure field can be decomposed using the resolution of the identity $\Pi_{\omega}$ of the unperturbed waveguide:

$$
\widehat{p}(\omega, x, z)=\sum_{j=1}^{N(\omega)} \widehat{p}_{j}(\omega, z) \phi_{j}(\omega, x)+\int_{0}^{k^{2}(\omega)} \widehat{p}_{\gamma}(\omega, z) \phi_{\gamma}(\omega, x) d \gamma+\int_{-\infty}^{0} \widehat{p}_{\gamma}(\omega, z) \phi_{\gamma}(\omega, x) d \gamma
$$

where $\widehat{p}(\omega, z)=\Theta_{\omega}(\widehat{p}(\omega, ., z))$ as in (3.11). In what follows, we consider solutions of the form

$$
\widehat{p}(\omega, x, z)=\sum_{j=1}^{N(\omega)} \widehat{p}_{j}(\omega, z) \phi_{j}(\omega, x)+\int_{(-\infty,-\xi) \cup\left(\xi, k^{2}(\omega)\right)} \widehat{p}_{\gamma}(\omega, z) \phi_{\gamma}(\omega, x) d \gamma .
$$

According to (3.13) this assumption is tantamount to cancel the coupling to an arbitrarily small band of modes $(-\xi, \xi)$ (which are the quasi-stationary modes), that is, $\Pi_{\omega}(-\xi, \xi)(V(., z) \widehat{p}(\omega, ., z))=0$. This assumption leads us to simplified algebra in 
the proof of Theorem 5.4. In such a decomposition, the radiating and the evanescent part are separated by the small band $(-\xi, \xi)$ with $\xi \ll 1$. The goal is to isolate the transition mode 0 between the radiating and the evanescent parts of the spectrum $S p(R(\omega))$ given by (3.5). Moreover, we assume that $\epsilon \ll \xi$ and therefore that we have two distinct scales. In this paper, we shall consider first the asymptotic when $\epsilon$ goes to 0 and then the asymptotic when $\xi$ goes to 0 .

4.1. Coupled mode equations. In this section we give the coupled mode equations, which describe the coupling mechanism between the amplitudes of the three kinds of modes.

In the random section $[0, L / \epsilon]$ the pressure field $\widehat{p}(\omega, z)$ satisfies the following coupled equations in $\mathcal{H}^{\omega}$ :

$$
\begin{aligned}
\frac{d^{2}}{d z^{2}} \widehat{p}_{j}(\omega, z)+\beta_{j}^{2}(\omega) \widehat{p}_{j}(\omega, z) & +\sqrt{\epsilon} k^{2}(\omega) \sum_{l=1}^{N(\omega)} C_{j l}^{\omega}(z) \widehat{p}_{l}(\omega, z) \\
& +\sqrt{\epsilon} k^{2}(\omega) \int_{(-\infty,-\xi) \cup\left(\xi, k^{2}(\omega)\right)} C_{j \gamma^{\prime}}^{\omega}(z) \widehat{p}_{\gamma^{\prime}}(\omega, z) d \gamma^{\prime}=0, \\
\frac{d^{2}}{d z^{2}} \widehat{p}_{\gamma}(\omega, z)+\gamma \widehat{p}_{\gamma}(\omega, z) & +\sqrt{\epsilon} k^{2}(\omega) \sum_{l=1}^{N(\omega)} C_{\gamma l}^{\omega}(z) \widehat{p}_{l}(\omega, z) \\
& +\sqrt{\epsilon} k^{2}(\omega) \int_{(-\infty,-\xi) \cup\left(\xi, k^{2}(\omega)\right)} C_{\gamma \gamma^{\prime}}^{\omega}(z) \widehat{p}_{\gamma^{\prime}}(\omega, z) d \gamma^{\prime}=0,
\end{aligned}
$$

where

$$
\begin{aligned}
C_{j l}^{\omega}(z) & =\left\langle\phi_{j}(\omega, .), \phi_{l}(\omega, .) V(., z)\right\rangle_{H}=\int_{0}^{d} \phi_{j}(\omega, x) \phi_{l}(\omega, x) V(x, z) d x, \\
C_{j \gamma}^{\omega}(z) & =C_{\gamma j}(z)=\left\langle\phi_{j}(\omega, .), \phi_{\gamma}(\omega, .) V(., z)\right\rangle_{H}=\int_{0}^{d} \phi_{j}(\omega, x) \phi_{\gamma}(\omega, x) V(x, z) d x, \\
C_{\gamma \gamma^{\prime}}^{\omega}(z) & =\left\langle\phi_{\gamma}(\omega, .), \phi_{\gamma^{\prime}}(\omega, .) V(., z)\right\rangle_{H}=\int_{0}^{d} \phi_{\gamma}(\omega, x) \phi_{\gamma^{\prime}}(\omega, x) V(x, z) d x .
\end{aligned}
$$

We recall that $\widehat{p}(\omega, . ..) \in \mathcal{C}^{0}\left((0,+\infty), H_{0}^{1}(0,+\infty) \cap H^{2}(0,+\infty)\right) \cap \mathcal{C}^{2}((0,+\infty), H)$,

$$
\int_{-\infty}^{-\xi} \gamma^{2}\left|\widehat{p}_{\gamma}(\omega, z)\right|^{2} d \gamma<+\infty
$$

In the previous coupled equation the coefficients $C^{\omega}(z)$ represent the coupling between the three kinds of modes, which are the propagating, radiating, and evanescent modes.

Next, we introduce the amplitudes of the generalized right- and left-going modes $\widehat{a}(\omega, z)$ and $\widehat{b}(\omega, z)$, which are given by

$$
\begin{aligned}
\widehat{p}_{j}(\omega, z) & =\frac{1}{\sqrt{\beta_{j}(\omega)}}\left(\widehat{a}_{j}(\omega, z) e^{i \beta_{j}(\omega) z}+\widehat{b}_{j}(\omega, z) e^{-i \beta_{j}(\omega) z}\right), \\
\frac{d}{d z} \widehat{p}_{j}(\omega, z) & =i \sqrt{\beta_{j}(\omega)}\left(\widehat{a}_{j}(\omega, z) e^{i \beta_{j}(\omega) z}-\widehat{b}_{j}(\omega, z) e^{-i \beta_{j}(\omega) z}\right), \\
\widehat{p}_{\gamma}(\omega, z) & =\frac{1}{\gamma^{1 / 4}}\left(\widehat{a}_{\gamma}(\omega, z) e^{i \sqrt{\gamma} z}+\widehat{b}_{\gamma}(\omega, z) e^{-i \sqrt{\gamma} z}\right), \\
\frac{d}{d z} \widehat{p}_{\gamma}(\omega, z) & =i \gamma^{1 / 4}\left(\widehat{a}_{\gamma}(\omega, z) e^{i \sqrt{\gamma} z}-\widehat{b}_{\gamma}(\omega, z) e^{-i \sqrt{\gamma} z}\right)
\end{aligned}
$$


$\forall j \in\{1, \ldots, N(\omega)\}$ and almost every $\gamma \in\left(\xi, k^{2}(\omega)\right)$. Let

$$
\mathcal{H}_{\xi}^{\omega}=\mathbb{C}^{N(\omega)} \times L^{2}\left(\xi, k^{2}(\omega)\right)
$$

From (4.1), we obtain the coupled mode equation in $\mathcal{H}_{\xi}^{\omega} \times \mathcal{H}_{\xi}^{\omega} \times L^{2}(-\infty,-\xi)$ for the amplitudes $(\widehat{a}(\omega, z), \widehat{b}(\omega, z), \widehat{p}(\omega, z))$ :

$$
\begin{aligned}
& \frac{d}{d z} \widehat{a}_{j}(\omega, z)=\sqrt{\epsilon} \frac{i k^{2}(\omega)}{2} \sum_{l=1}^{N(\omega)} \frac{C_{j l}^{\omega}(z)}{\sqrt{\beta_{j} \beta_{l}}}\left(\widehat{a}_{l}(\omega, z) e^{i\left(\beta_{l}-\beta_{j}\right) z}+\widehat{b}_{l}(\omega, z) e^{-i\left(\beta_{l}+\beta_{j}\right) z}\right) \\
& +\sqrt{\epsilon} \frac{i k^{2}(\omega)}{2} \int_{\xi}^{k^{2}(\omega)} \frac{C_{j \gamma^{\prime}}^{\omega}(z)}{\sqrt{\beta_{j} \sqrt{\gamma^{\prime}}}}\left(\widehat{a}_{\gamma^{\prime}}(\omega, z) e^{i\left(\sqrt{\gamma^{\prime}}-\beta_{j}\right) z}+\widehat{b}_{\gamma^{\prime}}(\omega, z) e^{-i\left(\sqrt{\gamma^{\prime}}+\beta_{j}\right) z}\right) d \gamma^{\prime} \\
& +\sqrt{\epsilon} \frac{i k^{2}(\omega)}{2} \int_{-\infty}^{-\xi} \frac{C_{j \gamma^{\prime}}^{\omega}(z)}{\sqrt{\beta_{j}}} \widehat{p}_{\gamma^{\prime}}(\omega, z) d \gamma^{\prime} e^{-i \beta_{j} z} \\
& \frac{d}{d z} \widehat{a}_{\gamma}(\omega, z)=\sqrt{\epsilon} \frac{i k^{2}(\omega)}{2} \sum_{l=1}^{N(\omega)} \frac{C_{\gamma l}^{\omega}(z)}{\sqrt{\sqrt{\gamma} \beta_{l}}}\left(\widehat{a}_{l}(\omega, z) e^{i\left(\beta_{l}-\sqrt{\gamma}\right) z}+\widehat{b}_{l}(\omega, z) e^{-i\left(\beta_{l}+\sqrt{\gamma}\right) z}\right) \\
& +\sqrt{\epsilon} \frac{i k^{2}(\omega)}{2} \int_{\xi}^{k^{2}(\omega)} \frac{C_{\gamma \gamma^{\prime}}^{\omega}(z)}{\gamma^{1 / 4} \gamma^{1 / 4}}\left(\widehat{a}_{\gamma^{\prime}}(\omega, z) e^{i\left(\sqrt{\gamma^{\prime}}-\sqrt{\gamma}\right) z}+\widehat{b}_{\gamma^{\prime}}(\omega, z) e^{-i\left(\sqrt{\gamma^{\prime}}+\sqrt{\gamma}\right) z}\right) d \gamma^{\prime} \\
& +\sqrt{\epsilon} \frac{i k^{2}(\omega)}{2} \int_{-\infty}^{-\xi} \frac{C_{\gamma \gamma^{\prime}}^{\omega}(z)}{\gamma^{1 / 4}} \widehat{p}_{\gamma^{\prime}}(\omega, z) d \gamma^{\prime} e^{-i \gamma z}, \\
& \frac{d}{d z} \widehat{b}_{j}(\omega, z)=-\sqrt{\epsilon} \frac{i k^{2}(\omega)}{2} \sum_{l=1}^{N(\omega)} \frac{C_{j l}^{\omega}(z)}{\sqrt{\beta_{j} \beta_{l}}}\left(\widehat{a}_{l}(\omega, z) e^{i\left(\beta_{l}+\beta_{j}\right) z}+\widehat{b}_{l}(\omega, z) e^{-i\left(\beta_{l}-\beta_{j}\right) z}\right) \\
& -\sqrt{\epsilon} \frac{i k^{2}(\omega)}{2} \int_{\xi}^{k^{2}(\omega)} \frac{C_{j \gamma^{\prime}}^{\omega}(z)}{\sqrt{\beta_{j} \sqrt{\gamma^{\prime}}}}\left(\widehat{a}_{\gamma^{\prime}}(\omega, z) e^{i\left(\sqrt{\gamma^{\prime}}+\beta_{j}\right) z}+\widehat{b}_{\gamma^{\prime}}(\omega, z) e^{-i\left(\sqrt{\gamma^{\prime}}-\beta_{j}\right) z}\right) d \gamma^{\prime} \\
& -\sqrt{\epsilon} \frac{i k^{2}(\omega)}{2} \int_{-\infty}^{-\xi} \frac{C_{j \gamma^{\prime}}^{\omega}(z)}{\sqrt{\beta_{j}}} \widehat{p}_{\gamma^{\prime}}(\omega, z) d \gamma^{\prime} e^{-i \beta_{j} z} \\
& \frac{d}{d z} \widehat{b}_{\gamma}(\omega, z)=-\sqrt{\epsilon} \frac{i k^{2}(\omega)}{2} \sum_{l=1}^{N(\omega)} \frac{C_{\gamma l}^{\omega}(z)}{\sqrt{\sqrt{\gamma} \beta_{l}}}\left(\widehat{a}_{l}(\omega, z) e^{i\left(\beta_{l}+\sqrt{\gamma}\right) z}+\widehat{b}_{l}(\omega, z) e^{-i\left(\beta_{l}-\sqrt{\gamma}\right) z}\right) \\
& -\sqrt{\epsilon} \frac{i k^{2}(\omega)}{2} \int_{\xi}^{k^{2}(\omega)} \frac{C_{\gamma \gamma^{\prime}}^{\omega}(z)}{\gamma^{1 / 4}{\gamma^{\prime}}^{1 / 4}}\left(\widehat{a}_{\gamma^{\prime}}(\omega, z) e^{i\left(\sqrt{\gamma^{\prime}}+\sqrt{\gamma}\right) z}+\widehat{b}_{\gamma^{\prime}}(\omega, z) e^{-i\left(\sqrt{\gamma^{\prime}}-\sqrt{\gamma}\right) z}\right) d \gamma^{\prime} \\
& -\sqrt{\epsilon} \frac{i k^{2}(\omega)}{2} \int_{-\infty}^{-\xi} \frac{C_{\gamma \gamma^{\prime}}^{\omega}(z)}{\gamma^{1 / 4}} \widehat{p}_{\gamma^{\prime}}(\omega, z) d \gamma^{\prime} e^{-i \sqrt{\gamma} z} \\
& \frac{d^{2}}{d z^{2}} \widehat{p}_{\gamma}(\omega, z)+\gamma \widehat{p}_{\gamma}(\omega, z)+\sqrt{\epsilon} g_{\gamma}(\omega, z)=0,
\end{aligned}
$$


where

$$
\begin{aligned}
g_{\gamma}(\omega, z)= & k^{2}(\omega) \sum_{l=1}^{N(\omega)} \frac{C_{\gamma l}^{\omega}(z)}{\sqrt{\beta_{l}}}\left(\widehat{a}_{l}(\omega, z) e^{i \beta_{l} z}+\widehat{b}_{l}(\omega, z) e^{-i \beta_{l} z}\right) \\
& +k^{2}(\omega) \int_{\xi}^{k^{2}(\omega)} \frac{C_{\gamma \gamma^{\prime}}^{\omega}(z)}{\gamma^{\prime 1 / 4}}\left(\widehat{a}_{\gamma^{\prime}}(\omega, z) e^{i \sqrt{\gamma^{\prime}} z}+\widehat{b}_{\gamma^{\prime}}(\omega, z) e^{-i \sqrt{\gamma^{\prime}} z}\right) d \gamma^{\prime} \\
& +k^{2}(\omega) \int_{-\infty}^{-\xi} C_{\gamma \gamma^{\prime}}^{\omega}(z) \widehat{p}_{\gamma^{\prime}}(\omega, z) d \gamma^{\prime} .
\end{aligned}
$$

Let us note that in absence of random perturbations, the amplitudes $\widehat{a}(\omega, z)$ and $\widehat{b}(\omega, z)$ are constant.

We assume that a pulse is emitted at the source plane $L_{S}<0$ and propagates toward the randomly perturbed slab $[0, L / \epsilon]$. Using the previous section, the form of this incident field at $z=0$ is given by

$$
\begin{aligned}
\widehat{p}(\omega, x, 0)= & \sum_{j=1}^{N(\omega)} \frac{\widehat{a}_{j, 0}(\omega)}{\sqrt{\beta_{j}(\omega)}} \phi_{j}(\omega, x)+\int_{\xi}^{k^{2}(\omega)} \frac{\widehat{a}_{\gamma, 0}(\omega)}{\gamma^{1 / 4}} \phi_{\gamma}(\omega, x) d \gamma \\
& +\int_{-S}^{-\xi} \frac{\widehat{c}_{\gamma, 0}(\omega)}{|\gamma|^{1 / 4}} \phi_{\gamma}(\omega, x) d \gamma .
\end{aligned}
$$

Consequently, by the continuity of the pressure field across the interfaces $z=0$ and $z=L / \epsilon$, the coupled mode system is complemented with the boundary conditions

$$
\widehat{a}(\omega, 0)=\widehat{a}_{0}(\omega) \quad \text { and } \quad \widehat{b}\left(\omega, \frac{L}{\epsilon}\right)=0
$$

in $\mathcal{H}_{\xi}^{\omega}$. For $j \in\{1, \ldots, N(\omega)\}, \widehat{a}_{j, 0}(\omega)$ represents the initial amplitude of the $j$ th propagating mode, and for $\gamma \in\left(\xi, k^{2}(\omega)\right), \widehat{a}_{\gamma, 0}(\omega)$ represents the initial amplitude of the $\gamma$ th radiating mode at $z=0$. Moreover, for $\gamma \in(-S,-\xi), \widehat{c}_{\gamma, 0}(\omega)$ represents the initial amplitude of the $\gamma$ th evanescent mode at $z=0$. The second condition implies that no wave comes from the right (homogeneous) section of the waveguide.

4.2. Energy flux for the propagating and radiating modes. In this section we study the energy flux for the propagating and radiating modes, and the influence of the evanescent modes on this flux.

We begin this section by recalling the radiation condition for the evanescent modes

$$
\lim _{z \rightarrow+\infty}\left\|\Pi_{\omega}(-\infty,-\xi)(\widehat{p}(\omega, ., z))\right\|_{H}^{2}=0 .
$$

This condition means, in the homogeneous right half-space, that the energy carried by the evanescent modes decay as the propagation distance becomes large. From the radiation condition and (4.8), we obtain, for almost every $\gamma \in(-\infty,-\xi)$,

$$
\begin{aligned}
\widehat{p}_{\gamma}(\omega, z)= & \frac{\sqrt{\epsilon}}{2 \sqrt{|\gamma|}} \int_{0}^{z \wedge L / \epsilon} g_{\gamma}(\omega, u) e^{\sqrt{|\gamma|}(u-z)} d u+\frac{\sqrt{\epsilon}}{2 \sqrt{|\gamma|}} \int_{z \wedge L / \epsilon}^{L / \epsilon} g_{\gamma}(\omega, u) e^{\sqrt{|\gamma|}(z-u)} d u \\
& +\phi_{\gamma}\left(\omega, x_{0}\right) e^{-\sqrt{|\gamma|}\left(z-L_{S}\right)} \mathbf{1}_{(-S,-\xi)}(\gamma)
\end{aligned}
$$


$\forall z \in[0,+\infty)$. According to (3.12), the relation (4.11) can be viewed as a perturbation of the form of the evanescent mode without a random perturbation. Using the same arguments as in [8] and [7, Chapter 20], we obtain, $\forall z \in[0, L / \epsilon]$,

$$
\begin{aligned}
\|\widehat{a}(\omega, z)\|_{\mathcal{H}_{\xi}^{\omega}}^{2}-\|\widehat{b}(\omega, z)\|_{\mathcal{H}_{\xi}^{\omega}}^{2}= & \left\|\widehat{a}_{0}(\omega)\right\|_{\mathcal{H}_{\xi}^{\omega}}^{2}-\left\|\widehat{b}_{0}(\omega)\right\|_{\mathcal{H}_{\xi}^{\omega}}^{2}-\frac{\epsilon}{2} \int_{-\infty}^{-\xi} \frac{G_{\gamma}(\omega, z)}{\sqrt{|\gamma|}} d \gamma \\
& -\sqrt{\epsilon} \int_{-S}^{-\xi} \phi_{\gamma}\left(\omega, x_{0}\right) e^{\sqrt{|\gamma|} L_{S}} \int_{0}^{z} \operatorname{Im}\left(\overline{g_{\gamma}(\omega, u)}\right) e^{-\sqrt{|\gamma|} u} d u d \gamma
\end{aligned}
$$

where

$$
G_{\gamma}(\omega, z)=\int_{0}^{z} \int_{z}^{L / \epsilon} \operatorname{Im}\left(\overline{g_{\gamma}(\omega, u)} g_{\gamma}(\omega, v)\right) e^{\sqrt{|\gamma|}(u-v)} d v d u
$$

The last term on the right side of the previous relation has the factor $\phi_{\gamma}\left(\omega, x_{0}\right) e^{\sqrt{|\gamma|} L_{S}}$ which is the form of the evanescent mode at $z=0$ without a random perturbation. Therefore, if $L_{S}$ is far away from 0 and whatever the source (evanescent modes decay exponentially from $L_{S}$ to 0 ), or if there is no excitation of modes $\gamma \in(-\infty,-\xi)$ by the source (that is when $S=\xi$ ), we can get the conservation of the global energy flux for the propagating and radiating modes:

$$
\|\widehat{a}(\omega, L / \epsilon)\|_{\mathcal{H}_{\xi}^{\omega}}^{2}+\|\widehat{b}(\omega, 0)\|_{\mathcal{H}_{\xi}^{\omega}}^{2}=\left\|\widehat{a}_{0}(\omega)\right\|_{\mathcal{H}_{\xi}^{\omega}}^{2} .
$$

However, from (4.12) and even if there are no evanescent modes in (4.10), the local energy flux is not conserved. The energy related to the evanescent modes is given by the last two terms on the right side in (4.12). The energy carried by the evanescent modes over the section $[0, L / \epsilon]$ is at most of order

$$
\sqrt{\epsilon} \sup _{z \in[0, L]} \sup _{x \in[0, d]}\left|V\left(x, \frac{z}{\epsilon}\right)\right|^{2} \sup _{z \in[0, L / \epsilon]}\|\widehat{a}(\omega, z)\|_{\mathcal{H}_{\xi}^{\omega}}+\|\widehat{b}(\omega, z)\|_{\mathcal{H}_{\xi}^{\omega}}+\|\widehat{p}(\omega, z)\|_{L^{1}(-\infty,-\xi)}
$$

However, it is difficult to get good a priori estimates for

$$
\sup _{z \in[0, L / \epsilon]}\|\widehat{a}(\omega, z)\|_{\mathcal{H}_{\xi}^{\omega}}^{2}+\|\widehat{b}(\omega, z)\|_{\mathcal{H}_{\xi}^{\omega}}^{2}+\|\widehat{p}(\omega, z)\|_{L^{1}(-\infty,-\xi)}^{2} .
$$

For this reason, let us introduce the stopping "time"

$$
L^{\epsilon}=\inf \left(L>0, \quad \sup _{z \in[0, L / \epsilon]}\|\widehat{a}(\omega, z)\|_{\mathcal{H}_{\xi}^{\omega}}^{2}+\|\widehat{b}(\omega, z)\|_{\mathcal{H}_{\xi}^{\omega}}^{2}+\|\widehat{p}(\omega, z)\|_{L^{1}(-\infty,-\xi)}^{2} \geq \frac{1}{\sqrt{\epsilon}}\right) .
$$

The role of this stopping "time" is to limit the size of the random section to ensure that the quantity (4.13) is not too large. Consequently, the energy carried by the evanescent modes over the section $[0, L / \epsilon]$ for $L \leq L^{\epsilon}$, is at most of order $\mathcal{O}\left(\epsilon^{1 / 4} \sup _{z \in[0, L / \epsilon]} \sup _{x \in[0, d]}|V(x, z)|^{2}\right)$, and according to (2.5) the local energy flux for the propagating and the radiating modes is conserved in the asymptotic $\epsilon \rightarrow 0$. More precisely, we can show that $\forall \eta>0$,

$\lim _{\epsilon \rightarrow 0} \mathbb{P}\left(\sup _{z \in[0, L / \epsilon]}\left|\|\widehat{a}(\omega, z)\|_{\mathcal{H}_{\xi}^{\omega}}^{2}-\|\widehat{b}(\omega, z)\|_{\mathcal{H}_{\xi}^{\omega}}^{2}-\left\|\widehat{a}_{0}(\omega)\right\|_{\mathcal{H}_{\xi}^{\omega}}^{2}+\left\|\widehat{b}_{0}(\omega)\right\|_{\mathcal{H}_{\xi}^{\omega}}^{2}\right|>\eta, L \leq L^{\epsilon}\right)=0$.

However, under the forward scattering approximation introduced in Section 4.4, the condition $L \leq L^{\epsilon}$ is readily fulfilled in the limit $\epsilon \rightarrow 0$, that is we have $\lim _{\epsilon \rightarrow 0} \mathbb{P}\left(L^{\epsilon} \leq\right.$ $L)=0$. 
4.3. Influence of the evanescent modes on the propagating and radiating modes. In this section we analyze the influence of the evanescent modes on the coupling mechanism between the propagating and the radiating modes.

First of all, we recall that $\Theta_{\omega} \circ \Pi_{\omega}(-\infty,-\xi)(\widehat{p}(\omega, ., z))$ represents the evanescent part of the pressure field $\widehat{p}(\omega, ., z)$, where $\Theta_{\omega}$ and $\Pi_{\omega}$ are defined in Section 3.1. In this section we consider the Banach space $F=L^{1}(-\infty,-\xi)$ equipped with the norm

$$
\|y\|_{F}=\int_{-\infty}^{-\xi}\left|y_{\gamma}\right| d \gamma
$$

Substituting (4.9) into (4.11), we obtain

$$
\left(I d-\sqrt{\epsilon} \Phi^{\omega}\right)\left(\Theta_{\omega} \circ \Pi_{\omega}(-\infty,-\xi)(\widehat{p}(\omega, . .))\right)=\sqrt{\epsilon} \tilde{p}(\omega, .)+\tilde{p}_{0}(\omega, .) .
$$

This equation holds in the Banach space $\left(\mathcal{C}([0,+\infty), F),\|\cdot\|_{\infty, F}\right)$, where

$$
\|y\|_{\infty, F}=\sup _{z \geq 0}\|y(z)\|_{F}, \quad \forall y \in \mathcal{C}([0,+\infty), F) .
$$

In (4.15), $\Phi^{\omega}$ is a linear bounded operator, from $\left(\mathcal{C}([0,+\infty), F),\|\cdot\|_{\infty, F}\right)$ to itself, defined by

$$
\begin{aligned}
\Phi_{\gamma}^{\omega}(y)(z)= & \frac{k^{2}(\omega)}{2 \sqrt{|\gamma|}} \int_{0}^{z \wedge L / \epsilon} \int_{-\infty}^{-\xi} C_{\gamma \gamma^{\prime}}^{\omega}(u) y_{\gamma^{\prime}}(u) d \gamma^{\prime} e^{\sqrt{|\gamma|}(u-z)} d u \\
& +\frac{k^{2}(\omega)}{2 \sqrt{|\gamma|}} \int_{z \wedge L / \epsilon}^{L / \epsilon} \int_{-\infty}^{-\xi} C_{\gamma \gamma^{\prime}}^{\omega}(u) y_{\gamma^{\prime}}(u) d \gamma^{\prime} e^{\sqrt{|\gamma|}(z-u)} d u
\end{aligned}
$$

$\forall z \in[0,+\infty)$, and, for almost every $\gamma \in(-\infty,-\xi)$,

$$
\begin{aligned}
\tilde{p}_{\gamma}(\omega, z)= & \frac{k^{2}(\omega)}{2 \sqrt{|\gamma|}} \int_{0}^{z \wedge L / \epsilon}\left[\sum_{l=1}^{N(\omega)} \frac{C_{\gamma l}^{\omega}(u)}{\sqrt{\beta_{l}}}\left(\widehat{a}_{l}(\omega, u) e^{i \beta_{l} u}+\widehat{b}_{l}(\omega, u) e^{-i \beta_{l} u}\right)\right. \\
& \left.+\int_{\xi}^{k^{2}(\omega)} \frac{C_{\gamma \gamma^{\prime}}^{\omega}(u)}{\gamma^{\prime / 4}}\left(\widehat{a}_{\gamma^{\prime}}(\omega, u) e^{i \sqrt{\gamma^{\prime}} u}+\widehat{b}_{\gamma^{\prime}}(\omega, u) e^{-i \sqrt{\gamma^{\prime} u}}\right)\right] d \gamma^{\prime} e^{\sqrt{|\gamma|}(u-z)} d u \\
+ & \frac{k^{2}(\omega)}{2 \sqrt{|\gamma|}} \int_{z \wedge L / \epsilon}^{L / \epsilon}\left[\sum_{l=1}^{N(\omega)} \frac{C_{\gamma l}^{\omega}(u)}{\sqrt{\beta_{l}}}\left(\widehat{a}_{l}(\omega, u) e^{i \beta_{l} u}+\widehat{b}_{l}(\omega, u) e^{-i \beta_{l} u}\right)\right. \\
& \left.+\int_{\xi}^{k^{2}(\omega)} \frac{C_{\gamma \gamma^{\prime}}^{\omega}(u)}{\gamma^{\prime 1 / 4}}\left(\widehat{a}_{\gamma^{\prime}}(\omega, u) e^{i \sqrt{\gamma^{\prime}} u}+\widehat{b}_{\gamma^{\prime}}(\omega, u) e^{-i \sqrt{\gamma^{\prime}} u}\right)\right] d \gamma^{\prime} e^{\sqrt{|\gamma|}(z-u)} d u
\end{aligned}
$$

$\forall z \in[0,+\infty)$. Finally, for almost every $\gamma \in(-\infty,-\xi)$ and $\forall z \in[0,+\infty)$,

$$
\tilde{p}_{\gamma, 0}(\omega, z)=\phi_{\gamma}\left(\omega, x_{0}\right) e^{-\sqrt{|\gamma|}\left(z-L_{S}\right)} \mathbf{1}_{(-S,-\xi)}(\gamma) .
$$

We remark that $\Theta_{\omega} \circ \Pi_{\omega}(-\infty,-\xi)(\widehat{p}(\omega, . .).) \in \mathcal{C}([0,+\infty), F)$ by (4.3). Moreover, $\tilde{p}(\omega,.) \in \mathcal{C}([0,+\infty), F)$ since $\int_{-\infty}^{-\xi} \frac{A_{\gamma}(\omega)}{|\gamma|} d \gamma<+\infty$, where $A_{\gamma}(\omega)$ is defined by $(3.9)$ and satisfies (3.10). We can check that the norm of the operator $\Phi^{\omega}$ is bounded by

$$
\left\|\Phi^{\omega}\right\| \leq K(\xi, d) \sup _{z \in[0, L / \epsilon]} \sup _{x \in[0, d]}|V(x, z)| .
$$


Consequently, using (2.5), $\lim _{\epsilon \rightarrow 0} \mathbb{P}\left(I d-\sqrt{\epsilon} \Phi^{\omega}\right.$ is invertible $)=1$. Then, the condition ( $I d-\sqrt{\epsilon} \Phi^{\omega}$ is invertible) is satisfied in the asymptotic $\epsilon \rightarrow 0$. As a result, on the event $\left(I d-\sqrt{\epsilon} \Phi^{\omega}\right.$ is invertible), and using (4.4), (4.5), (4.6), and (4.7), we have

$$
\begin{aligned}
\Theta_{\omega} \circ \Pi_{\omega}(-\infty,-\xi)(\widehat{p}(\omega, . . .))=\sqrt{\epsilon} \tilde{p}_{2}(\omega, .)+\tilde{p}_{0}(\omega, .)+\sqrt{\epsilon} \Phi^{\omega}\left(\tilde{p}_{0}(\omega, .)\right) \\
\quad+\mathcal{O}\left(\epsilon \sup _{z \in[0, L / \epsilon]} \sup _{x \in[0, d]}|V(x, z)|^{2} \sup _{z \in[0, L / \epsilon]}\|\widehat{a}(\omega, z)\|_{\mathcal{H}_{\xi}^{\omega}}+\|\widehat{b}(\omega, z)\|_{\mathcal{H}_{\xi}^{\omega}}+\|\widehat{p}(\omega, z)\|_{F}\right)
\end{aligned}
$$

in $\mathcal{C}([0,+\infty), F)$, where

$$
\begin{aligned}
& \tilde{p}_{\gamma, 2}(\omega, z)=\frac{k^{2}(\omega)}{2 \sqrt{|\gamma|}} \int_{0}^{z \wedge L / \epsilon}\left[\sum_{l=1}^{N(\omega)} \frac{C_{\gamma l}(u)}{\sqrt{\beta_{l}}}\left(\widehat{a}_{l}(\omega, z \wedge L / \epsilon) e^{i \beta_{l} u}+\widehat{b}_{l}(\omega, z \wedge L / \epsilon) e^{-i \beta_{l} u}\right)\right. \\
& \left.+\int_{\xi}^{k^{2}(\omega)} \frac{C_{\gamma \gamma^{\prime}}(u)}{\gamma^{\prime 1 / 4}}\left(\widehat{a}_{\gamma^{\prime}}(\omega, z \wedge L / \epsilon) e^{i \sqrt{\gamma^{\prime} u}}+\widehat{b}_{\gamma^{\prime}}(\omega, z \wedge L / \epsilon) e^{-i \sqrt{\gamma^{\prime}} u}\right)\right] d \gamma^{\prime} e^{\sqrt{|\gamma|}(u-z)} d u \\
& +\frac{k^{2}(\omega)}{2 \sqrt{|\gamma|}} \int_{z \wedge L / \epsilon}^{L / \epsilon}\left[\sum_{l=1}^{N(\omega)} \frac{C_{\gamma l}(u)}{\sqrt{\beta_{l}}}\left(\widehat{a}_{l}(\omega, z \wedge L / \epsilon) e^{i \beta_{l} u}+\widehat{b}_{l}(\omega, z \wedge L / \epsilon) e^{-i \beta_{l} u}\right)\right. \\
& \left.+\int_{\xi}^{k^{2}(\omega)} \frac{C_{\gamma \gamma^{\prime}}(u)}{\gamma^{\prime 1 / 4}}\left(\widehat{a}_{\gamma^{\prime}}(\omega, z \wedge L / \epsilon) e^{i \sqrt{\gamma^{\prime}} u}+\widehat{b}_{\gamma^{\prime}}(\omega, z \wedge L / \epsilon) e^{-i \sqrt{\gamma^{\prime}} u}\right)\right] d \gamma^{\prime} e^{\sqrt{|\gamma|}(z-u)} d u
\end{aligned}
$$

$\forall z \in[0,+\infty)$. Consequently, we can rewrite (4.4), (4.5), (4.6), and (4.7) in a closed form in $\mathcal{H}_{\xi}^{\omega} \times \mathcal{H}_{\xi}^{\omega} . \forall z \in[0, L / \epsilon]$, we obtain

$$
\begin{aligned}
& \frac{d}{d z} \widehat{a}(\omega, z)=\sqrt{\epsilon} \mathbf{H}^{a a}(\omega, z)(\widehat{a}(\omega, z))+\sqrt{\epsilon} \mathbf{H}^{a b}(\omega, z)(\widehat{b}(\omega, z))+\sqrt{\epsilon} \mathbf{R}^{a, L_{S}}(\omega, z) \\
& \left.\quad+\epsilon \mathbf{G}^{a a}(\omega, z)(\widehat{a}(\omega, z))+\epsilon \mathbf{G}^{a b}(\omega, z) \widehat{b}(\omega, z)\right)+\epsilon \tilde{\mathbf{R}}^{a, L_{S}}(\omega, z) \\
& \quad+\mathcal{O}\left(\epsilon^{3 / 2} \sup _{z \in[0, L / \epsilon]} \sup _{x \in[0, d]}|V(x, z)|^{2} \sup _{z \in[0, L / \epsilon]}\|\widehat{a}(\omega, z)\|_{\mathcal{H}_{\xi}^{\omega}}+\|\widehat{b}(\omega, z)\|_{\mathcal{H}_{\xi}^{\omega}}+\|\widehat{p}(\omega, z)\|_{F}\right), \\
& \left.\frac{d}{d z} \widehat{b}(\omega, z)=\sqrt{\epsilon} \mathbf{H}^{b a}(\omega, z)(\widehat{a}(\omega, z))+\sqrt{\epsilon} \mathbf{H}^{b b}(\omega, z) \widehat{b}(\omega, z)\right)+\sqrt{\epsilon} \mathbf{R}^{b, L_{S}}(\omega, z) \\
& \left.\quad+\epsilon \mathbf{G}^{b a}(\omega, z)(\widehat{a}(\omega, z))+\epsilon \mathbf{G}^{b b}(\omega, z) \widehat{b}(\omega, z)\right)+\epsilon \tilde{\mathbf{R}}^{b, L_{S}}(\omega, z) \\
& \quad+\mathcal{O}\left(\epsilon^{3 / 2} \sup _{z \in[0, L / \epsilon]} \sup _{x \in[0, d]}|V(x, z)|^{2} \sup _{z \in[0, L / \epsilon]}\|\widehat{a}(\omega, z)\|_{\mathcal{H}_{\xi}^{\omega}}+\|\widehat{b}(\omega, z)\|_{\mathcal{H}_{\xi}^{\omega}}+\|\widehat{p}(\omega, z)\|_{F}\right) .
\end{aligned}
$$

Let us recall that these equations hold on the event $\left(I d-\sqrt{\epsilon} \Phi^{\omega}\right.$ is invertible) with $\lim _{\epsilon \rightarrow 0} \mathbb{P}\left(I d-\sqrt{\epsilon} \Phi^{\omega}\right.$ is invertible $)=1$. In these equations, $\mathbf{H}^{a a}(\omega, z), \mathbf{H}^{a b}(\omega, z)$, $\mathbf{H}^{b a}(\omega, z), \mathbf{H}^{b b}(\omega, z), \mathbf{G}^{a a}(\omega, z), \mathbf{G}^{a b}(\omega, z), \mathbf{G}^{b a}(\omega, z)$ and $\mathbf{G}^{b b}(\omega, z)$ are operators from $\mathcal{H}_{\xi}^{\omega}$ to itself defined by:

$$
\begin{aligned}
\mathbf{H}_{j}^{a a}(\omega, z)(y)=\overline{\mathbf{H}_{j}^{b b}(\omega, z)}(y)= & \frac{i k^{2}(\omega)}{2}\left[\sum_{l=1}^{N(\omega)} \frac{C_{j l}^{\omega}(z)}{\sqrt{\beta_{j}(\omega) \beta_{l}(\omega)}} y_{l} e^{i\left(\beta_{l}(\omega)-\beta_{j}(\omega)\right) z}\right. \\
& \left.+\int_{\xi}^{k^{2}(\omega)} \frac{C_{j \gamma^{\prime}}^{\omega}(z)}{\sqrt{\beta_{j}(\omega) \sqrt{\gamma^{\prime}}}} y_{\gamma^{\prime}} e^{i\left(\sqrt{\gamma^{\prime}}-\beta_{j}(\omega)\right) z} d \gamma^{\prime}\right]
\end{aligned}
$$




$$
\begin{aligned}
& \mathbf{H}_{\gamma}^{a a}(\omega, z)(y)=\overline{\mathbf{H}_{\gamma}^{b b}(\omega, z)}(y)=\frac{i k^{2}(\omega)}{2}\left[\sum_{l=1}^{N(\omega)} \frac{C_{\gamma l}^{\omega}(z)}{\sqrt{\sqrt{\gamma} \beta_{l}(\omega)}} y_{l} e^{i\left(\beta_{l}(\omega)-\sqrt{\gamma}\right) z}\right. \\
& \left.+\int_{\xi}^{k^{2}(\omega)} \frac{C_{\gamma \gamma^{\prime}}^{\omega}(z)}{\gamma^{1 / 4} \gamma^{\prime 1 / 4}} y_{\gamma^{\prime}} e^{i\left(\sqrt{\gamma^{\prime}}-\sqrt{\gamma}\right) z} d \gamma^{\prime}\right] \\
& \begin{aligned}
\mathbf{H}_{j}^{a b}(\omega, z)(y)=\overline{\mathbf{H}_{j}^{b a}(\omega, z)}(y)= & \frac{i k^{2}(\omega)}{2}\left[\sum_{l=1}^{N(\omega)} \frac{C_{j l}^{\omega}(z)}{\sqrt{\beta_{j}(\omega) \beta_{l}(\omega)}} y_{l} e^{-i\left(\beta_{l}(\omega)+\beta_{j}(\omega)\right) z}\right. \\
& \left.+\int_{\xi}^{k^{2}(\omega)} \frac{C_{j \gamma^{\prime}}^{\omega}(z)}{\sqrt{\beta_{j}(\omega) \sqrt{\gamma^{\prime}}}} y_{\gamma^{\prime}} e^{-i\left(\sqrt{\gamma^{\prime}}+\beta_{j}(\omega)\right) z} d \gamma^{\prime}\right]
\end{aligned} \\
& \mathbf{H}_{\gamma}^{a b}(\omega, z)(y)=\overline{\mathbf{H}_{\gamma}^{b a}(\omega, z)}(y)=\frac{i k^{2}(\omega)}{2}\left[\sum_{l=1}^{N(\omega)} \frac{C_{\gamma l}^{\omega}(z)}{\sqrt{\sqrt{\gamma} \beta_{l}(\omega)}} y_{l} e^{-i\left(\beta_{l}(\omega)+\sqrt{\gamma}\right) z}\right. \\
& \left.+\int_{\xi}^{k^{2}(\omega)} \frac{C_{\gamma \gamma^{\prime}}(z)}{\gamma^{1 / 4} \gamma^{\prime / 4}} y_{\gamma^{\prime}} e^{-i\left(\sqrt{\gamma^{\prime}}+\sqrt{\gamma}\right) z} d \gamma^{\prime}\right]
\end{aligned}
$$

$$
\begin{aligned}
& \mathbf{G}_{j}^{a a}(\omega, z)(y)=\overline{\mathbf{G}_{j}^{b b}(\omega, z)}(y) \\
& =\frac{i k^{4}(\omega)}{4}\left[\sum _ { l = 1 } ^ { N ( \omega ) } \int _ { - \infty } ^ { - \xi } \left[\int_{0}^{z} \frac{C_{j \gamma^{\prime}}^{\omega}(z) C_{\gamma^{\prime} l}^{\omega}(u)}{\sqrt{\beta_{j}(\omega)\left|\gamma^{\prime}\right| \beta_{l}(\omega)}} e^{i \beta_{l}(\omega) u-\sqrt{\left|\gamma^{\prime}\right|}(z-u)} d u\right.\right. \\
& \left.\left.+\int_{z}^{L / \epsilon} \frac{C_{j \gamma^{\prime}}^{\omega}(z) C_{\gamma^{\prime} l}^{\omega}(u)}{\sqrt{\beta_{j}(\omega)\left|\gamma^{\prime}\right| \beta_{l}(\omega)}} e^{i \beta_{l}(\omega) u-\sqrt{\left|\gamma^{\prime}\right|}(u-z)} d u\right] d \gamma^{\prime} e^{-i \beta_{j}(\omega) z} y_{l}\right] \\
& +\frac{i k^{4}(\omega)}{4}\left[\int _ { \xi } ^ { k ^ { 2 } ( \omega ) } \int _ { - \infty } ^ { - \xi } \left[\int_{0}^{z} \frac{C_{j \gamma^{\prime}}^{\omega}(z) C_{\gamma^{\prime} \gamma^{\prime \prime}}^{\omega}(u)}{\sqrt{\beta_{j}(\omega)\left|\gamma^{\prime}\right| \sqrt{\gamma^{\prime \prime}}}} e^{i \sqrt{\gamma^{\prime \prime}} u-\sqrt{\left|\gamma^{\prime}\right|}(z-u)} d u\right.\right. \\
& \left.\left.+\int_{z}^{L / \epsilon} \frac{C_{j \gamma^{\prime}}^{\omega}(z) C_{\gamma^{\prime} \gamma^{\prime \prime}}^{\omega}(u)}{\sqrt{\beta_{j}(\omega)\left|\gamma^{\prime}\right| \sqrt{\gamma^{\prime \prime}}}} e^{i \sqrt{\gamma^{\prime \prime}} u-\sqrt{\left|\gamma^{\prime}\right|}(u-z)} d u\right] d \gamma^{\prime} e^{-i \beta_{j}(\omega) z} y_{\gamma^{\prime \prime}} d \gamma^{\prime \prime}\right] \\
& \mathbf{G}_{\gamma}^{a a}(\omega, z)(y)=\overline{\mathbf{G}_{\gamma}^{b b}(\omega, z)}(y) \\
& =\frac{i k^{4}(\omega)}{4}\left[\sum _ { l = 1 } ^ { N ( \omega ) } \int _ { - \infty } ^ { - \xi } \left[\int_{0}^{z} \frac{C_{\gamma \gamma^{\prime}}^{\omega}(z) C_{\gamma^{\prime} l}^{\omega}(u)}{\sqrt{\sqrt{\gamma}\left|\gamma^{\prime}\right| \beta_{l}(\omega)}} e^{i \beta_{l}(\omega) u-\sqrt{\left|\gamma^{\prime}\right|}(z-u)} d u\right.\right. \\
& \left.\left.+\int_{z}^{L / \epsilon} \frac{C_{\gamma \gamma^{\prime}}^{\omega}(z) C_{\gamma^{\prime} l}^{\omega}(u)}{\sqrt{\sqrt{\gamma}\left|\gamma^{\prime}\right| \beta_{l}(\omega)}} e^{i \beta_{l}(\omega) u-\sqrt{\left|\gamma^{\prime}\right|}(u-z)} d u\right] d \gamma^{\prime} e^{-i \sqrt{\gamma} z} y_{l}\right] \\
& +\frac{i k^{4}(\omega)}{4}\left[\int _ { \xi } ^ { k ^ { 2 } ( \omega ) } \int _ { - \infty } ^ { - \xi } \left[\int_{0}^{z} \frac{C_{\gamma \gamma^{\prime}}^{\omega}(z) C_{\gamma^{\prime} \gamma^{\prime \prime}}^{\omega}(u)}{\sqrt{\sqrt{\gamma}\left|\gamma^{\prime}\right| \sqrt{\gamma^{\prime \prime}}}} e^{i \sqrt{\gamma^{\prime \prime}} u-\sqrt{\left|\gamma^{\prime}\right|}(z-u)} d u\right.\right. \\
& \left.\left.+\int_{z}^{L / \epsilon} \frac{C_{\gamma \gamma^{\prime}}^{\omega}(z) C_{\gamma^{\prime} \gamma^{\prime \prime}}^{\omega}(u)}{\sqrt{\sqrt{\gamma}\left|\gamma^{\prime}\right| \sqrt{\gamma^{\prime \prime}}}} e^{i \sqrt{\gamma^{\prime \prime}} u-\sqrt{\left|\gamma^{\prime}\right|}(u-z)} d u\right] d \gamma^{\prime} e^{-i \sqrt{\gamma} z} y_{\gamma^{\prime \prime}} d \gamma^{\prime \prime}\right]
\end{aligned}
$$




$$
\begin{aligned}
& \mathbf{G}_{j}^{a b}(\omega, z)(y)=\overline{\mathbf{G}_{j}^{b a}(\omega, z)}(y) \\
& =\frac{i k^{4}(\omega)}{4}\left[\sum _ { l = 1 } ^ { N ( \omega ) } \int _ { - \infty } ^ { - \xi } \left[\int_{0}^{z} \frac{C_{j \gamma^{\prime}}^{\omega}(z) C_{\gamma^{\prime} l}^{\omega}(u)}{\sqrt{\beta_{j}(\omega)\left|\gamma^{\prime}\right| \beta_{l}(\omega)}} e^{-i \beta_{l}(\omega) u-\sqrt{\left|\gamma^{\prime}\right|}(z-u)} d u\right.\right. \\
& \left.\left.+\int_{z}^{L / \epsilon} \frac{C_{j \gamma^{\prime}}^{\omega}(z) C_{\gamma^{\prime} l}^{\omega}(u)}{\sqrt{\beta_{j}(\omega)\left|\gamma^{\prime}\right| \beta_{l}(\omega)}} e^{-i \beta_{l}(\omega) u-\sqrt{\left|\gamma^{\prime}\right|}(u-z)} d u\right] d \gamma^{\prime} e^{-i \beta_{j}(\omega) z} y_{l}\right] \\
& +\frac{i k^{4}(\omega)}{4}\left[\int _ { \xi } ^ { k ^ { 2 } ( \omega ) } \int _ { - \infty } ^ { - \xi } \left[\int_{0}^{z} \frac{C_{j \gamma^{\prime}}^{\omega}(z) C_{\gamma^{\prime} \gamma^{\prime \prime}}^{\omega}(u)}{\sqrt{\beta_{j}(\omega)\left|\gamma^{\prime}\right| \sqrt{\gamma^{\prime \prime}}}} e^{-i \sqrt{\gamma^{\prime \prime}} u-\sqrt{\left|\gamma^{\prime}\right|}(z-u)} d u\right.\right. \\
& \left.\left.+\int_{z}^{L / \epsilon} \frac{C_{j \gamma^{\prime}}^{\omega}(z) C_{\gamma^{\prime} \gamma^{\prime \prime}}^{\omega}(u)}{\sqrt{\beta_{j}(\omega)\left|\gamma^{\prime}\right| \sqrt{\gamma^{\prime \prime}}}} e^{-i \sqrt{\gamma^{\prime \prime}} u-\sqrt{\left|\gamma^{\prime}\right|}(u-z)} d u\right] d \gamma^{\prime} e^{-i \beta_{j}(\omega) z} y_{\gamma^{\prime \prime}} d \gamma^{\prime \prime}\right], \\
& \mathbf{G}_{\gamma}^{a b}(\omega, z)(y)=\overline{\mathbf{G}_{\gamma}^{b a}(\omega, z)}(y) \\
& =\frac{i k^{4}(\omega)}{4}\left[\sum _ { l = 1 } ^ { N ( \omega ) } \int _ { - \infty } ^ { - \xi } \left[\int_{0}^{z} \frac{C_{\gamma \gamma^{\prime}}^{\omega}(z) C_{\gamma^{\prime} l}^{\omega}(u)}{\sqrt{\sqrt{\gamma}\left|\gamma^{\prime}\right| \beta_{l}(\omega)}} e^{-i \beta_{l}(\omega) u-\sqrt{\left|\gamma^{\prime}\right|}(z-u)} d u\right.\right. \\
& \left.\left.+\int_{z}^{L / \epsilon} \frac{C_{\gamma \gamma^{\prime}}^{\omega}(z) C_{\gamma^{\prime} l}^{\omega}(u)}{\sqrt{\sqrt{\gamma}\left|\gamma^{\prime}\right| \beta_{l}(\omega)}} e^{-i \beta_{l}(\omega) u-\sqrt{\left|\gamma^{\prime}\right|}(u-z)} d u\right] d \gamma^{\prime} e^{-i \sqrt{\gamma} z} y_{l}\right] \\
& +\frac{i k^{4}(\omega)}{4}\left[\int _ { \xi } ^ { k ^ { 2 } ( \omega ) } \int _ { - \infty } ^ { - \xi } \left[\int_{0}^{z} \frac{C_{\gamma \gamma^{\prime}}^{\omega}(z) C_{\gamma^{\prime} \gamma^{\prime \prime}}^{\omega}(u)}{\sqrt{\sqrt{\gamma}\left|\gamma^{\prime}\right| \sqrt{\gamma^{\prime \prime}}}} e^{-i \sqrt{\gamma^{\prime \prime}} u-\sqrt{\left|\gamma^{\prime}\right|}(z-u)} d u\right.\right. \\
& \left.\left.+\int_{z}^{L / \epsilon} \frac{C_{\gamma \gamma^{\prime}}^{\omega}(z) C_{\gamma^{\prime} \gamma^{\prime \prime}}^{\omega}(u)}{\sqrt{\sqrt{\gamma}\left|\gamma^{\prime}\right| \sqrt{\gamma^{\prime \prime}}}} e^{-i \sqrt{\gamma^{\prime \prime}} u-\sqrt{\left|\gamma^{\prime}\right|}(u-z)} d u\right] d \gamma^{\prime} e^{-i \sqrt{\gamma} z} y_{\gamma^{\prime \prime}} d \gamma^{\prime \prime}\right] .
\end{aligned}
$$

The operators $\mathbf{H}^{a a}(\omega, z)$ and $\mathbf{H}^{a b}(\omega, z)$ represent the coupling between the propagating and the radiating modes with themselves, while the operators $\mathbf{G}^{a a}(\omega, z)$ and $\mathbf{G}^{a b}(\omega, z)$ represent the coupling between the evanescent modes with the propagating and the radiating modes. Moreover, $\mathbf{R}^{a, L_{S}}(\omega, z), \tilde{\mathbf{R}}^{a, L_{S}}(\omega, z), \mathbf{R}^{b, L_{S}}(\omega, z)$, and $\tilde{\mathbf{R}}^{b, L_{S}}(\omega, z)$ represent the influence of the evanescent modes produced by the source term on the propagating and the radiating modes. These terms are defined by

$$
\begin{aligned}
& \mathbf{R}_{j}^{a, L_{S}}(\omega, z)=\overline{\mathbf{R}_{j}^{b, L_{S}}(\omega, z)}=\frac{i k^{2}(\omega)}{2} \int_{-S}^{-\xi} \frac{C_{j \gamma^{\prime}}^{\omega}(z)}{\sqrt{\beta_{j}(\omega)}} \phi_{\gamma^{\prime}}\left(\omega, x_{0}\right) e^{-\sqrt{\left|\gamma^{\prime}\right|}\left(z-L_{S}\right)} d \gamma^{\prime} e^{-i \beta_{j}(\omega) z},(4.24) \\
& \mathbf{R}_{\gamma}^{a, L_{S}}(\omega, z)=\overline{\mathbf{R}_{\gamma}^{b, L_{S}}(\omega, z)}=\frac{i k^{2}(\omega)}{2} \int_{-S}^{-\xi} \frac{C_{\gamma \gamma^{\prime}}^{\omega}(z)}{|\gamma|^{\mid / 4}} \phi_{\gamma^{\prime}}\left(\omega, x_{0}\right) e^{-\sqrt{\left|\gamma^{\prime}\right|\left(z-L_{S}\right)}} d \gamma^{\prime} e^{-i \sqrt{\gamma} z},(4.25) \\
& =\frac{i k^{4}(\omega)}{4} \int_{-\infty}^{-\xi} \int_{-S}^{-\xi}\left[\int_{0}^{z, L_{S}} \frac{C_{j \gamma^{\prime}}^{\omega}(z) C_{\gamma^{\prime} \gamma^{\prime \prime}}^{\omega}(u)}{\sqrt{\beta_{j}(\omega)\left|\gamma^{\prime}\right|}} \phi_{\gamma^{\prime \prime}}\left(\omega, x_{0}\right) e^{-\sqrt{\left|\gamma^{\prime \prime}\right|}\left(u-L_{S}\right)} e^{-\sqrt{\left|\gamma^{\prime}\right|}(z-u)} d u\right. \\
& \left.+\int_{z}^{L / \epsilon} \frac{C_{j \gamma^{\prime}}^{\omega}(z) C_{\gamma^{\prime} \gamma^{\prime \prime}}^{\omega}(u)}{\sqrt{\beta_{j}(\omega)\left|\gamma^{\prime}\right|}} \phi_{\gamma^{\prime \prime}}\left(\omega, x_{0}\right) e^{-\sqrt{\left|\gamma^{\prime \prime}\right|}\left(u-L_{S}\right)} e^{-\sqrt{\left|\gamma^{\prime}\right|}(u-z)} d u\right] d \gamma^{\prime \prime} d \gamma^{\prime} e^{-i \beta_{j}(\omega) z},
\end{aligned}
$$




$$
\begin{aligned}
& \tilde{\mathbf{R}}_{\gamma}^{a, L_{S}}(\omega, z)=\overline{\tilde{\mathbf{R}}_{\gamma}^{b, L_{S}}(\omega, z)} \\
= & \frac{i k^{4}(\omega)}{4} \int_{-\infty}^{-\xi} \int_{-S}^{-\xi}\left[\int_{0}^{z} \frac{C_{\gamma \gamma^{\prime}}^{\omega}(z) C_{\gamma^{\prime} \gamma^{\prime \prime}}^{\omega}(u)}{\sqrt{\sqrt{\gamma}\left|\gamma^{\prime}\right|}} \phi_{\gamma^{\prime \prime}}\left(\omega, x_{0}\right) e^{-\sqrt{\left|\gamma^{\prime \prime}\right|}\left(u-L_{S}\right)} e^{-\sqrt{\left|\gamma^{\prime}\right|}(z-u)} d u\right. \\
& \left.+\int_{z}^{L / \epsilon} \frac{C_{\gamma \gamma^{\prime}}^{\omega}(z) C_{\gamma^{\prime} \gamma^{\prime \prime}}^{\omega}(u)}{\sqrt{\sqrt{\gamma}\left|\gamma^{\prime}\right|}} \phi_{\gamma^{\prime \prime}}\left(\omega, x_{0}\right) e^{-\sqrt{\left|\gamma^{\prime \prime}\right|}\left(u-L_{S}\right)} e^{-\sqrt{\left|\gamma^{\prime}\right|}(u-z)} d u\right] d \gamma^{\prime \prime} d \gamma^{\prime} e^{-i \sqrt{\gamma} z}
\end{aligned}
$$

4.4. Forward scattering approximation. In this section we introduce the forward scattering approximation, which is widely used in the literature. In this approximation the coupling between forward- and backward-propagating modes is assumed to be negligible compared to the coupling between the forward-propagating modes. We refer to $[9,11]$ for justifications on the validity of this approximation.

The justification of this approximation is as follows: the coupling between a rightgoing propagating mode and a left-going propagating mode involves a coefficient of the form

$$
\int_{0}^{+\infty} \mathbb{E}\left[C_{j l}^{\omega}(0) C_{j l}^{\omega}(z)\right] \cos \left(\left(\beta_{l}(\omega)+\beta_{j}(\omega)\right) z\right) d z,
$$

and the coupling between two right-going propagating modes or two left-going propagating modes involves a coefficient of the form

$$
\int_{0}^{+\infty} \mathbb{E}\left[C_{j l}^{\omega}(0) C_{j l}^{\omega}(z)\right] \cos \left(\left(\beta_{l}(\omega)-\beta_{j}(\omega)\right) z\right) d z,
$$

$\forall(j, l) \in\{1, \ldots, N(\omega)\}^{2}$. Therefore, if we assume that

$$
\int_{0}^{+\infty} \mathbb{E}\left[C_{j l}^{\omega}(0) C_{j l}^{\omega}(z)\right] \cos \left(\left(\beta_{l}(\omega)+\beta_{j}(\omega)\right) z\right) d z=0, \quad \forall(j, l) \in\{1, \ldots, N(\omega)\}^{2},
$$

then there is no coupling between right-going and left-going propagating modes, which justifies the forward scattering approximation, but there is still coupling between right-going propagating modes which will be described in Section 5 .

In our context the operator $R(\omega)$, introduced in Section 3.1, has a continuous spectrum and it becomes technically complex to apply a limit theorem for the rescaled process $(\widehat{a}(\omega, z / \epsilon), \widehat{b}(\omega, z / \epsilon))$. The reason is the following: this process is not bounded and the stopping times which are the first exit times of closed balls are not lower semicontinuous for the topology of $\mathcal{C}\left([0, L], \mathcal{H}_{\xi, w}^{\omega}\right)$, where $\mathcal{H}_{\xi, w}^{\omega}$ stands for $\mathcal{H}_{\xi}^{\omega}$ equipped with the weak topology. In our context the continuous part $\left(\xi, k^{2}(\omega)\right)$ of the spectrum imposes us to use the norm $\|\cdot\|_{\mathcal{H}_{\xi}^{\omega}}$ to control some quantities. Moreover, according to Theorem 5.4, in which the energy of the limit process is not conserved, it seems not possible to show a limit theorem on $\mathcal{C}\left([0, L],\left(\mathcal{H}_{\xi}^{\omega},\|\cdot\|_{\mathcal{H}_{\xi}}\right)\right)$ in view of (4.14). In [7] and [9] there is a finite number of propagating modes, so that the weak topology and the strong topology are the same. In [11] the number of propagating modes increases as $\epsilon$ goes to 0 . However, in this last case, the problem can be corrected by considering the first exit times of a closed ball related to the weak topology and by considering the process in an appropriate finite-dimensional dual space.

In our context if we forget these technical problems, according to $[7,9]$ the forward scattering approximation should be valid in the asymptotic $\epsilon \rightarrow 0$ under the assumption that the power spectral density of the process $V$, i.e. the Fourier transform of its 
$z$-autocorrelation function, possesses a cut-off wavenumber. In other words, we can consider the case where

$$
\int_{0}^{+\infty} \mathbb{E}\left[C_{j l}^{\omega}(0) C_{j l}^{\omega}(z)\right] \cos \left(\left(\beta_{l}(\omega)+\beta_{j}(\omega)\right) z\right) d z=0, \quad \forall(j, l) \in\{1, \ldots, N(\omega)\}^{2} .
$$

Let us remark that the continuous part $\left(0, k^{2}(\omega)\right)$ of the spectrum, which corresponds to the radiating modes, does not play any role in the previous assumption. The reason is that the radiating part of the process plays no role in the coupling mechanism as we can see in Theorems 5.4 and 5.5 below and therefore remains constant.

Finally, we shall therefore consider the so-called forward equation, that is we consider the simplified equation on $[0, L / \epsilon]$,

$$
\begin{aligned}
& \frac{d}{d z} \widehat{a}(\omega, z)=\sqrt{\epsilon} \mathbf{H}^{a a}(\omega, z)(\widehat{a}(\omega, z))+\sqrt{\epsilon} \mathbf{R}^{a, L_{S}}(\omega, z) \\
& +\epsilon \mathbf{G}^{a a}(\omega, z)(\widehat{a}(\omega, z))+\epsilon \tilde{\mathbf{R}}^{a, L_{S}}(\omega, z) \\
& +\mathcal{O}\left(\epsilon^{3 / 2} \sup _{z \in[0, L / \epsilon]} \sup _{x \in[0, d]}|V(x, z)|^{2} \sup _{z \in[0, L / \epsilon]}\|\widehat{a}(\omega, z)\|_{\mathcal{H}_{\xi}^{\omega}}+\|\widehat{p}(\omega, z)\|_{F}\right)
\end{aligned}
$$

in $\mathcal{H}_{\xi}^{\omega}$.

\section{Coupled mode processes}

In this section, we study the asymptotic behavior, first as $\epsilon \rightarrow 0$, and second as $\xi \rightarrow 0$, of the statistical properties of the coupling mechanism in terms of a diffusion process.

Let us define the rescaled process

$$
\widehat{a}^{\epsilon}(\omega, z)=\widehat{a}\left(\omega, \frac{z}{\epsilon}\right), \quad \forall z \in[0, L] .
$$

This scaling corresponds to the size of the random section $[0, L / \epsilon]$. This process satisfies the rescaled coupled mode equations on $[0, L]$ :

$$
\begin{aligned}
\frac{d}{d z} \widehat{a}^{\epsilon}(\omega, z)= & \frac{1}{\sqrt{\epsilon}} \mathbf{H}^{a a}\left(\omega, \frac{z}{\epsilon}\right)\left(\widehat{a}^{\epsilon}(\omega, z)\right)+\frac{1}{\sqrt{\epsilon}} \mathbf{R}^{a, L_{S}}\left(\omega, \frac{z}{\epsilon}\right) \\
& +\mathbf{G}^{a a}\left(\omega, \frac{z}{\epsilon}\right)\left(\widehat{a}^{\epsilon}(\omega, z)\right)+\tilde{\mathbf{R}}^{a, L_{S}}\left(\omega, \frac{z}{\epsilon}\right) \\
& +\mathcal{O}\left(\sqrt{\epsilon} \sup _{z \in[0, L / \epsilon]} \sup _{x \in[0, d]}|V(x, z)|^{2} \sup _{z \in[0, L]}\left\|\widehat{a}^{\epsilon}(\omega, z)\right\|_{\mathcal{H}_{\xi}^{\omega}}+\|\widehat{p}(\omega, z / \epsilon)\|_{F}\right)
\end{aligned}
$$

in $\mathcal{H}_{\xi}^{\omega}$, with the initial condition $\widehat{a}^{\epsilon}(\omega, 0)=\widehat{a}_{0}(\omega)$. Using Gronwall's inequality, we have the following result [12].

Proposition 5.1. $\forall L>0$,

$$
\lim _{\epsilon \rightarrow 0} \mathbb{P}\left(L^{\epsilon} \leq L\right)=0
$$

where

$$
L^{\epsilon}=\inf \left(L>0, \quad \sup _{z \in[0, L / \epsilon]}\|\widehat{a}(\omega, z)\|_{\mathcal{H} \omega}^{2}+\|\widehat{p}(\omega, z)\|_{F}^{2} \geq \frac{1}{\sqrt{\epsilon}}\right) .
$$

According to Section 4.2, we have, $\forall \eta>0$,

$$
\lim _{\epsilon \rightarrow 0} \mathbb{P}\left(\sup _{z \in[0, L]}\left|\left\|\widehat{a}^{\epsilon}(\omega, z)\right\|_{\mathcal{H}_{\xi}^{\omega}}^{2}-\left\|\widehat{a}_{0}(\omega)\right\|_{\mathcal{H}_{\xi}^{\omega}}^{2}\right|>\eta\right)=0,
$$


that is, the local energy flux for the propagating and the radiating modes is conserved in the asymptotic $\epsilon \rightarrow 0$. As a result, the amplitude $\widehat{a}^{\epsilon}(\omega, z)$ is asymptotically uniformly bounded in the limit $\epsilon \rightarrow 0$ on $[0, L]$.

Let us introduce $\widehat{a}_{1}^{\epsilon}(\omega,$.$) - the unique solution of the differential equation on$ $[0, L]$.

$$
\frac{d}{d z} \widehat{a}_{1}^{\epsilon}(\omega, z)=\frac{1}{\sqrt{\epsilon}} \mathbf{H}^{a a}\left(\omega, \frac{z}{\epsilon}\right)\left(\widehat{a}_{1}^{\epsilon}(\omega, z)\right)+\mathbf{G}^{a a}\left(\omega, \frac{z}{\epsilon}\right)\left(\widehat{a}_{1}^{\epsilon}(\omega, z)\right)
$$

in $\mathcal{H}_{\xi}^{\omega}$, with initial condition $\widehat{a}_{1}^{\epsilon}(\omega, 0)=\widehat{a}_{0}(\omega)$. Using Proposition 5.1, the relation between the solution of the full system (5.1) and the one of the simplified system (5.2) is given by the following proposition [12].

Proposition 5.2.

$$
\forall \eta>0 \text { and } \forall \mu>0, \quad \lim _{\epsilon \rightarrow 0} \mathbb{P}\left(\sup _{z \in[\mu, L]}\left\|\widehat{a}^{\epsilon}(\omega, z)-\widehat{a}_{1}^{\epsilon}(\omega, z)\right\|_{\mathcal{H}_{\xi}^{\omega}}>\eta\right)=0 .
$$

Proposition 5.2 means that the information about the evanescent part of the source profile is lost in the asymptotic $\epsilon \rightarrow 0$. In fact, the coupling mechanism described by the system (5.1) implies that the information about the evanescent part of the source profile is transmitted to the propagating modes through the coefficients $\mathbf{R}^{a, L_{S}}(\omega, z)$ and $\tilde{\mathbf{R}}^{a, L_{S}}(\omega, z)$ defined by (4.24), (4.25), (4.26), and (4.27). In these expressions we have the term $\phi_{\gamma^{\prime}}(\omega, x) e^{-\sqrt{\left|\gamma^{\prime}\right|}\left(z-L_{S}\right)}$, which comes from the right-hand side of (4.11) and which is the form of evanescent modes without a random perturbation. This term is responsible for the loss of information about the evanescent part of the source profile because of its exponentially decreasing behavior.

Finally, we introduce the transfer operator $\mathbf{T}^{\xi, \epsilon}(\omega, z)$ from $\mathcal{H}_{\xi}^{\omega}$ to itself, which is the unique operator solution of the differential equation

$$
\frac{d}{d z} \mathbf{T}^{\xi, \epsilon}(\omega, z)=\frac{1}{\sqrt{\epsilon}} \mathbf{H}^{a a}\left(\omega, \frac{z}{\epsilon}\right) \mathbf{T}^{\xi, \epsilon}(\omega, z)+\mathbf{G}^{a a}\left(\omega, \frac{z}{\epsilon}\right) \mathbf{T}^{\xi, \epsilon}(\omega, z)
$$

with $\mathbf{T}^{\xi, \epsilon}(\omega, 0)=I d$. Then,

$$
\forall z \in[0, L], \quad \widehat{a}_{1}(\omega, z)=\mathbf{T}^{\xi, \epsilon}(\omega, z)\left(\widehat{a}_{0}(\omega)\right),
$$

and we get the following result.

Proposition 5.3.

$$
\forall \eta>0 \quad \text { and } \quad \forall \mu>0, \quad \lim _{\epsilon \rightarrow 0} \mathbb{P}\left(\sup _{z \in[\mu, L]}\left\|\widehat{a}^{\epsilon}(\omega, z)-\mathbf{T}^{\xi, \epsilon}(\omega, z)\left(\widehat{a}_{0}(\omega)\right)\right\|_{\mathcal{H}_{\xi}^{\omega}}^{2}>\eta\right)=0 .
$$

5.1. Limit theorem. This section presents the basic theoretical results of this paper. In [9] and [15], the authors used the limit theorem stated in [24] since the number of propagating modes was fixed. However, in our configuration, in addition to the $N(\omega)$-discrete propagating modes the wave field consists of a continuum of radiating modes. The two following results are based on a diffusion-approximation result for the solution of an ordinary differential equation with random coefficients. This result is an extension of that stated in [24] to the case of processes with values in a Hilbert space. 
TheOREm 5.4. $\forall L>0$ and $\forall y \in \mathcal{H}_{\xi}^{\omega}=\mathbb{C}^{N(\omega)} \times L^{2}\left(\xi, k^{2}(\omega)\right)$, the family $\left(\mathbf{T}^{\xi, \epsilon}(\omega, .)(y)\right)_{\epsilon \in(0,1)}$, solutions of the differential equation (5.3), converges in distribution in $\mathcal{C}\left([0, L], \mathcal{H}_{\xi, w}^{\omega}\right)$ as $\epsilon \rightarrow 0$ to a limit denoted by $\mathbf{T}^{\xi}(\omega,).(y)$. Here $\mathcal{H}_{\xi, w}^{\omega}$ stands for the Hilbert space $\mathcal{H}_{\xi}^{\omega}$ equipped with the weak topology. This limit is the unique diffusion process on $\mathcal{H}_{\xi}^{\omega}$, starting from $y$, associated to the infinitesimal generator

$$
\mathcal{L}_{\xi}^{\omega}=\mathcal{L}_{1}^{\omega}+\mathcal{L}_{2, \xi}^{\omega}+\mathcal{L}_{3, \xi}^{\omega}
$$

where

$$
\begin{aligned}
\mathcal{L}_{1}^{\omega}= & \frac{1}{2} \sum_{\substack{j, l=1 \\
j \neq l}}^{N(\omega)} \Gamma_{j l}^{c}(\omega)\left(T_{j} \overline{T_{j}} \partial_{T_{l}} \partial_{\overline{T_{l}}}+T_{l} \overline{T_{l}} \partial_{T_{j}} \partial_{\overline{T_{j}}}-T_{j} T_{l} \partial_{T_{j}} \partial_{T_{l}}-\overline{T_{j} T_{l}} \partial_{\overline{T_{j}}} \partial_{\overline{T_{l}}}\right) \\
& +\frac{1}{2} \sum_{j, l=1}^{N(\omega)} \Gamma_{j l}^{1}(\omega)\left(T_{j} \overline{T_{l}} \partial_{T_{j}} \partial_{\overline{T_{l}}}+\overline{T_{j}} T_{l} \partial_{\overline{T_{j}}} \partial_{T_{l}}-T_{j} T_{l} \partial_{T_{j}} \partial_{T_{l}}-\overline{T_{j} T_{l}} \partial_{\overline{T_{j}}} \partial_{\overline{T_{l}}}\right) \\
& +\frac{1}{2} \sum_{j=1}^{N(\omega)}\left(\Gamma_{j j}^{c}(\omega)-\Gamma_{j j}^{1}(\omega)\right)\left(T_{j} \partial_{T_{j}}+\overline{T_{j}} \partial_{\overline{T_{j}}}\right)+\frac{i}{2} \sum_{j=1}^{N(\omega)} \Gamma_{j j}^{s}(\omega)\left(T_{j} \partial_{T_{j}}-\overline{T_{j}} \partial_{\overline{T_{j}}}\right),
\end{aligned}
$$

and

$$
\begin{aligned}
& \mathcal{L}_{2, \xi}^{\omega}=-\frac{1}{2} \sum_{j=1}^{N(\omega)}\left(\Lambda_{j}^{c, \xi}(\omega)+i \Lambda_{j}^{s, \xi}(\omega)\right) T_{j} \partial_{T_{j}}+\left(\Lambda_{j}^{c, \xi}(\omega)-i \Lambda_{j}^{s, \xi}(\omega)\right) \overline{T_{j}} \partial_{\overline{T_{j}}}, \\
& \mathcal{L}_{3, \xi}^{\omega}=i \sum_{j=1}^{N(\omega)} \kappa_{j}^{\xi}(\omega)\left(T_{j} \partial_{T_{j}}-\overline{T_{j}} \partial_{\overline{T_{j}}}\right) .
\end{aligned}
$$

Here, we have considered the classical complex derivative with the following notation: If $v=v_{1}+i v_{2}$, then $\partial_{v}=\frac{1}{2}\left(\partial_{v_{1}}-i \partial_{v_{2}}\right)$ and $\partial_{\bar{v}}=\frac{1}{2}\left(\partial_{v_{1}}+i \partial_{v_{2}}\right)$. We have used the following notations: $\forall(j, l) \in\{1, \ldots, N(\omega)\}^{2}$ and $j \neq l$,

$$
\begin{aligned}
\Gamma_{j l}^{c}(\omega) & =\frac{k^{4}(\omega)}{2 \beta_{j}(\omega) \beta_{l}(\omega)} \int_{0}^{+\infty} \mathbb{E}\left[C_{j l}^{\omega}(0) C_{j l}^{\omega}(z)\right] \cos \left(\left(\beta_{l}(\omega)-\beta_{j}(\omega)\right) z\right) d z, \\
\Gamma_{j j}^{c}(\omega) & =-\sum_{\substack{l=1 \\
l \neq j}}^{N(\omega)} \Gamma_{j l}^{c}(\omega), \\
\Gamma_{j l}^{s}(\omega) & =\frac{k^{4}(\omega)}{2 \beta_{j}(\omega) \beta_{l}(\omega)} \int_{0}^{+\infty} \mathbb{E}\left[C_{j l}^{\omega}(0) C_{j l}^{\omega}(z)\right] \sin \left(\left(\beta_{l}(\omega)-\beta_{j}(\omega)\right) z\right) d z, \\
\Gamma_{j j}^{s}(\omega) & =-\sum_{\substack{l=1 \\
l \neq j}}^{N(\omega)} \Gamma_{j l}^{s}(\omega),
\end{aligned}
$$

and $\forall(j, l) \in\{1, \ldots, N(\omega)\}^{2}$, 


$$
\begin{aligned}
\Gamma_{j l}^{1}(\omega) & =\frac{k^{4}(\omega)}{2 \beta_{j}(\omega) \beta_{l}(\omega)} \int_{0}^{+\infty} \mathbb{E}\left[C_{j j}^{\omega}(0) C_{l l}^{\omega}(z)\right] d z \\
\Lambda_{j}^{c, \xi}(\omega) & =\int_{\xi}^{k^{2}(\omega)} \frac{k^{4}(\omega)}{2 \sqrt{\gamma^{\prime}} \beta_{j}(\omega)} \int_{0}^{+\infty} \mathbb{E}\left[C_{j \gamma^{\prime}}^{\omega}(0) C_{j \gamma^{\prime}}^{\omega}(z)\right] \cos \left(\left(\sqrt{\gamma^{\prime}}-\beta_{j}(\omega)\right) z\right) d z d \gamma^{\prime}, \\
\Lambda_{j}^{s, \xi}(\omega) & =\int_{\xi}^{k^{2}(\omega)} \frac{k^{4}(\omega)}{2 \sqrt{\gamma^{\prime}} \beta_{j}(\omega)} \int_{0}^{+\infty} \mathbb{E}\left[C_{j \gamma^{\prime}}^{\omega}(0) C_{j \gamma^{\prime}}^{\omega}(z)\right] \sin \left(\left(\sqrt{\gamma^{\prime}}-\beta_{j}(\omega)\right) z\right) d z d \gamma^{\prime}, \\
\kappa_{j}^{\xi}(\omega) & =\int_{-\infty}^{-\xi} \frac{k^{4}(\omega)}{2 \beta_{j}(\omega) \sqrt{\left|\gamma^{\prime}\right|}} \int_{0}^{+\infty} \mathbb{E}\left[C_{j \gamma^{\prime}}^{\omega}(0) C_{j \gamma^{\prime}}^{\omega}(z)\right] \cos \left(\beta_{j}(\omega) z\right) e^{-\sqrt{\left|\gamma^{\prime}\right| z} d z d \gamma^{\prime}}
\end{aligned}
$$

The coupling coefficients $C^{\omega}(z)$ are defined by (4.2). We obtain the following result in the asymptotic $\xi \rightarrow 0$.

TheOREM 5.5. $\forall L>0$ and $\forall y \in \mathcal{H}_{0}^{\omega}=\mathbb{C}^{N(\omega)} \times L^{2}\left(0, k^{2}(\omega)\right)$, the family $\left(\mathbf{T}^{\xi}(\omega, .)(y)\right)_{\xi \in(0,1)}$ converges in distribution in $\mathcal{C}\left([0, L],\left(\mathcal{H}_{0}^{\omega},\|\cdot\|_{\mathcal{H}_{0}^{\omega}}\right)\right)$ as $\xi \rightarrow 0$ to a limit denoted by $\mathbf{T}^{0}(\omega,).(y)$. This limit is the unique diffusion process on $\mathcal{H}_{0}^{\omega}$, starting from $y$, associated to the infinitesimal generator

$$
\mathcal{L}^{\omega}=\mathcal{L}_{1}^{\omega}+\mathcal{L}_{2}^{\omega}+\mathcal{L}_{3}^{\omega}
$$

where

$$
\begin{aligned}
& \mathcal{L}_{2}^{\omega}=-\frac{1}{2} \sum_{j=1}^{N(\omega)}\left(\Lambda_{j}^{c}(\omega)+i \Lambda_{j}^{s}(\omega)\right) T_{j} \partial_{T_{j}}+\left(\Lambda_{j}^{c}(\omega)-i \Lambda_{j}^{s}(\omega)\right) \overline{T_{j}} \partial_{\overline{T_{j}}} \\
& \mathcal{L}_{3}^{\omega}=i \sum_{j=1}^{N(\omega)} \kappa_{j}(\omega)\left(T_{j} \partial_{T_{j}}-\overline{T_{j}} \partial_{\overline{T_{j}}}\right) .
\end{aligned}
$$

Here, we have, $\forall j \in\{1, \ldots, N(\omega)\}$,

$$
\Lambda_{j}^{c}(\omega)=\lim _{\xi \rightarrow 0} \Lambda_{j}^{c, \xi}(\omega), \quad \Lambda_{j}^{s}(\omega)=\lim _{\xi \rightarrow 0} \Lambda_{j}^{s, \xi}(\omega), \quad \text { and } \quad \kappa_{j}(\omega)=\lim _{\xi \rightarrow 0} \kappa_{j}^{\xi}(\omega) .
$$

Theorem 5.4 and 5.5 describe the asymptotic behavior, first as $\epsilon \rightarrow 0$ and second as $\xi \rightarrow 0$, of the statistical properties of the transfer operator $\mathbf{T}^{\xi, \epsilon}(\omega, L)$ in terms of a diffusion process. In the appendix we give the sketches of proofs of Theorem 5.4 and 5.5. They are based on a martingale approach using the perturbed-test-function method. In a first step we show the tightness of the processes, and in a second step we characterize all subsequence limits by means of a well-posed martingale problem in a Hilbert space. However, the full proofs of Theorem 5.4 and 5.5 are given in [12].

The infinitesimal generator $\mathcal{L}^{\omega}$ is composed of three parts which represent different behaviors on the diffusion process, and it involves only the $N(\omega)$-discrete coordinates. The first operator $\mathcal{L}_{1}^{\omega}$ describes the coupling between the $N(\omega)$-propagating modes. This part is of the form of the infinitesimal generator obtained in $[7,9]$, and the total energy is conserved. The second operator $\mathcal{L}_{2}^{\omega}$ describes the coupling between the propagating modes with the radiating modes. This part implies a mode-dependent and frequency-dependent attenuation on the $N(\omega)$-propagating modes that we study in Section 6.1, and a mode-dependent and frequency-dependent phase modulation. The 
third operator $\mathcal{L}_{3}^{\omega}$ describes the coupling between the propagating and the evanescent modes, and implies a mode-dependent and frequency-dependent phase modulation. The purely imaginary part of the operator $\mathcal{L}^{\omega}$ does not remove energy from the propagating modes but gives an effective dispersion.

Moreover, because the infinitesimal generator $\mathcal{L}^{\omega}$ involves only the $N(\omega)$-discrete coordinate, the amplitude of the radiating modes remain constant on $L^{2}\left(0, k^{2}(\omega)\right)$ and the statistical properties of the propagating and the radiating modes are independent. From this decoupling it is not possible for the random fluctuations of the medium to cause energy transfer form the radiating modes to the propagating modes. Consequently, the propagating modes can lose energy into the radiating modes and get lost into the bottom of the ocean as we will see in Section 6.1.

Let us remark that the convergence in Theorem 5.4 holds also on $\mathcal{C}\left([0, L],\left(\mathcal{H}_{\xi}^{\omega},\|\cdot\|_{\mathcal{H}_{\xi}}\right)\right)$ for the $N(\omega)$-discrete propagating mode amplitudes.

5.2. Mean mode amplitudes. In this section we study the asymptotic mean mode amplitudes. From Theorem 5.5, we get the following result about the mean mode amplitudes.

Proposition 5.6. $\forall y \in \mathcal{H}_{0}^{\omega}, \forall z \in[0, L]$, and $\forall j \in\{1, \ldots, N(\omega)\}$,

$$
\begin{aligned}
\lim _{\xi \rightarrow 0 \epsilon \rightarrow 0} \lim _{\epsilon \rightarrow} \mathbb{E} & {\left[\mathbf{T}_{j}^{\xi, \epsilon}(\omega, z)(y)\right]=\mathbb{E}\left[\mathbf{T}_{j}^{0}(\omega, z)(y)\right] } \\
& =\exp \left[\left(\frac{\Gamma_{j j}^{c}(\omega)-\Gamma_{j j}^{1}(\omega)-\Lambda_{j}^{c}(\omega)}{2}\right) z+i\left(\frac{\Gamma_{j j}^{s}(\omega)-\Lambda_{j j}^{s}(\omega)}{2}+k_{j}(\omega)\right) z\right] y_{j}(\omega) .
\end{aligned}
$$

First, let us recall that the mean amplitude of the radiating part remains constant on $L^{2}\left(0, k^{2}(\omega)\right)$. Second, $\forall j \in\{1, \ldots, N(\omega)\}$, the coefficient $\left(\Gamma_{j j}^{1}(\omega)+\Lambda_{j}^{c}(\omega)-\Gamma_{j j}^{c}(\omega)\right) / 2$ is nonnegative. In fact, for $(j, l) \in\{1, \ldots, N(\omega)\}^{2}$ such that $j \neq l, \Gamma_{j l}^{c}(\omega)$ and $\Gamma_{j j}^{1}(\omega)$ are nonnegative because they are proportional to the power spectral density of $C_{j l}^{\omega}$ and $C_{j j}^{\omega}$ at $\beta_{l}(\omega)-\beta_{j}(\omega)$ and 0 wavenumbers. Therefore, $-\Gamma_{j j}^{c}(\omega)$ is also nonnegative. Moreover, $\Lambda_{j}^{c}(\omega)$ is also nonnegative because it is proportional to the integral over $\left(0, k^{2}(\omega)\right)$ of the power spectral density of $C_{j \gamma}^{\omega}$ at $\sqrt{\gamma}-\beta_{j}(\omega)$ wavenumber.

The exponential decay rate for the mean $j$ th-propagating mode is given by

$$
\left|\mathbb{E}\left[\mathbf{T}_{j}^{0}(\omega, L)(y)\right]\right|=\left|y_{j}\right| \exp \left[-\left(\frac{\Gamma_{j j}^{1}(\omega)-\Gamma_{j j}^{c}(\omega)+\Lambda_{j}^{c}(\omega)}{2}\right) L\right],
$$

which depends on the effective coupling between the propagating modes, and the coupling between the propagating and the radiating modes. This exponential decay corresponds to a loss of coherence of the transmitted field.

\section{Coupled power equations}

This section is devoted to the analysis of the asymptotic mean mode powers of the propagating modes. More precisely, we study the asymptotic effects of the coupling between the propagating modes and the radiating modes. Let

$$
\mathcal{T}_{j}^{l}(\omega, z)=\lim _{\xi \rightarrow 0 \in \rightarrow 0} \lim _{\epsilon \rightarrow} \mathbb{E}\left[\left|\mathbf{T}_{j}^{\xi, \epsilon}(\omega, L)\left(y^{l}\right)\right|^{2}\right]=\mathbb{E}\left[\left|\mathbf{T}_{j}^{0}(\omega, z)\left(y^{l}\right)\right|^{2}\right]
$$

be the asymptotic mean mode power of the $j$ th propagating modes. $\mathcal{T}_{j}^{l}(\omega, L)$ is the expected power of the $j$ th propagating mode at the propagation distance $z=L$. 


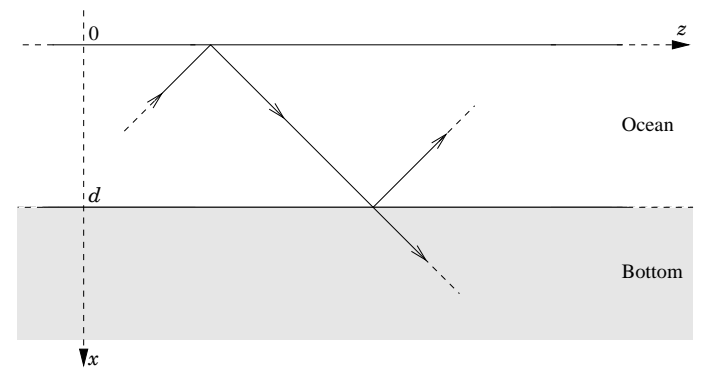

FIG. 6.1. Illustration of the radiative loss in the shallow-water random waveguide model.

Here $y^{l} \in \mathcal{H}_{0}^{\omega}$ is defined by $y_{j}^{l}=\delta_{j l}$ and $y_{\gamma}^{l}=0$ for $\gamma \in\left(0, k^{2}(\omega)\right)$, and where $\delta_{j l}$ is the Kronecker symbol. The initial condition $y^{l}$ means that an impulse equal to one charges only the $l$ th propagating mode. From Theorem 5.5, we have the coupled power equations:

$$
\frac{d}{d z} \mathcal{T}_{j}^{l}(\omega, z)=-\Lambda_{j}^{c}(\omega) \mathcal{T}_{j}^{l}(\omega, z)+\sum_{\substack{n=1 \\ n \neq j}}^{N(\omega)} \Gamma_{n j}^{c}(\omega)\left(\mathcal{T}_{n}^{l}(\omega, z)-\mathcal{T}_{j}^{l}(\omega, z)\right),
$$

with initial conditions $\mathcal{T}_{j}^{l}(\omega, 0)=\delta_{j l}$. These equations describe the transfer of energy between the propagating modes and $\Gamma^{c}(\omega)$ is the energy transport matrix. In our context, we also have the coefficients $\Lambda_{j}^{c}(\omega)$ given by the coupling between the propagating modes and the radiating modes. These coefficients, defined in Theorem 5.5, are responsible for the radiative loss of energy in the ocean bottom (see Figure 6.1). This loss of energy is described more precisely in the following section.

6.1. Exponential decay of the propagating modes energy. In this section, we assume that at least one of the coefficients $\Lambda^{c}(\omega)$ is positive. With this assumption, we show that the total energy carried by the propagating modes decays exponentially with the size $L$ of the random section. In the opposite situation, that is when there is no radiative loss $\Lambda^{c}(\omega)=0$, it has been shown in [7] and [9, Chapter 20] that the energy of the propagating modes is conserved and for large $L$ the asymptotic distribution of the energy becomes uniform over the propagating modes.

Let us define

$$
\mathcal{S}_{+}^{N(\omega)}=\left\{X \in \mathbb{R}^{N(\omega)}, X_{j} \geq 0 \quad \forall j \in\{1, \ldots, N(\omega)\} \text { and }\|X\|_{2, \mathbb{R}^{N(\omega)}}^{2}=\langle X, X\rangle_{\mathbb{R}^{N(\omega)}}=1\right\}
$$

with $\langle X, Y\rangle_{\mathbb{R}^{N(\omega)}}=\sum_{j=1}^{N(\omega)} X_{j} Y_{j}$ for $(X, Y) \in\left(\mathbb{R}^{N(\omega)}\right)^{2}$, and

$$
\Lambda_{d}^{c}(\omega)=\operatorname{diag}\left(\Lambda_{1}^{c}(\omega), \ldots, \Lambda_{N(\omega)}^{c}(\omega)\right) .
$$


THEOREM 6.1. Let us assume that the energy transport matrix $\Gamma^{c}(\omega)$ is irreducible. Then we have

$$
\lim _{L \rightarrow+\infty} \frac{1}{L} \ln \left[\sum_{j=1}^{N(\omega)} \mathcal{T}_{j}^{l}(\omega, L)\right]=-\Lambda_{\infty}(\omega)
$$

with

$$
\Lambda_{\infty}(\omega)=\inf _{X \in \mathcal{S}_{+}^{N(\omega)}}\left\langle\left(-\Gamma^{c}(\omega)+\Lambda_{d}^{c}(\omega)\right) X, X\right\rangle_{\mathbb{R}^{N(\omega)}},
$$

which is positive as soon as one of the coefficients $\Lambda_{j}^{c}(\omega)$ is positive.

This result means that the expected powers of the propagating modes decays exponentially with the propagation distance, and the decay rate can be expressed in terms of a variational formula over a finite-dimensional space.

Proof. The coupled power equations admit a probabilistic representation in terms of a jump Markov process. If we denote by $\left(Y_{t}^{N(\omega)}\right)_{t \geq 0}$ a jump Markov process with state space $\{1, \ldots, N(\omega)\}$ and intensity matrix $\Gamma^{c}(\omega)$, then we have, using the Feynman-Kac formula,

$$
\mathcal{T}_{j}^{l}(\omega, z)=\mathbb{E}\left[\exp \left(-\int_{0}^{z} \Lambda_{Y_{s}^{N(\omega)}}^{c}(\omega) d s\right) \mathbf{1}_{\left(Y_{z}^{N(\omega)}=j\right)} \mid Y_{0}^{N(\omega)}=l\right] .
$$

Moreover, we have supposed that $\Gamma^{c}(\omega)$ is irreducible. Then $\left(Y_{t}^{N(\omega)}\right)_{t \geq 0}$ in an ergodic process with invariant measure $\mu_{N(\omega)}$, which is the uniform distribution over $\{1, \ldots, N(\omega)\}$. That is, $\mu_{N(\omega)}(j)=1 / N(\omega) \forall j \in\{1, \ldots, N(\omega)\}$. Let us consider the local time

$$
l_{T}(j)=\int_{0}^{T} \mathbf{1}_{\left(Y_{s}^{N(\omega)}=j\right)} d s
$$

for $j \in\{1, \ldots, N(\omega)\}$ and $T>0$, which corresponds to the time spent by the process $\left(Y_{t}^{N(\omega)}\right)_{t \geq 0}$ in the state $j$ during the time interval $[0, T]$. According to [5], we have a large deviation principle for $\frac{1}{T} l_{T}$ viewed as a random process with values in $\mathcal{M}_{1}^{N(\omega)}$, which is the set of probability measures on $\{1, \ldots, N(\omega)\}$. More precisely, we have

$$
\begin{aligned}
\lim _{L \rightarrow+\infty} \frac{1}{L} \ln \mathbb{E}\left[\exp \left(-L\left\langle\Lambda^{c}, \frac{1}{L} l_{L}\right\rangle_{\mathbb{R}^{N(\omega)}}\right) \mid Y_{0}^{N(\omega)}=l\right] & \\
& =\lim _{L \rightarrow+\infty} \frac{1}{L} \ln \mathbb{E}\left[\exp \left(-\int_{0}^{L} \Lambda_{Y_{s}^{N(\omega)}}^{c} d s\right) \mid Y_{0}^{N(\omega)}=l\right] \\
& =-\inf _{\mu \in \mathcal{M}_{1}^{N(\omega)}}\left(I(\mu)+\left\langle\Lambda^{c}(\omega), \mu\right\rangle\right),
\end{aligned}
$$

with

$$
I(\mu)=\left\|\left(-\Gamma^{c}(\omega)\right)^{1 / 2} \sqrt{\mu}\right\|_{2, \mathbb{R}^{N(\omega)}}^{2}=\left\langle\left(-\Gamma^{c}(\omega)\right) \sqrt{\mu}, \sqrt{\mu}\right\rangle_{\mathbb{R}^{N(\omega)}} .
$$

Consequently,

$$
\lim _{L \rightarrow+\infty} \frac{1}{L} \ln \left[\sum_{j=1}^{N(\omega)} \mathcal{T}_{j}^{l}(\omega, L)\right]=-\Lambda_{\infty}(\omega)
$$


Finally, using the compactness of $\mathcal{S}_{+}^{N(\omega)}, \Lambda_{\infty}(\omega)$ is positive because $-\Gamma^{c}(\omega)$ and $\Lambda_{d}^{c}(\omega)$ are two nonnegative matrices, and 0 is a simple eigenvalue of $-\Gamma^{c}(\omega)$ by the PerronFrobenius theorem.

The expression (6.3) of $\Lambda_{\infty}(\omega)$ is not simple. However, we have the following inequalities:

$$
\min _{j \in\{1, \ldots, N(\omega)\}} \Lambda_{j}^{c}(\omega) \leq \Lambda_{\infty}(\omega) \leq \bar{\Lambda}(\omega)=\frac{1}{N(\omega)} \sum_{j=1}^{N(\omega)} \Lambda_{j}^{c}(\omega) .
$$

To finish this section, let us investigate some special cases in which we can give a simple expression of $\Lambda_{\infty}(\omega)$. $(6.5)$,

First, we assume that $\forall j \in\{1, \ldots, N(\omega)\}, \Lambda_{j}^{c}(\omega)=\Lambda(\omega)>0$. In this case, using

$$
\Lambda_{\infty}(\omega)=\Lambda(\omega)
$$

This means that if all the coefficients which represent the radiation losses are equal, the decay rate of the total energy of the propagating modes is given by this coefficient.

Second, we assume that the coupling matrix is small, that is, we replace $\Gamma^{c}(\omega)$ by $\tau \Gamma^{c}(\omega)$ with $\tau \ll 1$. If $\forall j \in\{1, \ldots, N(\omega)\} \Lambda_{j}^{c}(\omega)>0$, we have

$$
\lim _{\tau \rightarrow 0} \Lambda_{\infty}^{\tau}(\omega)=\min _{j \in\{1, \ldots, N(\omega)\}} \Lambda_{j}^{c}(\omega) .
$$

From (6.5), this is the smallest value that $\Lambda_{\infty}(\omega)$ can take. This result is consistent with the fact that the coupling process on the transfer of energy between propagating modes is negligible and the decay rate of the energy of a particular propagating mode $j$ is given by its own decay coefficient $\Lambda_{j}(\omega)$. Then, for the total energy of propagating modes the decay rate is given by the minimum of those decay coefficients. Consequently, if there exists $\Lambda_{j_{0}}^{c}(\omega)=0$, we have

$$
\lim _{\tau \rightarrow 0} \Lambda_{\infty}^{\tau}(\omega)=0
$$

The reason is that the energy of the $j_{0}$ th propagating mode stays approximately constant with a weak transfer of energy, and

$$
\lim _{\tau \rightarrow 0} \frac{1}{\tau} \Lambda_{\infty}^{\tau}(\omega)=\inf _{X \in \tilde{V}}\left\langle\left(-\Gamma^{c}(\omega)\right) X, X\right\rangle_{\mathbb{R}^{N(\omega)}}>0
$$

where $\tilde{V}=\left\{X \in \mathcal{S}_{+}^{N(\omega)}, \quad \operatorname{supp} X \subset\{1, \ldots, N(\omega)\} \backslash \operatorname{supp}\left(\Lambda^{c}(\omega)\right)\right\}$, because $\sqrt{\mu_{N(\omega)}} \notin \tilde{V}$.

Now, we assume that the coupling matrix is large, that is we replace $\Gamma^{c}(\omega)$ by $\frac{1}{\tau} \Gamma^{c}(\omega)$ with $\tau \ll 1$. In this case, we have

$$
\lim _{\tau \rightarrow 0} \Lambda_{\infty}^{\tau}(\omega)=\bar{\Lambda}(\omega)
$$

From (6.5), this is the largest value that $\Lambda_{\infty}(\omega)$ can take. The strong coupling produces a uniform distribution of energy over the propagating modes and the decay 


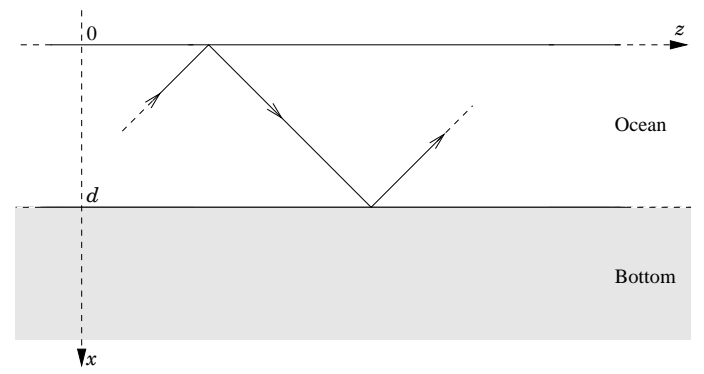

FIG. 6.2. Illustration of negligible radiation losses in the shallow-water random waveguide model.

rate becomes $\left\langle\Lambda^{c}(\omega), \mu_{N(\omega)}\right\rangle_{\mathbb{R}^{N(\omega)}}=\bar{\Lambda}(\omega)$ for each mode. A more convenient way to get this result is to use a probabilistic representation. In fact, we have

$$
\begin{aligned}
\mathcal{T}_{j}^{l}(\omega, z) & =\mathbb{E}\left[\exp \left(-\int_{0}^{z} \Lambda_{Y_{s / \tau}^{N(\omega)}}^{c}(\omega)\right) \mathbf{1}_{\left(Y_{z}^{N(\omega)}=j\right)} \mid Y_{0}^{N(\omega)}=l\right] \\
& =\mathbb{E}\left[\exp \left(-z \frac{\tau}{z} \int_{0}^{z / \tau} \Lambda_{Y_{s}^{N(\omega)}}^{c}(\omega)\right) \mathbf{1}_{\left(Y_{z / \tau}^{N(\omega)}=j\right)} \mid Y_{0}^{N(\omega)}=l\right],
\end{aligned}
$$

where $\left(Y_{t}^{N(\omega)}\right)_{t \geq 0}$ is a jump Markov process with state space $\{1, \ldots, N(\omega)\}$ and intensity matrix $\Gamma^{c}(\omega)$. Using the ergodic properties of $\left(Y_{t}^{N(\omega)}\right)_{t \geq 0}$, we get that

$$
\lim _{\tau \rightarrow 0} \mathcal{T}_{j}^{\tau, l}(\omega, L)=\frac{1}{N(\omega)} \exp (-\bar{\Lambda}(\omega) L) .
$$

Finally, if we assume that the radiation losses are negligible, that is we replace $\Lambda^{c}(\omega)$ by $\tau \Lambda^{c}(\omega)$ with $\tau \ll 1$, we have

$$
\lim _{\tau \rightarrow 0} \Lambda_{\infty}^{\tau}(\omega)=0 .
$$

In fact, if the radiative loss is negligible, the coupling process becomes dominant, and we can show that

$$
\forall L>0, \quad \sup _{z \in[0, L]}\left\|\mathcal{T}_{j}^{\tau, l}(\omega, z)-\mathcal{T}_{j}^{0, l}(\omega, z)\right\|_{2, \mathbb{R}^{N(\omega)}}=\mathcal{O}(\tau),
$$

where $\mathcal{T}^{0, l}(\omega,$.$) satisfies (6.2)$ without the coefficient $\Lambda^{c}(\omega)$. In this situation

$$
\mathcal{T}_{j}^{0, l}(\omega, L)=\mathbb{P}\left(Y_{L}^{N(\omega)}=j \mid Y_{0}^{N(\omega)}=l\right),
$$

and the total energy is conserved (see Figure 6.2), and

$$
\lim _{\tau \rightarrow 0} \frac{1}{\tau} \Lambda_{\infty}^{\tau}(\omega)=\bar{\Lambda}(\omega)>0
$$

As it was already observed in [9] the modal energy distribution converges as $L \rightarrow+\infty$ to a uniform distribution:

$$
\lim _{L \rightarrow+\infty} \mathcal{T}_{j}^{0, l}(\omega, L)=\frac{1}{N(\omega)} .
$$


6.2. Continuum approximation to coupled power equations. In this section, under the assumption that the nearest-neighbor coupling introduced in Section 6.2.1 is the main power transfer mechanism, we give an approximate solution of the coupled power equations (6.2) in the limit of a large number of propagating modes $N(\omega) \gg 1$. Let us note that the limit of a large number of propagating modes $N(\omega) \gg 1$ corresponds to the regime $\omega d \gg 1$. Next, we analyze the energy carried by the propagating modes in this regime.

The coupled power equations can be approximated in the limit of a large number of propagating modes $N(\omega) \gg 1$ by a diffusion equation. This approximation has already been obtained in [15] for instance, in which we can find further references about this topic. We can also refer to [20] for more discussions on this approximation. For an application of such a diffusion model to acoustic propagation in random sound channels we refer to [21], and for applications to time reversal of waves we refer to [11].

Using the form of the covariance function (2.4), we find

$$
\Gamma_{j l}^{c}(\omega)=\frac{a k^{4}(\omega) I_{j, l}(\omega)}{2 \beta_{j}(\omega) \beta_{l}(\omega)\left(a^{2}+\left(\beta_{j}(\omega)-\beta_{l}(\omega)\right)^{2}\right)}
$$

and

$$
\Lambda_{j}^{c}(\omega)=\int_{0}^{k^{2}(\omega)} \frac{a k^{4}(\omega) I_{j, \gamma}(\omega)}{2 \beta_{j}(\omega) \sqrt{\gamma}\left(a^{2}+\left(\beta_{j}(\omega)-\sqrt{\gamma}\right)^{2}\right)} d \gamma
$$

where

$$
\begin{aligned}
I_{j l}=\frac{1}{4} A_{j}^{2} A_{l}^{2}[ & S\left(\sigma_{j}-\sigma_{l}, \sigma_{j}-\sigma_{l}\right)+S\left(\sigma_{j}+\sigma_{l}, \sigma_{j}+\sigma_{l}\right) \\
- & \left.S\left(\sigma_{j}-\sigma_{l}, \sigma_{j}+\sigma_{l}\right)-S\left(\sigma_{j}+\sigma_{l}, \sigma_{j}-\sigma_{l}\right)\right], \\
I_{j \gamma}=\frac{1}{4} A_{j}^{2} A_{\gamma}^{2}[ & S\left(\sigma_{j}-\eta, \sigma_{j}-\eta\right)+S\left(\sigma_{j}+\eta, \sigma_{j}+\eta\right) \\
- & \left.S\left(\sigma_{j}-\eta, \sigma_{j}+\eta\right)-S\left(\sigma_{j}+\eta, \sigma_{j}-\eta\right)\right],
\end{aligned}
$$

with $S\left(v_{1}, v_{2}\right)=\int_{0}^{d} \int_{0}^{d} \gamma_{0}\left(x_{1}, x_{2}\right) \cos \left(\frac{v_{1}}{d} x_{1}\right) \cos \left(\frac{v_{2}}{d} x_{2}\right) d x_{1} d x_{2}$, and where $A_{j}(\omega), A_{\gamma}(\omega)$, $\sigma_{j}(\omega), \eta(\omega), \phi_{j}(\omega, x)$, and $\phi_{\gamma}(\omega, x)$ are defined in Section 3.1.

6.2.1. Band-limiting idealization. In this section, we introduce a bandlimiting idealization hypothesis in which the power spectral density of the random fluctuations is assumed to be limited in both the transverse and the longitudinal directions. We assume that the support of $S$ lies in the square $\left[-\frac{3 \pi}{2}, \frac{3 \pi}{2}\right] \times\left[-\frac{3 \pi}{2}, \frac{3 \pi}{2}\right]$. Then,

$$
I_{j l}(\omega)=\left\{\begin{array}{cl}
\frac{1}{4} A_{j}^{2}(\omega) A_{l}^{2}(\omega) S\left(\sigma_{j}(\omega)-\sigma_{l}(\omega), \sigma_{j}(\omega)-\sigma_{l}(\omega)\right), & \text { if }|j-l|=1, \\
0, & \text { otherwise },
\end{array}\right.
$$

and

$$
I_{j \gamma}(\omega)=\left\{\begin{array}{cl}
\frac{1}{4} A_{j}^{2}(\omega) A_{l}^{2}(\omega) S\left(\sigma_{j}(\omega)-\eta(\omega), \sigma_{j}(\omega)-\eta(\omega)\right), & \text { if }\left|\sigma_{j}(\omega)-\eta(\omega)\right| \leq \frac{3 \pi}{2} \\
0, & \text { otherwise }
\end{array}\right.
$$


From this assumption we obtain $\forall 0<\gamma<k^{2}(\omega)$ and $j \in\{1, \ldots, N(\omega)-2\}$,

$$
\eta(\omega)-\sigma_{j}(\omega) \geq n_{1} k(\omega) d \sqrt{1-\frac{1}{n_{1}^{2}}}-\sigma_{j}(\omega) \pi \underbrace{\left(\frac{n_{1} k(\omega) d}{\pi} \theta-N(\omega)\right)}_{\in[0,1)}+2 \pi .
$$

Then, for $j \in\{1, \ldots, N(\omega)-2\}$,

$$
\inf _{0<\gamma<k^{2}} \eta(\omega)-\sigma_{j}(\omega)>\frac{3 \pi}{2},
$$

and $\Lambda_{j}^{c}(\omega)=0, \forall j \in\{1, \ldots, N(\omega)-2\}$. Consequently, the coupled power equations (6.2) become

$$
\begin{aligned}
\frac{d}{d z} \mathcal{T}_{N}^{l}(z) & =-\Lambda_{N}^{c} \mathcal{T}_{N}^{l}(z)+\Gamma_{N-1 N}^{c}\left(\mathcal{T}_{N-1}^{l}(z)-\mathcal{T}_{N}^{l}(z)\right) \\
\frac{d}{d z} \mathcal{T}_{N-1}^{l}(z)=-\Lambda_{N-1}^{c} \mathcal{T}_{N-1}^{l}(z)+ & \Gamma_{N-1 N-2}^{c}\left(\mathcal{T}_{N-2}^{l}(z)-\mathcal{T}_{N-1}^{l}(z)\right) \\
& +\Gamma_{N-1 N}^{c}\left(\mathcal{T}_{N}^{l}(z)-\mathcal{T}_{N-1}^{l}(z)\right) \\
\frac{d}{d z} \mathcal{T}_{j}^{l}(z)= & \Gamma_{j-1 j}^{c}\left(\mathcal{T}_{j-1}^{l}(z)-\mathcal{T}_{j}^{l}(z)\right)+\Gamma_{j+1 j}^{c}\left(\mathcal{T}_{j+1}^{l}(z)-\mathcal{T}_{j}^{l}(z)\right) \text { for } j \in\{2, \ldots, N-2\} \\
\frac{d}{d z} \mathcal{T}_{1}^{l}(z)= & \Gamma_{21}^{c}\left(\mathcal{T}_{2}^{l}(z)-\mathcal{T}_{1}^{l}(z)\right)
\end{aligned}
$$

with $\mathcal{T}_{j}^{l}(0)=\delta_{j l}$.

The band-limiting idealization hypothesis is tantamount to a nearest-neighbor coupling. More precisely, this assumption implies that $\forall(j, l) \in\{1, \ldots, N(\omega)\}^{2}$, the $j$ th mode amplitude can exchange informations with the $l$ th amplitude mode if they are direct neighbors, that is, if they satisfy $|j-l| \leq 1$.

6.2.2. Continuum approximation. The evolution of the mean mode powers of the propagating modes can be described, in the limit of a large number of propagating modes $N(\omega) \gg 1$, by a diffusion model. This diffusive continuous model is equipped with boundary conditions which take into account the effect of the radiating modes at the bottom and the free surface of the waveguide (see Figure 6.1)

Let, $\forall \varphi \in \mathcal{C}^{0}([0,1]), \forall u \in[0,1]$, and $z \geq 0$,

$$
\mathcal{T}_{\varphi}^{N(\omega)}(z, u)=\mathcal{T}_{\varphi}^{[N(\omega) u]}(\omega, z)=\sum_{j=1}^{N} \varphi\left(\frac{j}{N(\omega)}\right) \mathcal{T}_{j}^{[N(\omega) u]}(\omega, z)
$$

where $\varphi \mapsto \mathcal{T}_{\varphi}^{N(\omega)}(z,$.$) can be extended to an operator from L^{2}(0,1)$ to itself. Here, $L^{2}(0,1)$ is equipped with the inner product defined as follows: $\forall(\varphi, \psi) \in L^{2}(0,1)^{2}$, $\langle\varphi, \psi\rangle_{L^{2}(0,1)}=\int_{0}^{1} \varphi(v) \psi(v) d v$.

Theorem 6.2. We have

1. $\forall \varphi \in L^{2}(0,1)$ and $\forall z \geq 0$,

$$
\lim _{N(\omega) \rightarrow+\infty} \mathcal{T}_{\varphi}^{N(\omega)}(z, u)=\mathcal{T}_{\varphi}(z, u) \quad \text { in } L^{2}(0,1)
$$


where $\mathcal{T}_{\varphi}(z, u)$ satisfies the partial differential equation : $\forall(z, u) \in(0,+\infty) \times$ $(0,1)$,

$$
\frac{\partial}{\partial z} \mathcal{T}_{\varphi}(z, u)=\frac{\partial}{\partial u}\left(a_{\infty}(\cdot) \frac{\partial}{\partial u} \mathcal{T}_{\varphi}\right)(z, u)
$$

with the boundary conditions: $\forall z>0$

$$
\frac{\partial}{\partial u} \mathcal{T}_{\varphi}(z, 0)=0, \quad \mathcal{T}_{\varphi}(z, 1)=0, \quad \text { and } \quad \mathcal{T}_{\varphi}(0, u)=\varphi(u)
$$

2. $\forall u \in[0,1], \forall z \geq 0$, and $\forall \varphi \in \mathcal{C}^{0}([0,1])$ such that $\varphi(1)=0$, we have

$$
\lim _{N(\omega) \rightarrow+\infty} \mathcal{T}_{\varphi}^{N(\omega)}(z, u)=\mathcal{T}_{\varphi}(z, u) .
$$

Here,

$$
a_{\infty}(u)=\frac{a_{0}}{1-\left(1-\frac{\pi^{2}}{a^{2} d^{2}}\right)(\theta u)^{2}},
$$

with $a_{0}=\frac{\pi^{2} S_{0}}{2 a n_{1}^{4} d^{4} \theta^{2}}, \theta=\sqrt{1-1 / n_{1}^{2}}, S_{0}=\int_{0}^{d} \int_{0}^{d} \gamma_{0}\left(x_{1}, x_{2}\right) \cos \left(\frac{\pi}{d} x_{1}\right) \cos \left(\frac{\pi}{d} x_{2}\right) d x_{1} d x_{2} . n_{1}$ is the index of refraction in the ocean section $[0, d], 1 / a$ is the correlation length of the random inhomogeneities in the longitudinal direction, and $\gamma_{0}$ is the covariance function of the random inhomogeneities in the transverse direction.

This theorem is a continuum approximation in the limit of a large number of propagating modes $N(\omega) \gg 1$. This approximation gives us, in the limit of a large number of propagating modes, a diffusion model for the transfer of energy between the $N(\omega)$-discrete propagating modes, with a reflecting boundary condition at $x=0$ (the top of the waveguide in Figure 2.1) and an absorbing boundary condition at $u=1$ (the bottom of the waveguide in Figure 2.1) which represents the radiative loss (see Figure 6.1).

The sketch of proof of Theorem 6.2 is given in Section A.3. This proof is based on a probabilistic representation of $\mathcal{T}_{j}^{l}(\omega, z)$ in terms of a jump Markov process, and on a martingale approach. The full proof of Theorem 6.2 is given in [12].

6.2.3. Exponential decay in the continuum approximation. In the limit of a large number of propagating modes $N(\omega) \gg 1$, we also observe that the energy carried by the continuum of propagating modes decays exponentially with the propagation distance. The exponential decay of the energy in the continuum approximation is given by the following result.

Theorem 6.3. $\forall \varphi \in L^{2}(0,1) \backslash\{0\}$ such that $\varphi \geq 0$, and $\forall u \in[0,1)$,

$$
\lim _{L \rightarrow+\infty} \frac{1}{L} \ln \left[\mathcal{T}_{\varphi}(L, u)\right]=-\Lambda_{\infty}
$$

where

$$
\Lambda_{\infty}=\inf _{\varphi \in \mathcal{D}} \int_{0}^{1} a_{\infty}(v) \varphi^{\prime}(v)^{2} d v>0
$$

and

$$
\mathcal{D}=\left\{\varphi \in \mathcal{C}^{\infty}([0,1]), \quad\|\varphi\|_{L^{2}(0,1)}=1, \quad \frac{\partial}{\partial v} \varphi(0)=0, \quad \varphi(1)=0\right\}
$$


This result means that the energy carried by each propagating mode decays exponentially with the propagation distance, and the decay rate can be expressed in terms of a variational formula. Consequently, the spatial inhomogeneities of the medium and the geometry of the shallow-water waveguide lead us to an exponential decay phenomenon caused by the radiative loss into the ocean bottom. domain

Proof. We can see that the operator $P_{\infty}=\frac{\partial}{\partial v}\left(a_{\infty}(\cdot) \frac{\partial}{\partial v}\right)$ on $L^{2}([0,1])$, with

$$
\mathcal{D}\left(P_{\infty}\right)=\left\{\varphi \in H^{2}(0,1), \quad \frac{\partial}{\partial v} \varphi(0)=0, \quad \varphi(1)=0\right\},
$$

is self-adjoint. $P_{\infty}$ has a compact resolvent $R_{\lambda}=\left(\lambda I d-P_{\infty}\right)^{-1}$ because $[0,1]$ is a compact set and then it has a point spectrum $\left(\lambda_{j}\right)_{j \geq 1}$ with eigenvectors denoted by $\left(\phi_{\infty, j}\right)_{j \geq 1}$. Moreover, all the eigenspaces are finite-dimensional subspaces of $\mathcal{D}\left(P_{\infty}\right)$ and $\forall \varphi \in \mathcal{D}\left(P_{\infty}\right) \backslash\{0\}$,

$$
\left\langle P_{\infty}(\varphi), \varphi\right\rangle_{L^{2}(0,1)}<0
$$

Let us organize the point spectrum in the nonincreasing way, $\cdots<\lambda_{2}<\lambda_{1}<0$. We have

$$
\mathcal{T}_{\varphi}(L, v)=\sum_{j \geq 1}\left\langle\varphi, \phi_{\infty, j}\right\rangle_{L^{2}(0,1)} e^{\lambda_{j} L} \phi_{\infty, j}(v)
$$

LEMma 6.4. $\lambda_{1}$ is a simple eigenvalue and one can choose $\phi_{\infty, 1}$ such that $\phi_{\infty, 1}(v)>0$ $\forall v \in[0,1)$.

Proof. This lemma is a consequence of the Krein-Rutman theorem, but not its strongest form [28]. Indeed, the set of nonnegative functions in $L^{2}(0,1)$ has an empty interior. However, using the smoothness of the eigenvectors, the proof also works in our case. The full proof of Lemma 6.4 is given in [12].

As a result, $\forall \varphi \in L^{2}(0,1) \backslash\{0\}$ such that $\varphi \geq 0, \forall v \in[0,1)$ we get

$$
\lim _{L \rightarrow+\infty} \frac{1}{L} \ln \left[\mathcal{T}_{\varphi}(L, v)\right]=\lambda_{1},
$$

and

$$
\lambda_{1}=\sup _{\substack{\varphi \in \mathcal{D}\left(P_{\infty}\right) \\\|\varphi\|_{L^{2}([0,1])}=1}}\left\langle P_{\infty}(\varphi), \varphi\right\rangle_{L^{2}([0,1])}=-\Lambda_{\infty}<0
$$

In Theorem 6.3, we take $\varphi \in L^{2}(0,1) \backslash\{0\}$ such that $\varphi \geq 0$, which can be considered as being the initial repartition of energy over the continuum of modes. However, the result of Theorem 6.3 is also valid for any $\varphi \in L^{2}(0,1) \backslash\{0\}$ such that $\left.\left\langle\varphi, \phi_{\infty, 1}\right\rangle_{L^{2}(0, d)}\right\rangle$ 0 .

6.3. Continuum approximation to coupled power equation with negligible radiation losses. In the case of negligible radiation losses, we also get a continuous diffusive model for the coupled power equations in the limit of a large number of propagating modes $N(\omega) \gg 1$. This diffusive continuous model is equipped 
with boundary conditions which take into account the negligible effect of the radiation losses at the bottom and the free surface of the waveguide (see Figure 6.2).

Now, let us assume that the radiation losses are negligible, that is, $\Lambda^{c}(\omega)=\tau \tilde{\Lambda}^{c}(\omega)$ with $\tau \ll 1$. We have already remarked that, if the radiation losses are negligible, then the coupling process is predominant and we have

$$
\forall L>0, \quad \sup _{z \in[0, L]}\left\|\mathcal{T}_{j}^{\tau, l}(\omega, z)-\mathcal{T}_{j}^{0, l}(\omega, z)\right\|_{2, \mathbb{R}^{N(\omega)}}=\mathcal{O}(\tau),
$$

where $\mathcal{T}^{0, l}(\omega,$.$) satisfies$

$$
\begin{aligned}
\frac{d}{d z} \mathcal{T}_{N}^{0, l}(z) & =\Gamma_{N-1 N}^{c}\left(\mathcal{T}_{N-1}^{0, l}(z)-\mathcal{T}_{N}^{0, l}(z)\right) \\
\frac{d}{d z} \mathcal{T}_{j}^{0, l}(z) & =\Gamma_{j-1 j}^{c}\left(\mathcal{T}_{j-1}^{0, l}(z)-\mathcal{T}_{j}^{0, l}(z)\right)+\Gamma_{j+1 j}^{c}\left(\mathcal{T}_{j+1}^{0, l}(z)-\mathcal{T}_{j}^{0, l}(z)\right)
\end{aligned}
$$

for $j \in\{2, \ldots, N-1\}$, and

$$
\frac{d}{d z} \mathcal{T}_{1}^{0, l}(z)=\Gamma_{21}^{c}\left(\mathcal{T}_{2}^{0, l}(z)-\mathcal{T}_{1}^{0, l}(z)\right)
$$

with $\mathcal{T}_{j}^{0, l}(0)=\delta_{j l}$.

6.3.1. Continuum approximation. Let, $\forall \varphi \in \mathcal{C}^{0}([0,1]), \forall u \in[0,1]$, and $z \geq 0$,

$$
\mathcal{T}_{\varphi}^{N(\omega)}(z, u)=\mathcal{T}_{\varphi}^{[N(\omega) u]}(z)=\sum_{j=1}^{N(\omega)} \varphi\left(\frac{j}{N(\omega)}\right) \mathcal{T}_{j}^{[N(\omega) u]}(z)
$$

where $\varphi \mapsto \mathcal{T}_{\varphi}^{N(\omega)}(z,$.$) can be extended into an operator from L^{2}(0,1)$ to itself.

TheOREM 6.5. We have

1. $\forall \varphi \in L^{2}(0,1)$ and $\forall z \geq 0$,

$$
\lim _{N(\omega) \rightarrow+\infty} \mathcal{T}_{\varphi}^{N(\omega)}(z, u)=\mathcal{T}_{\varphi}(z, u) \quad \text { in } L^{2}(0,1)
$$

where $\mathcal{T}_{\varphi}(z, u)$ satisfies the partial differential equation : $\forall(z, u) \in(0,+\infty) \times$ $(0,1)$,

$$
\frac{\partial}{\partial z} \mathcal{T}_{\varphi}(z, u)=\frac{\partial}{\partial u}\left(a_{\infty}(\cdot) \frac{\partial}{\partial u} \mathcal{T}_{\varphi}\right)(z, u)
$$

with the boundary conditions: $\forall z>0$

$$
\frac{\partial}{\partial u} \mathcal{T}_{\varphi}(z, 0)=0, \quad \frac{\partial}{\partial v} \mathcal{T}_{\varphi}(z, 1)=0, \quad \text { and } \quad \mathcal{T}_{\varphi}(0, u)=\varphi(u)
$$

2. $\forall u \in[0,1), \forall z \geq 0$, and $\forall \varphi \in \mathcal{C}^{0}([0,1])$ such that $\varphi(1)=0$, we have

$$
\lim _{N(\omega) \rightarrow+\infty} \mathcal{T}_{\varphi}^{N(\omega)}(z, u)=\mathcal{T}_{\varphi}(z, u) .
$$

Here,

$$
a_{\infty}(u)=\frac{a_{0}}{1-\left(1-\frac{\pi^{2}}{a^{2} d^{2}}\right)(\theta u)^{2}},
$$


with $a_{0}=\frac{\pi^{2} S_{0}}{2 a n_{1}^{4} d^{4} \theta^{2}}, \theta=\sqrt{1-1 / n_{1}^{2}}, S_{0}=\int_{0}^{d} \int_{0}^{d} \gamma_{0}\left(x_{1}, x_{2}\right) \cos \left(\frac{\pi}{d} x_{1}\right) \cos \left(\frac{\pi}{d} x_{2}\right) d x_{1} d x_{2} \cdot n_{1}$ is the index of refraction in the ocean section $[0, d], 1 / a$ is the correlation length of the random inhomogeneities in the longitudinal direction, and $\gamma_{0}$ is the covariance function of the random inhomogeneities in the transverse direction.

This theorem is a continuum approximation in the limit of a large number of propagating modes in the case where the radiation losses are negligible. This approximation gives us, in the limit of a large number of propagating modes $N(\omega) \gg 1$, a diffusion model for the transfer of energy between the $N(\omega)$-discrete propagating modes with two reflecting boundary conditions at $u=0$ (the top of the waveguide in Figure 2.1) and $u=1$ (the bottom of the waveguide in Figure 2.1). Here, the two reflecting boundary conditions mean that there is no radiative loss anymore (see Figure $6.2)$.

The proof of Theorem 6.5 uses the same method as the one of Theorem 6.2, and we refer to [12] for a complete proof of Theorem 6.5.

6.3.2. Asymptotic behavior of $\mathcal{T}(L, v)$ as $L \rightarrow+\infty$. In the case where the radiation losses are negligible, we have seen in Section 6.1 that the decay rate satisfies $\lim _{\tau \rightarrow 0} \Lambda_{\infty}^{\tau}(\omega)=0$ and $\mathcal{T}^{0, l}(\omega, L)$ converges to the uniform distribution over $\{1, \ldots, N(\omega)\}$ as $L \rightarrow+\infty[9]$. In the limit of a large number of propagating modes, we have the following continuous version.

Theorem 6.6. $\forall \varphi \in L^{2}(0,1)$ and $\forall u \in[0,1]$,

$$
\lim _{L \rightarrow+\infty} \mathcal{T}_{\varphi}(L, u)=\int_{0}^{1} \varphi(v) d v,
$$

that is, the energy carried by the continuum of propagating modes converges exponentially fast to the uniform distribution over $[0,1]$ as $L \rightarrow+\infty$. As a result, the energy is conserved and the modal energy distribution converges to a uniform distribution as $L \rightarrow+\infty$.

Proof. We can see that the operator $P_{\infty}=\frac{\partial}{\partial v}\left(a_{\infty}(\cdot) \frac{\partial}{\partial v}\right)$ on $L^{2}([0,1])$, with domain

$$
\mathcal{D}\left(P_{\infty}\right)=\left\{\varphi \in H^{2}(0,1), \quad \frac{\partial}{\partial v} \varphi(0)=0, \quad \frac{\partial}{\partial v} \varphi(1)=0\right\},
$$

is self-adjoint. Moreover, $P_{\infty}$ has a compact resolvant because $[0,1]$ is a compact set and then it has a point spectrum $\left(\lambda_{j}\right)_{j \geq 0}$ with eigenvectors denoted by $\left(\phi_{\infty, j}\right)_{j \geq 0}$. Moreover, all the eigenspaces are finite-dimensional subspaces of $\mathcal{D}\left(P_{\infty}\right)$ and $\forall \varphi \in$ $\mathcal{D}\left(P_{\infty}\right) \backslash\{0\}$,

$$
\left\langle P_{\infty}(\varphi), \varphi\right\rangle_{L^{2}(0,1)} \leq 0
$$

Let us remark that $\lambda_{0}=0$ is a simple eigenvalue with eigenvector $\phi_{\infty, 0}=1$. Then, the spectrum is included in $(-\infty, 0]$ and we have the following decomposition:

$$
\mathcal{T}_{\varphi}(z, v)=\int_{0}^{1} \varphi(v) d v+\sum_{j \geq 1}\left\langle\varphi, \phi_{\infty, j}\right\rangle_{L^{2}(0,1)} e^{\lambda_{j} z} \phi_{\infty, j}(v) .
$$

Therefore, $\forall u \in[0,1]$,

$$
\lim _{L \rightarrow+\infty} \mathcal{T}_{\varphi}(L, u)=\int_{0}^{1} \varphi(v) d v,
$$

with exponential rate $\lambda_{1}<0$. 
Conclusion. In this paper we have analyzed the propagation of waves in a shallow-water acoustic waveguide with random perturbations. In such a waveguide, the wave field can be decomposed into three kinds of modes, which are the propagating, the radiating, and the evanescent modes, and the random perturbations produce a coupling between these modes.

We have shown that the evolution of the propagating mode amplitudes can be described as a diffusion process (Theorems 5.4 and 5.5). This diffusion takes into account the main coupling mechanisms: the coupling with the evanescent modes induces a mode-dependent and frequency-dependent phase modulation on the propagating modes, and the coupling with the radiating modes, in addition to a modedependent and frequency-dependent phase modulation, induces a mode-dependent and frequency-dependent attenuation on the propagating modes. In other words, the propagating modes lose energy in the form of radiation into the bottom of the waveguide and their total energy decays exponentially with the propagation distance. We can express the decay rate in terms of a variational formula over a finite-dimensional space (Theorem 6.1).

Under the assumption that nearest neighbor-coupling is the main power transfer mechanism, the evolution of the mean mode powers of the propagating modes can be described, in the limit of a large number of propagating modes, by a continuous diffusive model with boundary conditions which take into account the effect of the radiation losses at the bottom and the free surface of the waveguide. In this regime, we observe that the energy carried by the continuum of propagating modes also decay exponentially with the propagation distance. The exponential decay rate can be expressed in terms of a variational formula (Theorem 6.3).

The diffusive systems obtained in this paper can be used to analyze pulse propagation and refocusing during time-reversal experiments in underwater acoustics [11].

\section{Appendix A.}

In this appendix, we present the sketches of the proofs of Theorems 5.4, 5.5 and 6.2. Full proofs of these theorems can be found in [12].

A.1. Proof of Theorem 5.4. The proof of this theorem is based on a martingale approach using the perturbed-test-function method. However, the process $\left(\mathbf{T}^{\xi, \epsilon}(z)\right)_{z \geq 0}$ is not adapted with respect to the filtration $\mathcal{F}_{z}^{\epsilon}=\mathcal{F}_{z / \epsilon}$. The proof of this theorem is in two parts. The first part of the proof consists in simplifying the problem and introducing a new process for which the martingale approach can be used. The first part of the proof follows the ideas of [16]. The second part of the proof of this theorem is based on a martingale approach using the perturbed-test-function method and follows the ideas developed in [4].

Let us introduce $\tilde{\mathbf{T}}^{\xi, \epsilon}($.$) - the unique solution of the differential equation$

$$
\frac{d}{d z} \tilde{\mathbf{T}}^{\xi, \epsilon}(z)=\frac{1}{\sqrt{\epsilon}} \mathbf{H}^{a a}\left(\frac{z}{\epsilon}\right) \tilde{\mathbf{T}}^{\xi, \epsilon}(\omega, z)+\left\langle\mathbf{G}^{a a}\right\rangle \tilde{\mathbf{T}}^{\xi, \epsilon}(z),
$$

with $\mathbf{T}^{\xi, \epsilon}(0)=I d$, and where $\left\langle\mathbf{G}^{a a}\right\rangle$ is defined, $\forall y \in \mathcal{H}_{\xi}$, by

$$
\left\langle\mathbf{G}^{a a}\right\rangle_{j}(y)=\int_{-\infty}^{-\xi} \frac{i k^{4}}{2 \beta_{j} \sqrt{\left|\gamma^{\prime}\right|}} \int_{0}^{+\infty} \mathbb{E}\left[C_{j \gamma^{\prime}}(0) C_{j \gamma^{\prime}}(z)\right] \cos \left(\beta_{j} z\right) e^{-\sqrt{\left|\gamma^{\prime}\right|}} d z d \gamma^{\prime} y_{j},
$$

$\forall j \in\{1, \ldots, N\}$, and $\left\langle\mathbf{G}^{a a}\right\rangle_{\gamma}(y)=0$ for almost every $\gamma \in\left(\xi, k^{2}\right)$. We have the following proposition that describes the relation between the two processes $\mathbf{T}^{\xi, \epsilon}(z)$ and $\tilde{\mathbf{T}}^{\xi, \epsilon}(z)$. 
Proposition A.1.

$$
\forall y \in \mathcal{H}_{\xi} \text { and } \forall \eta>0, \quad \lim _{\epsilon \rightarrow 0} \mathbb{P}\left(\sup _{z \in[0, L]}\left\|\mathbf{T}^{\xi, \epsilon}(z)(y)-\tilde{\mathbf{T}}^{\xi, \epsilon}(z)(y)\right\|_{\mathcal{H}_{\xi}^{\omega}}^{2}>\eta\right)=0 .
$$

Let us remark that the new process $\left(\tilde{\mathbf{T}}^{\xi, \epsilon}(z)\right)_{z \geq 0}$ is adapted to the filtration $\mathcal{F}_{z}^{\epsilon}$ and $\left\|\tilde{\mathbf{T}}^{\xi, \epsilon}(z)(y)\right\|_{\mathcal{H}_{\xi}}^{2}=\|y\|_{\mathcal{H}_{\xi}}^{2}, \forall z \geq 0$. Let $r_{y}=\|y\|_{\mathcal{H}_{\xi}}$,

$$
\mathcal{B}_{r_{y}, \mathcal{H}_{\xi}}=\left\{\lambda \in \mathcal{H}_{\xi},\|\lambda\|_{\mathcal{H}_{\xi}}=\sqrt{\langle\lambda, \lambda\rangle_{\mathcal{H}_{\xi}}} \leq r_{y}\right\},
$$

the closed ball with radius $r_{y}$, and $\left\{g_{n}, n \geq 1\right\}$ a dense subset of $\mathcal{B}_{r_{y}, \mathcal{H}_{\xi}}$. We equip $\mathcal{B}_{r_{y}, \mathcal{H}_{\xi}}$ with the distance $d_{\mathcal{B}_{r_{y}, \mathcal{H}_{\xi}}}$ defined by

$$
d_{\mathcal{B}_{r_{y}, \mathcal{H}_{\xi}}}(\lambda, \mu)=\sum_{j=1}^{+\infty} \frac{1}{2^{j}}\left|\left\langle\lambda-\mu, g_{n}\right\rangle_{\mathcal{H}_{\xi}}\right|
$$

$\forall(\lambda, \mu) \in \mathcal{B}_{r_{y}, \mathcal{H}_{\xi}}{ }^{2}$. As a result, $\left(\mathcal{B}_{\mathcal{H}_{\xi}}, d_{\mathcal{B}_{r_{y}}, \mathcal{H}_{\xi}}\right)$ is a compact metric space. From the definition of the metric $d_{\mathcal{B}_{r_{y}, \mathcal{H}_{\xi}}}$, we have the following criterion.

Theorem A.2. A family of processes $\left(X^{\epsilon}\right)_{\epsilon \in(0,1)}$ is tight in $\mathcal{C}\left([0,+\infty),\left(\mathcal{B}_{r_{y}, \mathcal{H}_{\xi}}, d_{\mathcal{B}_{r_{y}, \mathcal{H}_{\xi}}}\right)\right)$ if and only if $\left(\left\langle X^{\epsilon}, \lambda\right\rangle_{\mathcal{H}_{\xi}}\right)_{\epsilon \in(0,1)}$ is tight on $\mathcal{C}([0,+\infty), \mathbb{C})$ $\forall \lambda \in \mathcal{H}_{\xi}$.

This last theorem looks like the tightness criterion of Mitoma and Fouque [23, 6]. For any $\lambda \in \mathcal{H}_{\xi}$, we set $\tilde{\mathbf{T}}_{\lambda}^{\xi, \epsilon}(z)(y)=\left\langle\tilde{\mathbf{T}}^{\xi, \epsilon}(z)(y), \lambda\right\rangle_{\mathcal{H}_{\xi}}$. According to Theorem A.2, the family $\left(\tilde{\mathbf{T}}^{\xi, \epsilon}(.)(y)\right)_{\epsilon}$ is tight on $\mathcal{C}\left([0,+\infty),\left(\mathcal{B}_{r_{y}, \mathcal{H}_{\xi}}, d_{\mathcal{B}_{r_{y}}, \mathcal{H}_{\xi}}\right)\right)$ if and only if the family $\left(\tilde{\mathbf{T}}_{\lambda}^{\xi, \epsilon}(.)(y)\right)_{\epsilon}$ is tight on $\mathcal{C}([0,+\infty), \mathbb{C}) \forall \lambda \in \mathcal{H}_{\xi}$. Furthermore, $\left(\tilde{\mathbf{T}}^{\xi, \epsilon}(.)(y)\right)_{\epsilon}$ is a family of continuous processes, so it is sufficient to prove that, $\forall \lambda \in \mathcal{H}_{\xi},\left(\tilde{\mathbf{T}}_{\lambda}^{\xi, \epsilon}(.)(y)\right)_{\epsilon}$ is tight in the space of cad-lag functions $\mathcal{D}([0,+\infty), \mathbb{C})$ equipped with the Skorokhod topology. According to Proposition A.1, to study the convergence in distribution of the process $\left(\mathbf{T}^{\xi, \epsilon}(.)(y)\right)_{\epsilon}$ it suffices to study this convergence for $\left(\tilde{\mathbf{T}}^{\xi, \epsilon}(.)(y)\right)_{\epsilon}$.

We consider the complex case for more convenient manipulations. The proof of this theorem is based on the perturbed-test-function approach. Using the notion of a pseudogenerator, we prove tightness and characterize all subsequence limits.

We recall the techniques developed by Kurtz and Kushner. Let $\mathcal{M}^{\epsilon}$ be the set of all $\mathcal{F}^{\epsilon}$-measurable functions $f(t)$ for which $\sup _{t \leq T} \mathbb{E}[|f(t)|]<+\infty$ and where $T>0$ is fixed. The $p-\lim$ and the pseudogenerator are defined as follows. Let $f$ and $f^{\delta}$ be in $\mathcal{M}^{\epsilon} \forall \delta>0$. We say that $f=p-\lim _{\delta} f^{\delta}$ if

$$
\sup _{t, \delta} \mathbb{E}\left[\left|f^{\delta}(t)\right|\right]<+\infty \quad \text { and } \quad \lim _{\delta \rightarrow 0} \mathbb{E}\left[\left|f^{\delta}(t)-f(t)\right|\right]=0 \quad \forall t .
$$

The domain of $\mathcal{A}^{\epsilon}$ is denoted by $\mathcal{D}\left(\mathcal{A}^{\epsilon}\right)$. We say that $f \in \mathcal{D}\left(\mathcal{A}^{\epsilon}\right)$ and $\mathcal{A}^{\epsilon} f=g$ if $f$ and $g$ are in $\mathcal{D}\left(\mathcal{A}^{\epsilon}\right)$ and

$$
p-\lim _{\delta \rightarrow 0}\left[\frac{\mathbb{E}_{t}^{\epsilon}[f(t+\delta)]-f(t)}{\delta}-g(t)\right]=0
$$


where $\mathbb{E}_{t}^{\epsilon}$ is the conditional expectation given $\mathcal{F}_{t}^{\epsilon}$ and $\mathcal{F}_{t}^{\epsilon}=\mathcal{F}_{t / \epsilon}$. A useful result about $\mathcal{A}^{\epsilon}$ is given by the following theorem.

Theorem A.3. Let $f \in \mathcal{D}\left(\mathcal{A}^{\epsilon}\right)$. Then

$$
M_{f}^{\epsilon}(t)=f(t)-\int_{0}^{t} \mathcal{A}^{\epsilon} f(u) d u
$$

is an $\left(\mathcal{F}_{t}^{\epsilon}\right)$-martingale.

Proposition A.4. $\forall \lambda \in \mathcal{H}_{\xi}$, the family $\left(\tilde{\mathbf{T}}_{\lambda}^{\xi, \epsilon}(.)(y)\right)_{\epsilon \in(0,1)}$ is tight in $\mathcal{D}([0,+\infty), \mathbb{C})$.

Proof. First, we easily obtain $\forall T>0$,

$$
\lim _{M \rightarrow+\infty} \varlimsup_{\epsilon \rightarrow 0} \mathbb{P}\left(\sup _{0 \leq t \leq T}\left|\tilde{\mathbf{T}}_{\lambda}^{\xi, \epsilon}(t)(y)\right| \geq M\right)=0
$$

since $\left(\tilde{\mathbf{T}}^{\xi, \epsilon}(.)(y)\right)_{\epsilon}$ is a bounded process. Second, in [12] we build a function $f_{1}^{\epsilon}(t)$ such that $\forall T>0, \lim _{\epsilon} \sup _{0 \leq t \leq T}\left|f_{1}^{\epsilon}(t)\right|=0$ in probability and $\left\{\mathcal{A}^{\epsilon}\left(f_{0}^{\epsilon}+f_{1}^{\epsilon}\right)(t)\right\}_{\epsilon, 0 \leq t \leq T}$ is uniformly integrable. According to Theorem 4 [18], these two facts imply the tightness of $\left(\tilde{\boldsymbol{T}}_{\lambda}^{\xi, \epsilon}(.)(y)\right)_{\epsilon \in(0,1)}$.

We shall consider the classical complex derivative with the following notation: If $v=\alpha+i \beta$, then $\partial_{v}=\frac{1}{2}\left(\partial_{\alpha}-i \partial_{\beta}\right)$ and $\partial_{\bar{v}}=\frac{1}{2}\left(\partial_{\alpha}+i \partial_{\beta}\right)$. Let $f$ be a smooth function and $f_{0}^{\epsilon}(t)=f\left(\tilde{\mathbf{T}}_{\lambda}^{\xi, \epsilon}(t)(y)\right)$.

Now, we shall characterize all subsequence limits by showing they are solutions of a well-posed martingale problem. To do that, we consider a convergent subsequence of $\left(\tilde{\mathbf{T}}^{\xi, \epsilon}(.)(y)\right)_{\epsilon \in(0,1)}$ which converges to a limit $\mathbf{T}^{\xi}().(y)$. For the sake of simplicity we denote the subsequence by $\left(\tilde{\mathbf{T}}^{\xi, \epsilon}(.)(y)\right)_{\epsilon \in(0,1)}$.

Proposition A.5. $\forall \lambda \in \mathcal{H}_{\xi}$ and for all smooth test functions $f$,

$$
\begin{aligned}
f\left(\mathbf{T}_{\lambda}^{\xi}(t)(y)\right) & -\int_{0}^{t} \partial_{v} f\left(\mathbf{T}_{\lambda}^{\xi}(s)(y)\right)\left\langle J^{\xi}\left(\mathbf{T}^{\xi}(s)(y)\right), \lambda\right\rangle_{\mathcal{H}_{\xi}} \\
& +\partial_{\bar{v}} f\left(\mathbf{T}_{\lambda}^{\xi}(s)(y)\right) \overline{\left\langle J^{\xi}\left(\mathbf{T}^{\xi}(s)(y)\right), \lambda\right\rangle_{\mathcal{H}_{\xi}}} \\
& +\partial_{v}^{2} f\left(\mathbf{T}_{\lambda}^{\xi}(s)(y)\right)\left\langle K\left(\mathbf{T}^{\xi}(s)(y)\right)(\lambda), \lambda\right\rangle_{\mathcal{H}_{\xi}} \\
& +\partial_{\bar{v}}^{2} f\left(\mathbf{T}_{\lambda}^{\xi}(s)(y)\right) \overline{\left\langle K\left(\mathbf{T}^{\xi}(s)(y)\right)(\lambda), \lambda\right\rangle_{\mathcal{H}_{\xi}}} \\
& +\partial_{\bar{v}} \partial_{v} f\left(\mathbf{T}_{\lambda}^{\xi}(s)(y)\right)\left\langle L\left(\mathbf{T}^{\xi}(s)(y)\right)(\lambda), \lambda\right\rangle_{\mathcal{H}_{\xi}} \\
& +\partial_{v} \partial_{\bar{v}} f\left(\mathbf{T}_{\lambda}^{\xi}(s)(y)\right) \overline{\left\langle L\left(\mathbf{T}^{\xi}(s)(y)\right)(\lambda), \lambda\right\rangle_{\mathcal{H}_{\xi}}} d s
\end{aligned}
$$


is a martingale, where

$$
\begin{aligned}
J^{\xi}(\mathbf{T})_{j} & =\left[\frac{\Gamma_{j j}^{c}-\Gamma_{j j}^{1}}{2}-\Lambda_{j}^{c, \xi}+i\left(\frac{\Gamma_{j j}^{s}}{2}-\Lambda_{j}^{s, \xi}+\kappa_{j}^{\xi}\right)\right] \mathbf{T}_{j}, \\
K(\mathbf{T})(\lambda)_{j} & =-\frac{1}{2} \sum_{l=1}^{N} \Gamma_{j l}^{1} \mathbf{T}_{j} \mathbf{T}_{l} \overline{\lambda_{l}}-\frac{1}{2} \sum_{\substack{l=1 \\
l \neq j}}^{N}\left(\Gamma_{j l}^{c}+i \Gamma_{j l}^{s}\right) \mathbf{T}_{j} \mathbf{T}_{l} \overline{\lambda_{l}}, \\
L(\mathbf{T})(\lambda)_{j} & =\frac{1}{2} \sum_{l=1}^{N} \Gamma_{j l}^{1} \mathbf{T}_{j} \overline{\mathbf{T}_{l}} \lambda_{l}+\frac{1}{2} \sum_{\substack{l=1 \\
l \neq j}}^{N} \Gamma_{j l}^{c} \mathbf{T}_{l} \overline{\mathbf{T}_{l}} \lambda_{j},
\end{aligned}
$$

and $J^{\xi}(\mathbf{T})_{\gamma}=K(\mathbf{T})(\lambda)_{\gamma}=L(\mathbf{T})(\lambda)_{\gamma}=0$ for almost every $\gamma \in\left(\xi, k^{2}\right)$, and for $(\mathbf{T}, \lambda) \in$ $\mathcal{H}_{\xi}^{2}$.

Proof. Let, for $\mu>0$ and $j \in\{1,2,3,4\}$,

$$
\begin{aligned}
& I_{<\mu}^{j}=\left\{\left(\gamma_{l}\right)_{l \in\{1, \ldots, j\}} \in\left(\xi, k^{2}\right)^{j}, \exists\left(q_{l}\right)_{l \in\{1, \ldots, 4-j\}} \in\left\{\beta_{1}, \ldots, \beta_{N}\right\}^{4-j}\right. \\
& \left.\quad \text { and }\left(\mu_{l}\right)_{l \in\{1, \ldots, 4\}} \in\{-1,1\}^{4}, \text { with }\left|\sum_{l=1}^{j} \mu_{l} \sqrt{\gamma_{l}}+\sum_{l=1}^{4-j} \mu_{l+j} q_{l}\right|<\mu\right\} .
\end{aligned}
$$

In addition to $f_{1}^{\epsilon}(t)$, which also satisfies $\sup _{t \geq 0} \mathbb{E}\left[\left|f_{1}^{\epsilon}(t)\right|\right]=\mathcal{O}(\sqrt{\epsilon})$, there exists $f_{2}^{\epsilon}(t)$ and $f_{3}^{\epsilon}(t)$, such that $\sup _{t \geq 0} \mathbb{E}\left[\left|f_{2}^{\epsilon}(t)\right|\right]=\mathcal{O}(\epsilon)$ and

$$
\varlimsup_{\epsilon \rightarrow 0} \sup _{0 \leq t \leq T} \mathbb{E}\left[\left|f_{3}^{\epsilon}(t)\right|\right] \leq K(T, \xi, y) \sum_{j=1}^{4} \int_{I_{<\mu}^{j}} d \gamma_{1} \ldots d \gamma_{j},
$$

$\forall T>0$, and

$$
\begin{aligned}
\mathcal{A}^{\epsilon} f^{\epsilon}(t)= & \partial_{v} f\left(\tilde{\mathbf{T}}_{\lambda}^{\xi, \epsilon}(t)(y)\right)\left\langle J^{\xi}\left(\tilde{\mathbf{T}}^{\xi, \epsilon}(t)(y)\right), \lambda\right\rangle_{\mathcal{H}} \\
& +\partial_{\bar{v}} f\left(\tilde{\mathbf{T}}_{\lambda}^{\xi, \epsilon}(t)(y)\right) \overline{\left\langle J^{\xi}\left(\tilde{\mathbf{T}}^{\xi, \epsilon}(t)(y)\right), \lambda\right\rangle_{\mathcal{H}}} \\
& +\partial_{v}^{2} f\left(\tilde{\mathbf{T}}_{\lambda}^{\xi, \epsilon}(t)(y)\right)\left\langle K\left(\tilde{\mathbf{T}}^{\xi, \epsilon}(t)(y)\right)(\lambda), \lambda\right\rangle_{\mathcal{H}} \\
& +\partial_{\bar{v}}^{2} f\left(\tilde{\mathbf{T}}_{\lambda}^{\xi, \epsilon}(t)(y)\right) \overline{\left\langle K\left(\tilde{\mathbf{T}}^{\xi, \epsilon}(t)(y)\right)(\lambda), \lambda\right\rangle_{\mathcal{H}}} \\
& +\partial_{\bar{v}} \partial_{v} f\left(\tilde{\mathbf{T}}_{\lambda}^{\xi, \epsilon}(t)(y)\right)\left\langle L\left(\tilde{\mathbf{T}}^{\xi, \epsilon}(t)(y)\right)(\lambda), \lambda\right\rangle_{\mathcal{H}} \\
& +\partial_{v} \partial_{\bar{v}} f\left(\tilde{\mathbf{T}}_{\lambda}^{\xi, \epsilon}(t)(y)\right) \frac{\left\langle L\left(\tilde{\mathbf{T}}^{\xi, \epsilon}(t)(y)\right)(\lambda), \lambda\right\rangle_{\mathcal{H}}}{\langle} \\
& +C(\epsilon, t),
\end{aligned}
$$

for $f^{\epsilon}(t)=f_{0}^{\epsilon}(t)+f_{1}^{\epsilon}(t)+f_{2}^{\epsilon}(t)+f_{3}^{\epsilon}(t)$. Moreover

$$
\varlimsup_{\epsilon \rightarrow 0} \sup _{0 \leq t \leq T} \mathbb{E}[|C(\epsilon, t)|] \leq K(T, \xi, y) \sum_{j=1}^{4} \int_{I_{<\mu}^{j}} d \gamma_{1} \ldots d \gamma_{j} .
$$

To get this expression we have supposed that the following nondegeneracy condition holds: the wavenumbers $\beta_{j}$ are distinct along with their sums and differences. 
Therefore, by Theorem A.3, $\left(M_{f^{\epsilon}}^{\epsilon}(t)\right)_{t>0}$ is an $\left(\mathcal{F}_{t}^{\epsilon}\right)$-martingale. Then for every bounded continuous function $h$, every sequence $0<s_{1}<\cdots<s_{n} \leq s<t$, and every family $\left(\lambda_{j}\right)_{j \in\{1, \ldots, n\}}$ with values in $\mathcal{H}_{\xi}^{n}$ we have

$$
\mathbb{E}\left[h\left(\tilde{\mathbf{T}}_{\lambda_{j}}^{\xi, \epsilon}\left(s_{j}\right)(y), 1 \leq j \leq n\right)\left(f^{\epsilon}(t)-f^{\epsilon}(s)-\int_{s}^{t} \mathcal{A}^{\epsilon} f^{\epsilon}(u) d u\right)\right]=0 .
$$

Finally, by letting $\epsilon \rightarrow 0$ and $\mu \rightarrow 0$, we get the desired result.

In order to prove uniqueness, we decompose $\mathbf{T}^{\xi}().(y)$ into real and imaginary parts. Let us consider the new process

$$
\mathbf{Y}^{\xi}(t)=\left[\begin{array}{l}
\mathbf{Y}^{1, \xi}(t) \\
\mathbf{Y}^{2, \xi}(t)
\end{array}\right], \text { where } \mathbf{Y}^{1, \xi}(t)=\operatorname{Re}\left(\mathbf{T}^{\xi}(t)(y)\right) \text { and } \mathbf{Y}^{2, \xi}(t)=\operatorname{Im}\left(\mathbf{T}^{\xi}(t)(y)\right)
$$

This new process takes its values in $\mathcal{G}_{\xi} \times \mathcal{G}_{\xi}$, where $\mathcal{G}_{\xi}=\mathbb{R}^{N} \times L^{2}\left(\left(\xi, k^{2}\right), \mathbb{R}\right)$, and we introduce the operator

$$
\begin{array}{r}
\Upsilon: \mathcal{G}_{\xi} \times \mathcal{G}_{\xi} \longrightarrow \mathcal{G}_{\xi} \times \mathcal{G}_{\xi}, \\
{\left[\begin{array}{c}
\mathbf{T}^{1} \\
\mathbf{T}^{2}
\end{array}\right] \longmapsto\left[\begin{array}{c}
\mathbf{T}^{2} \\
-\mathbf{T}^{1}
\end{array}\right] .}
\end{array}
$$

Proposition A.6. $\forall f \in \mathcal{C}_{b}^{2}\left(\mathcal{G}_{\xi} \times \mathcal{G}_{\xi}\right)$,

$$
M_{f}^{\xi}(t)=f\left(\mathbf{Y}^{\xi}(t)\right)-\int_{0}^{t} L^{\xi} f\left(\mathbf{Y}^{\xi}(s)\right) d s
$$

is a continuous martingale, where $\forall(\mathbf{Y}, \lambda) \in\left(\mathcal{G}_{\xi} \times \mathcal{G}_{\xi}\right)^{2}$,

$$
L^{\xi} f(\mathbf{Y})=\frac{1}{2} \operatorname{trace}\left(A(\mathbf{Y}) D^{2} f(\mathbf{Y})\right)+\left\langle J^{\xi}(\mathbf{Y}), D f(\mathbf{Y})\right\rangle_{\mathcal{G}_{\xi} \times \mathcal{G}_{\xi}}
$$

with $A(\mathbf{Y})(\lambda)=A_{1}(\mathbf{Y})(\lambda)+A_{2}(\mathbf{Y})(\lambda)+A_{3}(\mathbf{Y})(\lambda)$. Moreover, for $j \in\{1, \ldots, N\}$,

$$
\begin{aligned}
B^{\xi}(\mathbf{Y})_{j} & =\left[\frac{\Gamma_{j j}^{c}}{2}-\Lambda_{j}^{c, \xi}\right] \mathbf{Y}_{j}-\left[\frac{\Gamma_{j j}^{s}}{2}+\kappa_{j}^{\xi}-\Lambda_{j}^{s, \xi}\right] \Upsilon_{j}(\mathbf{Y}), \\
A_{1}(\mathbf{Y})(\lambda)_{j} & =\Upsilon_{j}(\mathbf{Y}) \sum_{l=1}^{N} \Gamma_{j l}^{1}\left[\Upsilon_{l}^{1}(\mathbf{Y}) \lambda_{l}^{1}+\Upsilon_{l}^{2}(\mathbf{Y}) \lambda_{l}^{2}\right] \\
A_{2}(\mathbf{Y})(\lambda)_{j} & =-\mathbf{Y}_{j} \sum_{\substack{l=1 \\
l \neq j}}^{N} \Gamma_{j l}^{c}\left[\mathbf{Y}_{l}^{1} \lambda_{l}^{1}+\mathbf{Y}_{l}^{2} \lambda_{l}^{2}\right]+\Upsilon_{j}(\mathbf{Y}) \sum_{\substack{l=1 \\
l \neq j}}^{N} \Gamma_{j l}^{c}\left[\Upsilon_{l}^{1}(\mathbf{Y}) \lambda_{l}^{1}+\Upsilon_{l}^{2}(\mathbf{Y}) \lambda_{l}^{2}\right] \\
A_{3}(\mathbf{Y})(\lambda)_{j} & =\lambda_{j} \sum_{\substack{l=1 \\
l \neq j}}^{N} \Gamma_{j l}^{c}\left[\left(\mathbf{Y}_{l}^{1}\right)^{2}+\left(\mathbf{Y}_{l}^{2}\right)^{2}\right]
\end{aligned}
$$

and $B_{\gamma}^{\xi}(\mathbf{Y})=A_{\gamma}(\mathbf{Y})(\lambda)=A_{\gamma}(\mathbf{Y})(\lambda)=A_{\gamma}(\mathbf{Y})(\lambda)=0$ for almost every $\gamma \in\left(\xi, k^{2}\right)$. Moreover, the martingale problem associated to the generator $L^{\xi}$ is well-posed.

Proof. Following the proof of Theorem 4.1.4 in [32], to prove that $M_{f}^{\xi}$ is a martingale it suffices to show that

$$
\left\langle M^{\xi}(t), \lambda\right\rangle_{\mathcal{G}_{\xi} \times \mathcal{G}_{\xi}}=M_{\lambda}^{\xi}(t)=\left\langle\mathbf{Y}^{\xi}(t)-\int_{0}^{t} B^{\xi}\left(\mathbf{Y}^{\xi}(s)\right) d s, \lambda\right\rangle_{\mathcal{G}_{\xi} \times \mathcal{G}_{\xi}}
$$


is a continuous martingale with quadratic variation

$$
<M_{\lambda}^{\xi}>(t)=\int_{0}^{t}\left\langle A\left(\mathbf{Y}^{\xi}(s)\right)(\lambda), \lambda\right\rangle_{\mathcal{G}_{\xi} \times \mathcal{G}_{\xi}} d s
$$

Moreover, $\forall(\mathbf{Y}, \lambda) \in\left(\mathcal{G}_{\xi} \times \mathcal{G}_{\xi}\right)^{2}$, we have $\langle A(\mathbf{Y})(\lambda), \lambda\rangle_{\mathcal{G}_{\xi} \times \mathcal{G}_{\xi}} \geq 0$ and $\operatorname{trace}(A(\mathbf{Y}))<$ $+\infty$. According to Theorem 3.2.2 and 4.4.1 in [32], the martingale problem associated to $L^{\xi}$ is well-posed.

A.2. Proof of Theorem 5.5. Let $\mathcal{H}_{0}=\mathbb{C}^{N} \times L^{2}\left(0, k^{2}\right), y \in \mathcal{H}_{0}$, and $y^{\xi}=$ $\Pi(\xi,+\infty)(y)$. As the radiating part $\Pi\left(0, k^{2}\right)\left(\mathbf{T}^{\xi}().\left(y^{\xi}\right)\right)$ of the process $\mathbf{T}^{\xi}().\left(y^{\xi}\right)$ is constant equal to $\Pi\left(\xi, k^{2}\right)\left(y^{\xi}\right)$, to prove the tightness of $\left(\mathbf{T}^{\xi}(.)\left(y^{\xi}\right)\right)_{\xi}$ is suffices to show the tightness of the finite-dimensional process $\left(\Pi\left(k^{2},+\infty\right)\left(\mathbf{T}^{\xi}(.)\left(y^{\xi}\right)\right)\right)_{\xi}$. The tightness is ensured by the fact that the process $\mathbf{T}^{\xi}().\left(y^{\xi}\right)$ takes its values in $\mathcal{B}_{r_{y}, \mathcal{H}_{\xi}}$ and is the solution of the martingale problem associated to $L^{\xi}$ and starting from $y^{\xi}$.

Now, to characterize all limits of converging subsequences, let us denote by $\mathbf{T}^{0}().(y)$ such a limit point. First, for every smooth function $f$ on $\mathcal{H}_{0}$, for every bounded continuous function $h$, and every sequence $0<s_{1}<\cdots<s_{n} \leq s<t$, we have

$$
\begin{aligned}
\mathbb{E} & {\left[h\left(\mathbf{T}^{\xi}\left(s_{j}\right)\left(y^{\xi}\right), 1 \leq j \leq n\right)\right.} \\
& \left.\times\left(f\left(\mathbf{T}^{\xi}(t)\left(y^{\xi}\right)\right)-f\left(\mathbf{T}^{\xi}(s)\left(y^{\xi}\right)\right)-\int_{s}^{t} \mathcal{L}_{\xi} f\left(\mathbf{T}^{\xi}(u)\left(y^{\xi}\right)\right) d u\right)\right]=0 .
\end{aligned}
$$

Second,

$$
\sup _{\mathbf{T} \in \mathcal{B}_{r_{y}, \mathcal{H}_{0}}}\left|\mathcal{L} f(\mathbf{T})-\mathcal{L}_{\xi} f(\mathbf{T})\right| \leq K \sup _{j \in\{1, \ldots, N\}}\left|\Lambda_{j}^{c, \xi}-\Lambda_{j}^{c}\right|+\left|\Lambda_{j}^{s, \xi}-\Lambda_{j}^{s}\right|+\left|\kappa_{j}^{\xi}-\kappa_{j}\right| .
$$

Consequently, $\mathbf{T}^{0}().(y)$ is a solution of the martingale problem associated to $\mathcal{L}$ and starting from $y$. With the same arguments as in the proof of the uniqueness in Theorem 5.4, this martingale problem is well-posed and therefore $\mathbf{T}^{\xi}().\left(y^{\xi}\right)$ converges in distribution to the unique solution of the martingale problem associated to $\mathcal{L}$ and starting from $y$.

A.3. Proof of Theorem 6.2. The proof of this theorem follows the ideas developed in [29, Chapter 11]. In order to prove this theorem we use a probabilistic representation of $\mathcal{T}_{j}^{l}(\omega, z)$ by using the Feynman-Kac formula. To this end, we introduce the jump Markov process $\left(X_{t}^{N}\right)_{t \geq 0}$ with state space $\{-(N-1) / N, \ldots, 0, \ldots,(N-1) / N\}$ and generator given by

$$
\mathcal{L}^{N} \phi\left(\frac{l}{N}\right)=\Gamma_{l, l+1}^{c}\left(\phi\left(\frac{l-1}{N}\right)-\phi\left(\frac{l}{N}\right)\right)+\Gamma_{l+2, l+1}^{c}\left(\phi\left(\frac{l+1}{N}\right)-\phi\left(\frac{l}{N}\right)\right)
$$

for $l \in\{1, \ldots, N-2\}$,

$$
\mathcal{L}^{N} \phi\left(\frac{l}{N}\right)=\Gamma_{|l|+2,|l|+1}^{c}\left(\phi\left(\frac{l-1}{N}\right)-\phi\left(\frac{l}{N}\right)\right)+\Gamma_{|l|,|l|+1}^{c}\left(\phi\left(\frac{l+1}{N}\right)-\phi\left(\frac{l}{N}\right)\right)
$$

for $l \in\{-(N-2), \ldots,-1\}$,

$$
\mathcal{L}^{N} \phi(0)=\frac{\Gamma_{2,1}^{c}}{2}\left(\phi\left(\frac{1}{N}\right)-\phi(0)\right)+\frac{\Gamma_{2,1}^{c}}{2}\left(\phi\left(\frac{-1}{N}\right)-\phi(0)\right),
$$


and

$$
\mathcal{L}^{N} \phi\left(\frac{ \pm(N-1)}{N}\right)=\Gamma_{N-1, N}^{c}\left(\phi\left(\frac{ \pm(N-2)}{N}\right)-\phi\left(\frac{ \pm(N-1)}{N}\right)\right) .
$$

Using the Feynman-Kac formula we obtain, for $(j, l) \in\{1, \ldots, N\}^{2}$,

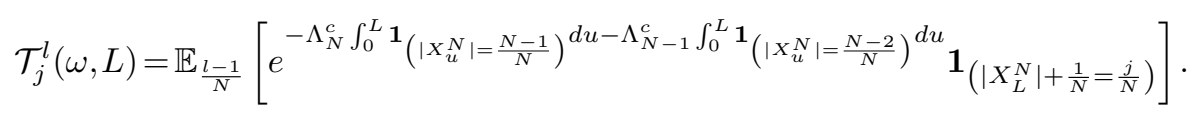

Let $f$ be a bounded continuous function on $[0,1]$, and consider $\mathcal{T}^{l}(\omega, L)$ as a family of bounded measures on $[0,1]$ by setting

$$
\mathcal{T}_{f}^{l}(\omega, L)=\mathbb{E}_{\frac{l-1}{N}}\left[e^{-\Lambda_{N}^{c} \int_{0}^{L} \mathbf{1}\left(\left|X_{u}^{N}\right|=\frac{N-1}{N}\right) d u-\Lambda_{N-1}^{c} \int_{0}^{L} \mathbf{1}\left(\left|X_{u}^{N}\right|=\frac{N-2}{N}\right) d u} f\left(\left|X_{L}^{N}\right|+\frac{1}{N}\right)\right] .
$$

In the first part of the proof we consider the case $u \in[0,1)$, and in the second part we shall treat the case $u=1$.

Let $u \in[0,1)$ such that $l(N) / N \rightarrow u$. We begin by introducing some notations. Throughout the proof we denote by $\tau_{j / N}^{(l)}$ the $l$ th passage in $j / N$, for $j \in\{-(N-$ $1), \ldots, N-1\}$. To avoid the unboundness in $\mathcal{L}^{N}$ of the reflecting barriers $\mathcal{L}^{N} \phi( \pm(N-$ $1) / N)$, we introduce the stopping time

$$
\tau_{N}^{\alpha}=\tau_{\left(N-\left[N^{\alpha}\right]\right) / N}^{(1)} \wedge \tau_{-\left(N-\left[N^{\alpha}\right]\right) / N}^{(1)}
$$

with $\alpha \in(0,1)$. Let $X_{t}^{N, \tau}=X_{t \wedge \tau_{N}^{\alpha}}^{N}, \forall t \geq 0$, be the stopped process and $d(N)=(l(N)-$ $1) / N$. We denote by $\mathbb{P}_{d(N)}^{N}$ the law of $\left(X_{t}^{N}\right)_{t \geq 0}$ starting from $d(N)$ and by $\mathbb{P}_{d(N)}^{N, \tau}$ the law of $\left(X_{t}^{N, \tau}\right)_{t \geq 0}$ starting from $d(N)$. Let

$$
\mathcal{L}_{\bar{a}_{\infty}}=\frac{\partial}{\partial v}\left(\bar{a}_{\infty}(\cdot) \frac{\partial}{\partial v}\right)
$$

where $\bar{a}_{\infty}(\cdot) \in \mathcal{C}^{1}(\mathbb{R})$ is an extension over $\mathbb{R}$ of $a_{\infty}(\cdot)$, which is defined on $[-1,1]$, and such that the martingale problem associated to $\mathcal{L}_{\bar{a}_{\infty}}$ and starting from $v$ is well posed. We denote by $\overline{\mathbb{P}}_{v}$ this unique solution. Using Lemma 3.1 we have the following result about the convergence of the generator $\mathcal{L}^{N}$.

Lemma A.7. $\forall \varphi \in \mathcal{C}_{0}^{\infty}(\mathbb{R}), \forall \alpha \in(2 / 3,1)$,

$$
\lim _{N \rightarrow+\infty} \sup _{v \in\left[-\frac{N-\left[N^{\alpha}\right]}{N},-\frac{1}{N}\right] \cup\left[\frac{1}{N}, \frac{N-\left[N^{\alpha}\right]}{N}\right]}\left|\mathcal{L}^{N} \varphi(v)-\mathcal{L}_{a_{\infty}} \varphi(v)\right|=0,
$$

where $\mathcal{L}^{N} \varphi(v)$ is defined as follows. $\forall j \in\{1, \ldots, N-2\}$,

$$
\begin{aligned}
\mathcal{L}^{N} \varphi(v)= & \Gamma_{j, j+1}^{c}\left(\varphi\left(\frac{j-1}{N}\right)-\varphi\left(\frac{j}{N}\right)\right) \\
& +\Gamma_{j+1, j+2}^{c}\left(\varphi\left(\frac{j+1}{N}\right)-\varphi\left(\frac{j}{N}\right)\right)
\end{aligned}
$$

for $v \in[j / N,(j+1) / N)$, and

$$
\begin{aligned}
\mathcal{L}^{N} \varphi(v)= & \Gamma_{j, j+1}^{c}\left(\varphi\left(\frac{-j+1}{N}\right)-\varphi\left(\frac{-j}{N}\right)\right) \\
& +\Gamma_{j+1, j+2}^{c}\left(\varphi\left(\frac{-j-1}{N}\right)-\varphi\left(\frac{-j}{N}\right)\right)
\end{aligned}
$$


for $v \in(-(j+1) / N,-j / N]$.

A full proof of Lemma A.7 is given in [12]. As a result, we get the following lemma.

Lemma A.8. $\mathbb{P}_{d(N)}^{N, \tau}$ is tight on $\mathcal{D}([0,+\infty), \mathbb{R})$.

Proof. According to Theorem 3 in [18, Chapter 3], this lemma is a consequence of the two following facts. First, the process $\left(X_{t}^{N, \tau}\right)$ is bounded by $\left(N-\left[N^{\alpha}\right]\right) / N$. Second, we use the previous lemma and the fact $\left(X_{t}^{N}\right)$ satisfies a martingale problem associated to $\mathcal{L}^{N}$.

Let $\mathcal{M}_{t}=\sigma(x(u), 0 \leq u \leq t)$ and $\tau_{r}=\inf (u \geq 0,|x(t)| \geq r)$ for $r \in(0,1)$. Moreover, for $\varphi \in \mathcal{C}_{0}^{\infty}(\mathbb{R})$ we set

$$
M_{\varphi}(t)=\varphi(x(t))-\varphi(0)-\int_{0}^{t} \mathcal{L}_{\bar{a}_{\infty}} \varphi(x(u)) d u .
$$

Lemma A.9. Let $\mathbb{Q}_{u}$ be a limit point of the relatively compact sequence $\left(\mathbb{P}_{d(N)}^{N, \tau}\right)_{N}$. Then, $\forall \varphi \in \mathcal{C}_{0}^{\infty}(\mathbb{R})$ and $\forall r \in(0,1),\left(M_{\varphi}\left(t \wedge \tau_{r}\right)\right)_{t \geq 0}$ is a $(\mathcal{M})_{t}$-martingale under $\mathbb{Q}_{u}$.

Proof. Let $\left(\mathbb{P}_{d\left(N^{\prime}\right)}^{N^{\prime}, \tau}\right)_{N^{\prime}}$ be a converging subsequence. Let $0 \leq t_{1}<t_{2}$ and $\Phi$ be a bounded continuous $\mathcal{M}_{t_{1}}$-measurable function. We have

$$
\mathbb{E}^{\mathbb{P}_{d\left(N^{\prime}\right)}^{N^{\prime}, \tau}}\left(M_{\varphi}^{N^{\prime}}\left(t_{2} \wedge \tau_{r}\right) \Phi\right)=\mathbb{E}^{\mathbb{P}_{d\left(N^{\prime}\right)}^{N^{\prime}, \tau}}\left(M_{\varphi}^{N^{\prime}}\left(t_{1} \wedge \tau_{r}\right) \Phi\right)
$$

Lemma A.7 gives the convergence of the generator $\mathcal{L}^{N^{\prime}}$. However, to correct the problem in 0 we have, $\forall \alpha^{\prime} \in(0,1)$,

$$
\mathbb{E}_{0}\left[\int_{0}^{t} \mathbf{1}_{\left(X_{u}^{N^{\prime}}=0\right)} d u\right]=\mathcal{O}\left(\frac{1}{N^{\prime \alpha^{\prime} \wedge\left(1-\alpha^{\prime}\right)}}\right) .
$$

To finish the proof, we prove a version of Lemma 11.1.1 in [29] adapted to the Skorokhod topology on $\mathcal{D}([0,+\infty), \mathbb{R})[12]$.

The full details of the proof of Lemmas A.8 and A.9 can be found in [12]. From Lemma A.9, we have $\forall r \in(0,1), \mathbb{Q}_{u}=\overline{\mathbb{P}}_{u}$ on $\mathcal{M}_{\tau_{r}}$. Then, $\mathbb{Q}_{u}=\overline{\mathbb{P}}_{u}$ on $\mathcal{M}_{\tau_{1}}$ since $\tau_{r} \nearrow \tau_{1}$ as $r \nearrow 1$, and $\mathbb{Q}_{u}(\mathcal{C}([0,+\infty), \mathbb{R}))=\overline{\mathbb{P}}_{u}(\mathcal{C}([0,+\infty), \mathbb{R}))=1$.

Let $f \in \mathcal{C}^{0}([0,1])$ with compact support included in $[0,1)$, and let $\left(\mathbb{P}_{d\left(N^{\prime}\right)}^{N^{\prime}, \tau}\right)_{N^{\prime}}$ be a converging subsequence as in the previous proof. We have

$$
\mathcal{T}_{f}^{l\left(N^{\prime}\right)}(\omega, t)=\mathbb{E}_{d\left(N^{\prime}\right)}\left[f\left(\left|X_{t}^{N^{\prime}}\right|+\frac{1}{N^{\prime}}\right) \mathbf{1}_{\left(t<\tau_{N^{\prime}}^{\alpha}\right)}\right]+r\left(N^{\prime}\right)
$$

with

$$
\begin{aligned}
r(N)=\mathbb{E}_{d(N)} & {\left[e^{\left.-\Lambda_{N}^{c} \int_{0}^{t} \mathbf{1}_{\left(\left|X_{u}^{N}\right|=\frac{N-1}{N}\right.}\right) d u-\Lambda_{N-1}^{c} \int_{0}^{t} \mathbf{1}_{\left(\left|X_{u}^{N}\right|=\frac{N-2}{N}\right)} d u}\right.} \\
& \left.\times f\left(\left|X_{t}^{N}\right|+\frac{1}{N}\right)\left(\mathbf{1}_{\left(\tau_{N}^{\alpha} \leq t<\tau_{N}^{0}+\lambda\right)}+\mathbf{1}_{\left(t \geq \tau_{N}^{0}+\lambda\right)}\right)\right],
\end{aligned}
$$


where $\tau_{N}^{0}=\tau_{(N-1) / N}^{(1)} \wedge \tau_{-(N-1) / N}^{(1)}$ and $\lambda \in(0, t)$. Consequently,

$$
\lim _{N^{\prime}} \mathbb{E}_{d\left(N^{\prime}\right)}\left[f\left(\left|X_{t}^{N^{\prime}}\right|+\frac{1}{N^{\prime}}\right) \mathbf{1}_{\left(t<\tau_{N^{\prime}}^{\alpha}\right)}\right]=\mathbb{E}^{\mathbb{Q}_{u}}[f(|x(t)|)]
$$

and $\mathbb{E}^{\mathbb{Q}_{u}}\left[f(|x(t)|) \mathbf{1}_{\left(\tau_{1} \leq t\right)}\right]=0$ by the Portmanteau theorem (see [12]).

The following lemma represents the loss of energy from the propagating modes produced by the coupling between the propagating and the radiating modes. Moreover, this lemma gives us the absorbing condition at the boundary $u=1$ in Theorem 6.2 , and then the dissipation behavior in Theorem 6.3.

Lemma A.10. $\lim _{N^{\prime}} r\left(N^{\prime}\right)=0$.

A full proof of this lemma is given in [12]. However, in the following sketch of proof we give the main ideas.

Proof.

$$
\begin{aligned}
& \left|r\left(N^{\prime}\right)\right| \leq\|f\|_{\infty}\left(\mathbb{E}_{d\left(N^{\prime}\right)}\left[e^{\left.-\Lambda_{N^{\prime}}^{c} \int_{0}^{t} \mathbf{1}_{\left(\left|X_{u}^{N^{\prime}}\right|=\frac{N^{\prime}-1}{N^{\prime}}\right)}\right)^{d u}} \mathbf{1}_{\left(t \geq \tau_{N^{\prime}}^{0}+\lambda\right)}\right]\right. \\
& \left.+\mathbb{P}_{d\left(N^{\prime}\right)}\left(\left|X_{t}^{N^{\prime}}\right|+\frac{1}{N^{\prime}} \in \operatorname{supp}(f), \tau_{N^{\prime}}^{\alpha} \leq t<\tau_{N^{\prime}}^{0}+\lambda\right)\right) .
\end{aligned}
$$

The first term on the right of the previous inequality goes to 0 as $N \rightarrow+\infty$, because the jump process $X^{N}$ can visit $\pm(N-1) / N$ sufficiently often during a time interval of order 1 , and $\Lambda_{N}^{c} \geq K N^{3 / 2}$. Next, for $\lambda \ll 1$, after having visited $\left(N-\left[N^{\alpha}\right]\right) / N$ (resp., $\left.-\left(N-\left[N^{\alpha}\right]\right) / N\right)$, the jump process reaches $(N-1) / N$ (resp., $\left.-(N-1) / N\right)$ after a very small time. Then, after having visited $\pm\left(N-\left[N^{\alpha}\right]\right) / N, X^{N}$ has a very little time, of order $\lambda$, to reach $\operatorname{supp}(f)$. Consequently, the second term on the right of the previous inequality goes also to 0 as $N \rightarrow+\infty$ and $\lambda \rightarrow 0$.

As a result, $\left(\mathcal{T}_{f}^{l(N)}(\omega, t)\right)_{N}$ is a bounded sequence and all the subsequence limits of $\left(\mathcal{T}_{f}^{l(N)}(\omega, t)\right)_{N}$ are equal to $\mathbb{E}^{\overline{\mathbb{P}}_{u}}\left[f(|x(t)|) \mathbf{1}_{\left(t<\tau_{1}\right)}\right]$. Consequently,

$$
\lim _{N} \mathcal{T}_{f}^{l(N)}(\omega, t)=\mathbb{E}^{\overline{\mathbb{P}}_{u}}\left[f(|x(t)|) \mathbf{1}_{\left(t<\tau_{1}\right)}\right] .
$$

Now, we have to show that this equality holds even for a sequence $(l(N))_{N}$ such that $l(N) / N \rightarrow u=1$, i.e $\lim _{N} \mathcal{T}_{f}^{l(N)}(\omega, t)=0$. To do this, we write for $\lambda \in(0, t)$,

$$
\begin{aligned}
& \mathcal{T}_{f}^{l(N)}(\omega, t) \leq\|f\|_{\infty}\left(\mathbb{P}_{d(N)}\left(t<\tau_{(N-1) / N)}^{(1)}+\lambda\right)\right.
\end{aligned}
$$

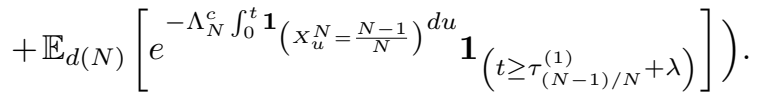

The second term on the right in the previous inequality goes to 0 for the same reasons as in the proof of Lemma A.10. Moreover, we have

$$
\mathbb{P}_{d(N)}\left(t<\tau_{(N-1) / N)}^{(1)}+\lambda\right) \leq \frac{1}{t-\lambda} \mathbb{E}_{d(N)}\left[\tau_{(N-1) / N)}^{(1)}\right] \leq K\left(1-\frac{l(N)}{N}\right) .
$$

Consequently, we have $\forall u \in[0,1]$ and $\forall(l(N))_{N}$ such that $l(N) / N \rightarrow v$,

$$
\lim _{N} \mathcal{T}_{f}^{l(N)}(\omega, t)=\mathbb{E}^{\overline{\mathbb{P}}_{v}}\left[f(|x(t)|) \mathbf{1}_{\left(t<\tau_{1}\right)}\right],
$$


where this limit satisfies the required conditions.

Acknowledgement. I wish to thank my Ph.D. supervisor Josselin Garnier for his suggestions and his support.

\section{REFERENCES}

[1] R.J. Adler, The Geometry of Random Fields, Wiley, London, 1981.

[2] R.J. Adler and J. Taylor, Random Fields and Geometry, Springer, New York, 2007.

[3] P. Billingsley, Convergence of Probability Measure, $2^{\text {nd }}$ ed., Wiley InterScience, 1999.

[4] R. Carmona and J.-P. Fouque, Diffusion-approximation for the advection-diffusion of a passive scalar by a space-time gaussian velocity field, Seminar on Stochastic Analysis, Random Fields and Applications, E. Bolthausen, M. Dozzi and F. Russo, eds., Birkhauser, Basel, 37-50, 1994.

[5] M.D. Donsker and S.R.S. Varadhan, Asymptotic evaluation of certain Markov process expectations for large time, I-IV, Commun. Pure Appl. Math, 28, 1-47, 279-301, 1975; 29, 368-461, 1979; 36, 183-212, 1983.

[6] J.P. Fouque, La convergence en loi pour les processus à valeur dans un espace nucléaire, Ann. Inst. Henri Poincaré, 20, 225-245, 1984.

[7] J.P. Fouque, J. Garnier, G. Papanicolaou, and K. Sølna, Wave Propagation and Time Reversal in Randomly Layered Media, Springer, New York, 2007.

[8] J. Garnier, The role of evanescent modes in randomly perturbed single-mode waveguides, Discrete and Continuous Dynamical Systems-Series B, 8, 455-472, 2007.

[9] J. Garnier and G. Papanicolaou, Pulse propagation and time reversal in random waveguides, SIAM J. Appl. Math., 67, 1718-1739, 2007.

[10] J. Garnier and K. Sølna, Effective transport equations and enhanced backscattering in random waveguides, SIAM J. Appl. Math., 68, 1574-1599, 2008.

[11] C. Gomez, Time-reversal superresolution in random waveguides, SIAM Multiscale Model. Simul., 7, 1348-1386, 2009.

[12] C. Gomez, Wave propagation in shallow-water acoustic random waveguides, arxiv:0911.5646v1 [math PR], preprint, 2009.

[13] F.B. Jensen, W.A. Kuperman, M.B. Porter and H. Schmidt, Computational Ocean Acoustics, AIP Press, Springer, New York, 2000.

[14] G. Kallianpur and J. Xiong, Stochastic Differential Equations in Infinite Dimensional Spaces, IMS Lecture notes-monograph series, 1995.

[15] W. Kohler and G. Papanicolaou, Wave Propagation in Randomly Inhomogeneous Ocean, Lecture Notes in Physics, J.B. Keller and J.S. Papadakis, eds., Wave Propagation and Underwater Acoustics, Springer-Verlag, Berlin, 70, 1977.

[16] R.Z. Khasminskii, A limit theorem for solutions of differential equations with random right hand side, Theory Probab. Appl., 11, 390-406, 1966.

[17] W.A. Kuperman and J.F. Lynch, Shallow-Water acoustics, Phys. Today 57, October, 55, 55-61, 2004.

[18] H.J. Kushner, Approximation and Weak Convergence Methods for Random Processes, MIT press, Cambridge, 1984.

[19] R. Magnanini and F. Santosa, Wave propagation in a 2-D optical waveguide, SIAM J. Appl. Math., 61, 1237-1252, 2000.

[20] D. Marcuse, Theory of Dielectric Optical Waveguides, $2^{\text {nd }}$ ed., Academic press, New York, 1991.

[21] R.H. Mellen, D.G. Browning and J.M. Ross, Attenuation in randomly inhomogeneous sound chanels, J. Acoust. Soc. Am., 56(3), 80-82, 1974.

[22] M. Metivier, Stochastic Partial Differential Equations in Infinite Dimensional Spaces, Scuola normale superiore, Pisa, 1988.

[23] I. Mitoma, On the sample continuity of $\mathcal{S}^{\prime}$-processes, J. Math. Soc. Japan, 35, 629-636, 1983.

[24] G. Papanicolaou and W. Kohler, Asymptotic theory of mixing stochastic ordinary differential equations, Commun. Pure Appl. Math., 27, 641-668, 1974.

[25] C.I. Pekeris, Theory of propagation of explosive sound in shallow water, Propagation of Sound in the Ocean, Geological Society of America, Memoir, 27, 1-117, 1948.

[26] E. Perrey-Debain and I.D. Abrahams, A diffusion analysis approach to TE mode propagation in randomly perturbed optical waveguides, SIAM J. Appl. Math., 68, 523-543, 2007.

[27] H.E. Rowe, Electromagnetic Propagation in Multi-mode Random media, Wiley, New York, 1999. 
[28] H. Schaefer, Topological Vector Spaces, Springer-Verlag, New York, 1971.

[29] D.W. Stroock and S.R.S. Varadhan, Multidimensional Diffusion Processes, Springer-Verlag, Berlin, 1979.

[30] C. Wilcox, Spectral analysis of the Pekeris operator in the theory of acoustic wave propagation in shallow water, Arch. Rational Mech. Anal., 60(3), 259-300, 1976.

[31] C. Wilcox, Transient electromagnetic wave propagation in a dielectric waveguide, in Symposia Mathematica, vol. XVIII (Convegno sulla Teoria Matematica dell'Elettromagnetismo, INDAM, Rome, 1974), Academic Press, London, 239-277, 1976.

[32] M. Yor, Existence et unicité de diffusion à valeurs dans un espace de Hilbert, Ann. Inst. Henri Poincaré, 10, 55-88, 1974. 